\title{
Investigations of round vertical turbulent buoyant jets
}

\author{
By PANOS N. PAPANICOLAOU† AND E. JOHN LIST $\ddagger$ \\ †University of California San Diego, Department of Ames, B-010, La Jolla, CA 92093, USA \\ $\ddagger$ W. M. Keck Laboratory of Hydraulies and Water Resources California Institute of \\ Technology Pasadena, CA 91125, USA
}

(Received 3 April 1987 and in revised form 16 February 1988)

The axial and radial velocity components $w$ and $u$, and the concentration $c$ of a Rhodamine 6G dye were measured simultaneously in a turbulent buoyant jet, using laser-Doppler anemometry combined with a recently developed laser-inducedfluorescence concentration measurement technique. These non-intrusive techniques enable measurements in a region of plume motion where conventional probe-based techniques have had difficulties. The results of the study show that the asymptotic decay laws for velocity and concentration of a tracer transported by the flow are verified experimentally in both jets and plumes. The momentum and volume fluxes and the mean dilution factor are determined in dimensionless form as a function of the normalized distance from the flow source. Contradictory results from earlier experimental plume investigations concerning the decay laws of $w$ and $c$ and the plume width ratio $b_{c} / b_{w}$ are discussed. The turbulence properties and the transition from momentum-driven jets to buoyancy-driven plumes are presented. The turbulence is found to scale with the mean flow as predicted by dimensional analysis and self-similarity. Buoyancy-produced turbulence is found to transport twice as much tracer as jet turbulence. Although velocity statistics in jets and plumes are found to be highly self-similar there is a strong disparity in the distribution of tracer concentration in the two flows. This occurs in the time-average mean flows as well as the r.m.s. turbulent quantities. Instantaneous concentration fluctuations are found to exceed time averages by as much as a factor of 3 . The experimental results should provide a reasonable basis for validation of computer models of axisymmetric plumes.

\section{Introduction}

Turbulent buoyant jets are an integral part of many waste disposal systems, including diffuser outfalls, smoke stacks, cooling towers and fires. They also occur quite naturally in association with volcanos and the newly observed deep-sea 'black smokers'. Understanding the mechanics of such flows is a key element in the optimal design of waste discharge facilities and much is already known and successfully applied. However, the research literature on buoyant jets is remarkably meagre in relation to that describing studies of homogeneous fluid jets. In this paper we describe the results of a detailed laboratory study of the motion of jets whose mechanics are influenced strongly by a difference in density between the ambient fluid and the discharged fluid. The results will be of interest and concern to hydraulic engineers who design discharge facilities, and oceanographers interested in deep-sea vents. 
Prior work on turbulent buoyant jets has been reviewed in depth in two recent articles (List $1982 a, b$ ). The main conclusion resulting from those reviews was that there were some remaining issues that further experimental work would help to resolve. In particular, it was concluded that the appropriateness of the commonly employed self-similar hypotheses used to describe jet and plume motion should be further re-evaluated. In addition, it was felt that experimental tracer and momentum fluxes would help establish the relative importance of the various mechanisms responsible for transfer processes in buoyancy-driven motions. (The practical application of buoyant-jet theory relies significantly upon these two fundamental issues.)

The two key assumptions upon which design theory is based are that a jet's momentum flux is preserved with no modification of fluid motion by density variations, whereas in a plume the flux of density-deficiency is preserved. In plumes momentum flux is continuously modified by the application of buoyancy forces resulting from this density anomaly. These two basic assumptions enable a complete theory to be developed to describe the evolution of a buoyant jet, including lateral growth rate, rate of entrainment of ambient fluid, rate of decay of mean axial velocity, rate of dilution of tracer material and so forth (Fischer et al. 1979). However, the theory is not so complete as to provide values of fundamental constants inherent in the dimensionless relationships developed. It is well known that at the present time no such theory of turbulent flows exists. However, it is apparent that direct numerical solution of the Navier-Stokes equations is coming close to accomplishing this for some flow configurations. Thus, in the absence of a complete turbulence theory or access to direct numerical solutions, experiment provides the only method for determining adequate parameters for design. Apart from these design motivations for understanding the development of mean quantities within turbulent buoyant jets there are often more fundamental questions that remain unanswered. It is clear, for example, that the energetics of the turbulence must undergo fundamental changes as the transition from jets to plumes occurs. At the present time experiments are the only recourse in establishing exactly how this transition occurs.

It is somewhat surprising, given the importance of convective phenomena in geophysical fluid dynamies, that the production of turbulence in isolated convective flows has not received much experimental attention. Kotsovinos (1977) in a pioneering study observed turbulence in plane turbulent buoyant jets using laserDoppler velocimetry combined with tracer concentration measurements based on microbead thermistor measurement of temperature fluctuations in jets and plumes. The instrumentation employed by Kotsovinos was crude by modern standards and the results he obtained were remarkable in that a first attempt was made to measure turbulent fluxes directly. He found mean turbulent fluxes as high as $10 \%$ of the mean advective flux in jets and as high as $40 \%$ in plane plumes. Ramaprian \& Chandrasekhara (13) have since repeated Kotsovinos' experiment and found $15 \%$ turbulent fluxes in plane plumes. This latter study also employed thermistor-based temperature measurements. Nakagome \& Hirata (1976) and George, Alpert \& Tamanini (1977) also attempted to measure turbulence properties in round buoyant jets using hot-wire equipment. They both determined lower turbulent flux contributions than Kotsovinos for plane plumes. However, in these latter studies it was not at all clear that the experimental plumes were fully developed into selfsimilar flows.

The experimental study described in this paper used new experimental techniques 
based on combined application of laser-Doppler anemometry and laser-inducedfluorescence concentration measurement. These non-intrusive optically based measurement systems have enabled turbulence-flux measurements at a single point in space. Specific details of the experimental configuration and resolution, and data collection and analysis methods are given in the Appendix.

The basic objective of the studies described here is an elucidation of the mechanics that produce turbulent mixing in jets and plumes. Jets have received a significant level of prior study and this work was reviewed recently by one of the authors (List $1982 a, b)$. Subsequent work by others (Hussain \& Clark 1981) has shown in great detail the importance of large-scale flow structure within jets. In plumes, however, there is both the production of turbulence by shear stress/mean shear interactions as in jets and, in addition, direct transfer of potential energy to turbulent kinetic energy through buoyant work done. Unravelling the entire distribution of energy within such flows is complex enough in itself and will be the subject of another paper. Here we present the description of the phenomena as observed in the detailed simultaneous measurements of velocity and tracer concentration.

\section{Experimental equipment and techniques}

The experimental investigations studied round turbulent vertical buoyant jets over a wide range of initial flux Richardson number, which is a dimensionless parameter that defines the degree of jet-like or plume-like character of the initial flow. It is defined as

$$
R_{0}=\frac{Q B^{\frac{1}{2}}}{M^{\frac{5}{4}}}
$$

where $Q$ is the initial volume flux, $B$ is the initial specific buoyancy flux and $M$ is the initial specific momentum flux. In other words

$$
B=g \frac{(\Delta \rho)_{0}}{\rho_{\mathrm{a}}} Q
$$

is the flux of initial density deficiency $(\Delta \rho)_{0}$ of the source fluid with $g$ the gravitational acceleration. The specific momentum flux is given by

$$
M=Q W,
$$

where $W$ is the source mean velocity. The initial Richardson number $R_{0}$ varies from very small for jet-like flows to order unity for plume-like flows.

Experiments were carried out in a $2.54 \mathrm{~cm}$ thick glass-walled tank with a crosssection $1.15 \times 1.15 \mathrm{~m}$ and $3.35 \mathrm{~m}$ deep. The buoyant jets studied, which were aligned with the vertical axis of this tank, issued from an orifice $0.752 .0 \mathrm{~cm}$ in diameter supplied by a constant-head tank. Vertical $(w)$ and horizontal (radial $u$ ) fluid velocities and Rhodamine 6G dye concentrations $(c)$ were measured simultaneously at the same point of flow by a laser-Doppler velocimeter (LDV) combined with a laser-induced-fluorescence (LIF) system. The tank was equipped with an instrument carriage that supported the LDV and LIF concentration measuring system. The carriage was able to move both vertically and horizontally so that measurements of velocity and concentration could be obtained at various axial and radial locations in vertical planes containing the jet axis. The jet was located either deep in the tank and pointed vertically upwards, or at the top of the tank and pointed down (figure 1). In the first case the jet fluid was less dense than the ambient fluid and vice versa in the 


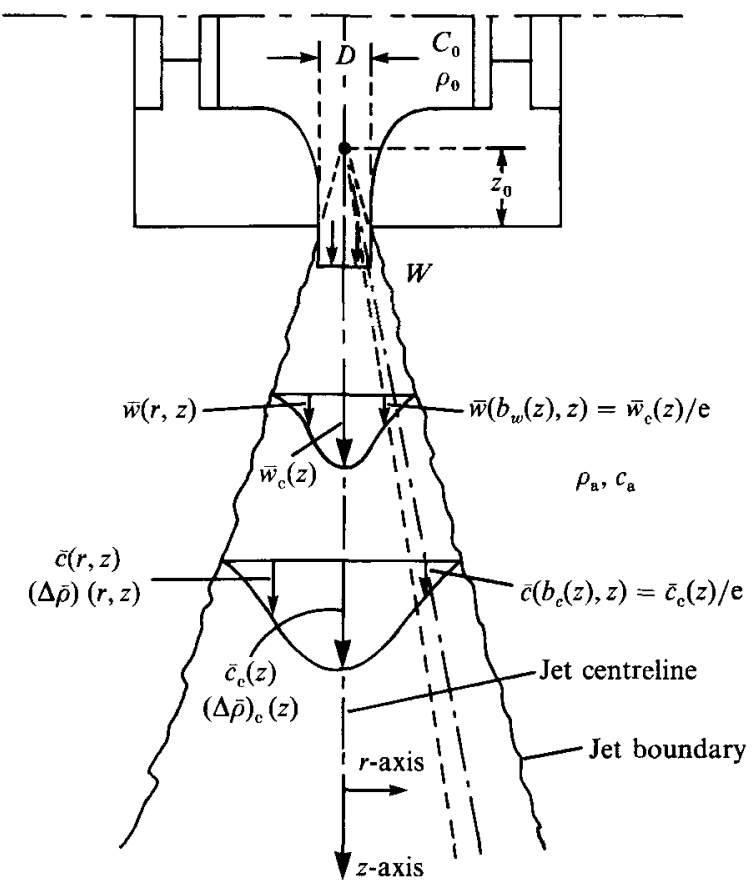

Figure 1. Flow configuration : geometry of a buoyant jet.

second case. Sodium chloride-water solutions were used to obtain the desired initial density difference between the jet and the ambient fluid. The cxperimental set-up, techniques and procedures applied are described in detail in the Appendix. It is important to note here that the use of LIF to measure absolute concentrations for the purpose of mass flux measurement was subject to error, and the difficulties encountered in the simultaneous use of laser-Doppler anemometry and LIF will be discussed in connection with the presentation of the experimental measurements of mass flux.

The direct objective of the study was the measurement of fluid velocity and tracer concentration in both jet and plume flow regimes and the transition region. According to the preliminary thermistor probe measurements described in Papanicolaou \& List (1987), the dimensionless distance $z / l_{M}$ from the flow source has to vary from very small in jets to over 20 in plumes to obtain asymptotic flows, where

$$
l_{M}=\frac{M^{\frac{3}{4}}}{B^{\frac{1}{2}}}
$$

is the characteristic lengthscale describing the relative importance of momentum and buoyancy fluxes (Fischer et al. 1979). The important parameters in the selection of the distance $z$ for the experimental cross-section under consideration are thus the specific momentum flux $M$ and specific buoyancy flux $B$, the latter being controlled by the jet tracer concentration $C_{0}$ and the density difference between the jet and the ambient fluid. Three series of experiments were performed as defined in tables 1 and 2. The EXP-series of experiments involved simultaneous $w, u$ and $c$ measurements at various distances $z / l_{M}$ from the origin. The VEL and PAP series of experiments consist of only axial and radial velocity measurements in jets $\left(z / l_{M} \ll 1\right)$. 


\begin{tabular}{|c|c|c|c|c|c|c|c|c|c|}
\hline & $\begin{array}{c}z \\
\mathrm{~cm}\end{array}$ & $\begin{array}{c}D \\
\mathrm{~cm}\end{array}$ & $\begin{array}{c}W \\
\mathrm{~cm} / \mathrm{s}\end{array}$ & $\begin{array}{c}\bar{w}_{\mathrm{c}} \\
\mathrm{cm} / \mathrm{s}\end{array}$ & $\begin{array}{l}\left(\overline{w_{\mathrm{c}}^{\prime 2}}\right)^{\frac{1}{2}} \\
\mathrm{~cm} / \mathrm{s}\end{array}$ & $\begin{array}{l}\left(\overline{u_{\mathrm{c}}^{\prime 2}}\right)^{\frac{1}{2}} \\
\mathrm{~cm} / \mathrm{s}\end{array}$ & $\begin{array}{c}\frac{(\Delta \rho)_{0}}{\rho_{\mathrm{a}}} g \\
\mathrm{~cm} / \mathrm{s}^{2}\end{array}$ & $\begin{array}{c}l_{M} \\
\mathrm{~cm}\end{array}$ & $\begin{array}{c}b_{w} \\
\mathrm{~cm}\end{array}$ \\
\hline VELO5 & 67.100 & 0.750 & 79.210 & 5.800 & 1.517 & 0.929 & 0.085 & 222.130 & 7.440 \\
\hline VEL07 & 50.400 & 0.750 & 66.613 & 7.000 & 1.675 & 1.040 & 0.131 & 150.200 & 5.712 \\
\hline VEL10 & 64.900 & 0.750 & 80.781 & 6.131 & 1.487 & 0.934 & 0.197 & 148.534 & 6.468 \\
\hline VEL11 & 77.300 & 0.750 & 95.736 & 5.612 & 1.487 & 0.963 & 0.287 & 145.690 & 8.006 \\
\hline VEL13 & 33.500 & 0.750 & 55.590 & 7.900 & 1.780 & 1.165 & 0.263 & 88.300 & 3.987 \\
\hline VEL15 & 60.500 & 0.750 & 87.080 & 7.250 & 1.621 & 1.159 & 0.263 & 138.320 & 6.667 \\
\hline VEL17 & 82.500 & 0.750 & 111.085 & 6.750 & 1.768 & 1.141 & 0.281 & 170.820 & 8.607 \\
\hline VEL19 & 63.750 & 0.750 & 94.950 & 7.500 & 1.700 & 1.159 & 0.351 & 130.660 & 6.324 \\
\hline VEL21 & 45.000 & 0.750 & 63.464 & 7.320 & 1.600 & 1.000 & 0.260 & 101.380 & 4.657 \\
\hline VEL23 & 26.250 & 0.750 & 40.638 & 7.100 & 1.597 & 0.843 & 0.306 & 59.920 & 2.820 \\
\hline PAP12 & 48.750 & 0.750 & 61.890 & 6.143 & 1.492 & 0.905 & 0.192 & 115.260 & 4.530 \\
\hline PAP13 & 63.750 & 0.750 & 110.690 & 7.850 & 1.718 & 1.115 & 0.279 & 170.810 & 6.711 \\
\hline PAP16 & 18.250 & 0.750 & 31.980 & 6.900 & 1.762 & 1.021 & 0.110 & 78.570 & 2.370 \\
\hline PAP17 & 37.500 & 0.750 & 70.550 & 8.600 & 2.351 & 1.437 & 0.088 & 194.440 & 4.124 \\
\hline PAP20 & 75.000 & 0.750 & 142.180 & 8.800 & 2.133 & 1.527 & 0.043 & 557.300 & 7.956 \\
\hline PAP21 & 52.500 & 0.750 & 94.950 & 8.200 & 1.913 & 1.421 & 0.043 & 372.220 & 5.774 \\
\hline PAP30 & 66.000 & 0.750 & 140.600 & 11.000 & 2.517 & 1.581 & 0.150 & 296.075 & 6.788 \\
\hline PAP32 & 38.250 & 0.750 & 79.206 & 10.500 & 2.232 & 1.593 & 0.150 & 166.790 & 3.922 \\
\hline PAP31 & 51.600 & 0.750 & 98.880 & 9.147 & 2.275 & 1.521 & & & \\
\hline PAP34 & 79.350 & 0.750 & 140.600 & 8.468 & 1.999 & 1.312 & & & \\
\hline PAP34 & 70.650 & 0.750 & 140.600 & 9.936 & 2.396 & 1.476 & & & \\
\hline PAP34 & 60.950 & 0.750 & 140.600 & 11.321 & 2.820 & 1.733 & & & \\
\hline PAP34 & 49.350 & 0.750 & 63.460 & 5.832 & 1.559 & 0.857 & & & \\
\hline PAP34 & 40.350 & 0.750 & 63.460 & 7.711 & 1.664 & 1.038 & & & \\
\hline PAP34 & 30.850 & 0.750 & 47.720 & 7.273 & 1.525 & 0.958 & & & \\
\hline PAP34 & 26.250 & 0.750 & 31.980 & 5.361 & 1.153 & 0.713 & & & \\
\hline PAP34 & 21.000 & 0.750 & 24.110 & 4.960 & 0.999 & 0.646 & & & \\
\hline PAP34 & 75.000 & 0.750 & 126.430 & 8.169 & 1.878 & 1.288 & & & \\
\hline PAP34 & 54.600 & 0.750 & 94.950 & 8.754 & 1.945 & 1.196 & & & \\
\hline
\end{tabular}

TABLE 1. Initial and measured flow parameters for the axial and radial velocity measurements in jets $\left(z / l_{M}<1\right.$, VEL and PAP series $)$.

The initial parameters of the flow in SI units listed in tables 1 and 2 are the following: $z(\mathrm{~cm})$ is the axial distance from the origin of the jet transverse section under consideration, $D(\mathrm{~cm})$ is the jet diameter, $W(\mathrm{~cm} / \mathrm{s})$ is the cross-sectionally averaged jet exit velocity at the nozzle, $C_{0}$ (p.p.b.) is the excess (above the ambient) tracer concentration (Rhodamine $6 \mathrm{G}$ dye) at the jet orifice, $g_{0}^{\prime}=\left[(\Delta \rho)_{0} / \rho_{\mathrm{a}}\right] g\left(\mathrm{~cm} / \mathrm{s}^{2}\right)$ is the reduced gravitational acceleration at the jet exit, and $l_{M}(\mathrm{~cm})$ is the characteristic jet momentum lengthscale as defined previously. The measured quantities at a jet cross-section at a distance $z$ from the origin are: $\bar{w}_{\mathrm{c}}(\mathrm{cm} / \mathrm{s})$ the mean centreline velocity, $\bar{c}_{\mathrm{c}}$ (p.p.b.) the mean excess concentration of Rhodamine $6 \mathrm{G}$ dye at the axis, and $b_{w}(\mathrm{~cm})$ and $b_{c}(\mathrm{~cm})$ the axial mean velocity and concentration lengthscales defined by the point on the profile at which the value is $1 / \mathrm{e}$ times the maximum value.

Data were collected electronically using laboratory developed hardware and software described in detail in Papanicolaou (1984). The data sampling rate varied from $25 \mathrm{~Hz}$ in plumes to $50 \mathrm{~Hz}$ in jets for a sampling period that exceeded $120 \mathrm{~s}$. Statistical analysis of the data was based on the discrete velocity components and the concentration, and the $1 / \mathrm{e}$ velocity and concentration widths were calculated 


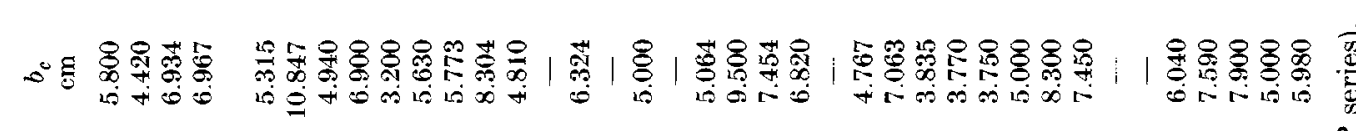

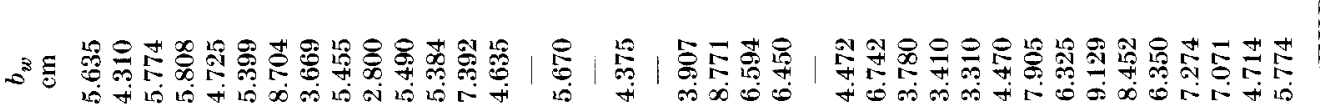

|

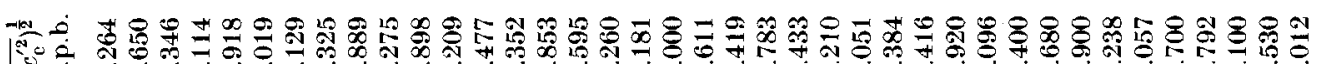

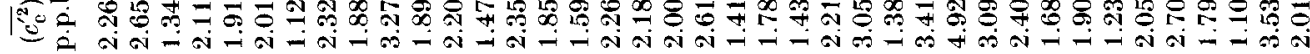

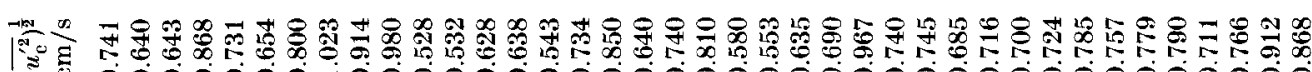

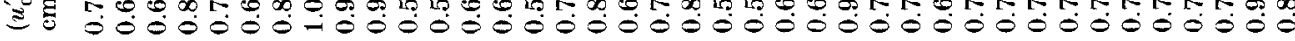

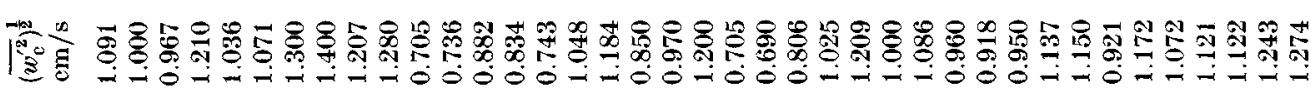

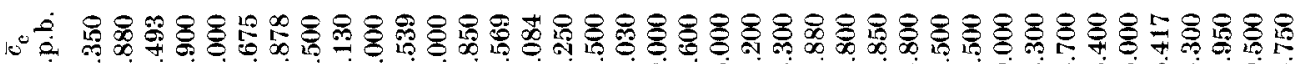

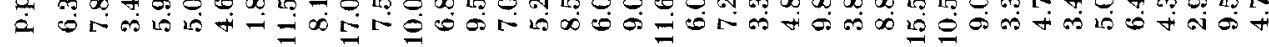

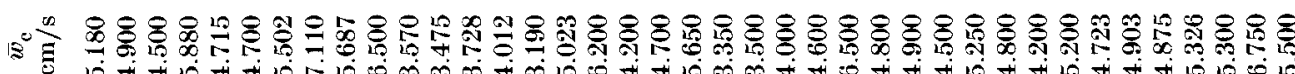

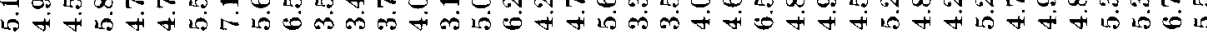

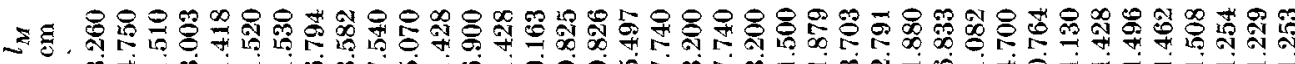

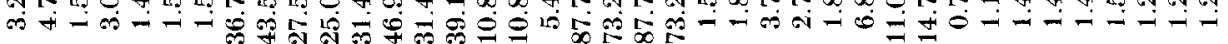

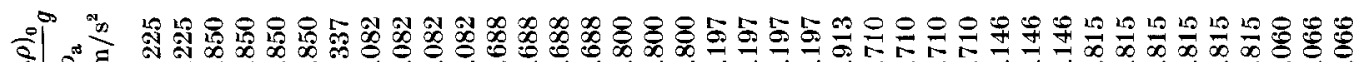
ये|

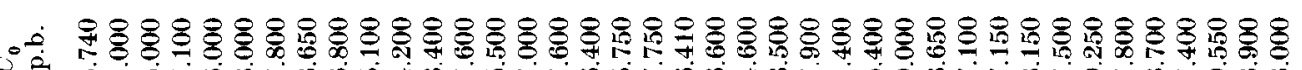

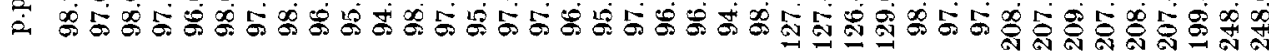

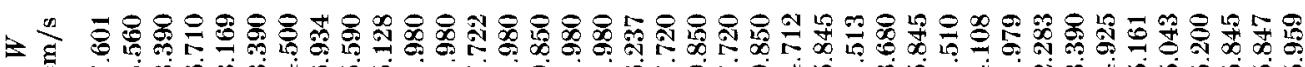

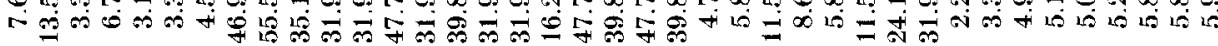

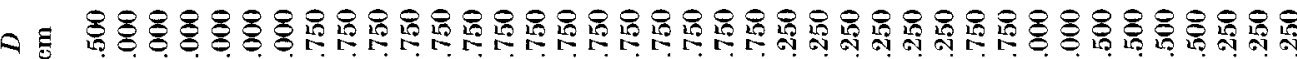
-

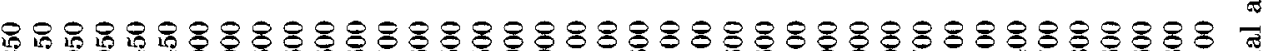

n

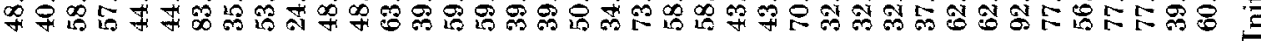

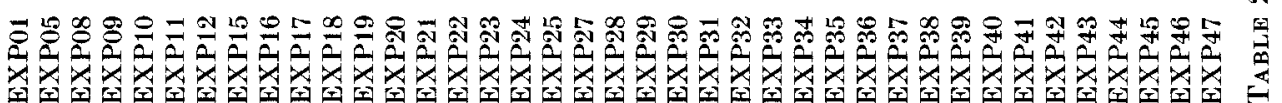




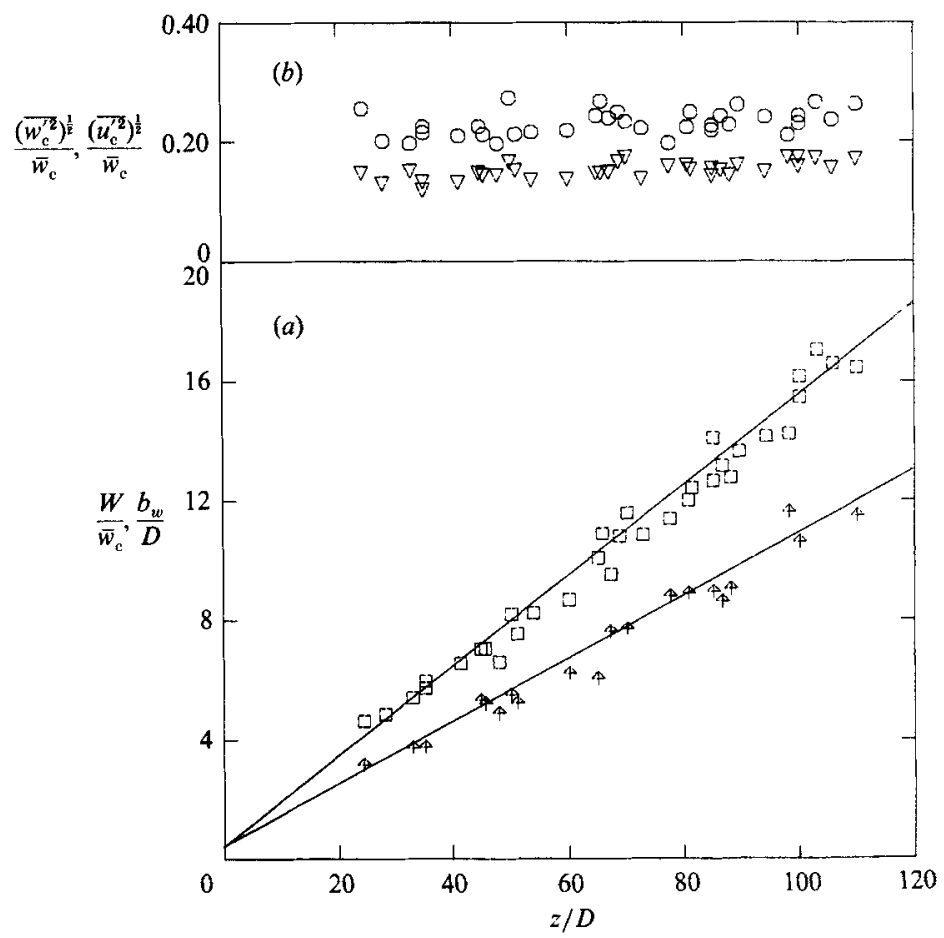

Figure 2. Non-dimensional $(a) \square$, mean velocity; and $\uparrow$, jet width; and $(b)$ r.m.s. O. axial; and $\nabla$, radial velocity along the jet axis, versus $z / D$.

from Gaussian curves fitted to the time-averaged velocity and concentration crosssectional profiles. The range of initial Reynolds numbers at the nozzle varied from 600 in plumes to about 11000 in jets.

\section{Mean-flow experimental results}

The fully developed self-similar momentum-jet flow regime has been examined thoroughly in a number of prior experimental studies and there exist consistently good experimental data that describe completely the momentum-jet flow field (List $1982 a$ ). However, as previously noted very few of these studies have investigated the transition from jets to plumes. In what follows we briefly present the mean-flow results of pure jets, jets in transition, and plumes. The jet results presented do not indicate anything previously not known. However, they are important in validating the experimental methods in their extension to buoyant flows.

The time-averaged normalized longitudinal velocity along the jet axis is plotted in figure $2(a)$ versus the dimensionless distance $z / D$ from its origin. These data points correspond to the VEL and PAP series of experiments and those experiments from the EXP series for which $z / l_{M}<1$. A least-square linear fit to the data points is

$$
\frac{W}{\bar{w}_{\mathrm{c}}}=0.149\left(\frac{z}{D}+2.56\right)
$$

thus defining a virtual origin for the mean centreline velocity at 2.56 diameters upstream from the jet nozzle. The data points for $z / D<50$ show a tendency to shift 
the virtual origin further upstream. This is in agreement with Wygnanski \& Fiedler's (1969) observation that the slope of the data fit is smaller in the initial 50 jet diameters. In figure $2(b)$ the normalized intensity of turbulence of the axial and radial velocities take constant values beyond 50 jet diameters equal to $\left(\overline{w_{\mathrm{c}}^{\prime 2}}\right)^{\frac{1}{2}} / \bar{w}_{\mathrm{c}}=$ 0.25 and $\left(\overline{u_{\mathrm{c}}^{\prime 2}}\right)^{\frac{1}{2}} / \bar{w}_{\mathrm{c}}=0.17$. Wygnanski \& Fiedler reported that the axial and radial turbulent velocities became constant and equal to 0.28 and 0.25 after about 40 and 70 diameters downstream respectively, which are higher than for the present experiments. This may well be a result of the normalization with $\bar{w}_{\mathrm{c}}$, which was measured to be higher than that reported by the above investigators. One possible explanation for the higher observed $\bar{w}_{\mathrm{c}}$ may be the relatively low jet Reynolds numbers (2500-11000) of the present investigation compared with about $10^{5}$ in the Wygnanski \& Fiedler (1969) study (cf. turbulent pipe flow where higher Reynolds number results in lower mean velocities at the pipe axis). Most jet investigators have reported the axial turbulence intensity to be higher than the radial and tangential turbulence intensity, except Antonia, Prabhu \& Stevenson (1975) and Gibson (1963), who measured the turbulence intensity to be the same, 0.30 , in all three directions, implying that the turbulence is isotropic near the jet axis. A Gaussian fit to the transverse mean velocity profiles of the form $\bar{w}=\bar{w}_{\mathrm{c}} \exp \left(-k r^{2}\right)$ leads to a definition of the jet width $b_{w}$ as the $1 /$ e-point of the profile. It is found that

$$
\frac{b_{w}}{D}=0.104\left(\frac{z}{D}+2.58\right)
$$

which is also plotted on figure 2 and indicates that the virtual origin for the jet width is at the same location as that for the mean axial velocity. The coefficient of 0.104 is in agreement with the mean value of 0.107 from different investigations given by Fischer et al. (1979). The scatter of the data points in figure $2(a, b)$ is attributed to the fact that all measurements were performed at different jet initial conditions except for the jet diameter, which was kept constant. A slight inclination of the jet could also bias the measurement by offsetting the measurement cross-section from the jet axis, resulting in narrower mean velocity profiles and lower centreline velocities. The experiments indicate a value of $k$ in the velocity profile of 93 for $z / D>50$ and 80 for $z / D<50$.

The reciprocal of the time-averaged centreline excess (above the ambient) concentration, normalized by the initial jet excess concentration $C_{0} / \bar{c}_{\mathbf{c}}$, is plotted against $z / D$ in figure 3. A linear fit to the data pairs is $C_{0} / \bar{c}_{\mathrm{c}}=0.157(z / D+4.35)$ implying that a virtual origin for the mean centreline concentration is located 4.35 jet diameters upstream from the nozzle. The normalized centreline r.m.s. concentration $\left(\overline{c_{\mathrm{e}}^{\prime 2}} \frac{1}{2} / \overline{c_{\mathrm{c}}}\right.$ is plotted as a function of $z / D$ in the same figure and takes a constant value of 0.22 for $z / D>40$. The mean centreline concentration reported here is higher than that reported by Becker, Hottel \& Williams (1967), Kiser (1963), Forstall \& Gaylord (1955), Grandmaison, Rathgeber \& Becker, (1977) and Birch et al. (1978), who have reported coefficients varying from 0.185 to 0.250 . This result is important since it indicates a potential problem with the LIF method of concentration measurement. It will be discussed in more detail in connection with the turbulence data presentation. However, the turbulence intensity agrees with that reported by the above authors. The dimensionless concentration 1/e-width $b_{c}(z) / D$, (obtained by fitting a Gaussian profile to the experimental data), plotted as a function of $z / D$ gives

$$
\frac{b_{c}(z)}{D}=0.139\left(\frac{z}{D}-2.51\right)
$$




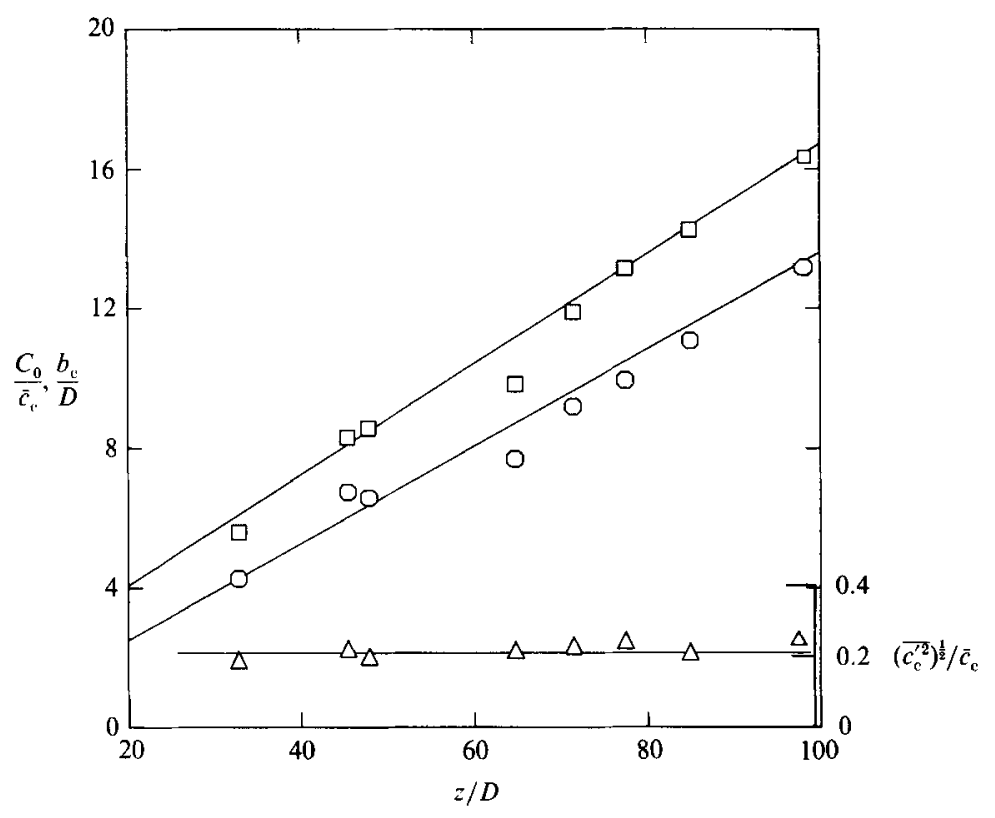

Figure 3. Non dimensional concentration distribution along the jet axis: $\square$, mean concentration ; $\triangle$, r.m.s. concentration; and $O, 1 /$ e concentration width.

implying a virtual origin 2.5 diameters downstream from the jet nozzle. Different investigators have reported values varying from 0.101 to 0.140 for the constant of proportionality. Fischer et al. (1979) suggest an average value of 0.127 for a jet. A least-square Gaussian fit to the profile data is $\bar{c} / \bar{c}_{\mathrm{c}}=\exp \left[-52(r / z)^{2}\right]$.

In a jet or a plume the specific momentum flux (momentum flux per unit mass) at a distance $z$ from the origin is

$$
m(z)=\int_{0}^{b(z)}\left(\bar{w}^{2}+\overline{w^{2}}\right) 2 \pi r \mathrm{~d} r .
$$

Following List $(\mathbf{1 9 8 2 b})$, if $\left(\overline{w^{\prime 2}}\right)^{\frac{1}{2}}$ is assumed to follow a Gaussian distribution with a width equal to $1.6 b_{w}$ (see Papanicolaou 1984) then

$$
\overline{\left(w^{\prime 2}\right)^{\frac{1}{2}}}=\left(\overline{w_{\mathrm{c}}^{\prime 2}}\right)^{\frac{1}{2}} \exp \left[-\left(\frac{r}{1.6 b_{w}}\right)^{2}\right] \text {. }
$$

This result, plus a Gaussian mean velocity profile, enables (8) to be integrated with $b(z) \rightarrow \infty$ to give

$$
m(z)=\frac{1}{2} \pi b_{w}^{2} \bar{w}_{\mathrm{c}}^{2}\left(1+\frac{(0.25)^{2}}{0.39}\right)=1.16 \frac{1}{2} \pi b_{w}^{2} w_{\mathrm{c}}^{2}
$$

This implies that the turbulence adds approximately $16 \%$ to the specific momentum flux of the mean flow. From the experimental results it is possible to determine the momentum flux of the mean advective flow normalized by the initial jet momentum flux $M$. This is plotted versus $z / D$ in figure 4 (PAP and VEL data). The data scatter is substantial but implies that $m(z) / M=0.96$. Therefore the total normalized momentum will be $m(z) / M=0.96+0.16=1.12$, which is $12 \%$ higher than the initial jet specific momentum flux. As shown in equation 9.111 of Fischer et al. (1979), the rate of change of flow force of a jet (which is primarily the momentum flux) is 


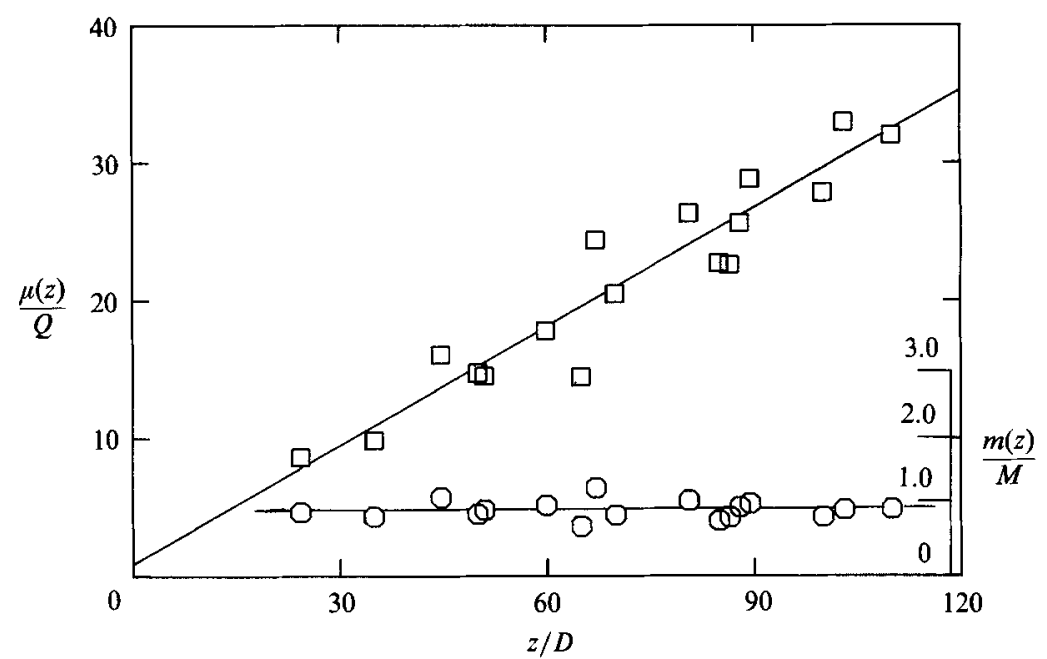

Frgure 4. Experimental values for the specific momentum flux $m(z)(\bigcirc)$ and volume flux $\mu(z)(\square)$ of a turbulent jet as a function of the distance $z / D$ from the origin.

controlled by the entrainment of ambient momentum. If the ambient fluid has no axial velocity then the momentum flux, i.e. flow force, is preserved. However, if there is any axial motion of the fluid external to the jet then the effect of the entrained momentum will not be negligible. For a jet in a closed tank, where there is a reverse flow near the tank walls and a weak coflow adjacent to the jet (Taylor 1958) then the momentum flux of the jet will increase along the jet axis because the first two terms on the right of equation 9.111 of Fischer et al. are positive (see also a similar argument by Kotsovinos 1978 for plane jets).

The mean-flow advective volume flux normalized with the initial jet volume flux, $\mu(z) / Q$, is also plotted versus $z / D$ in figure 4 . A linear fit to the data is

$$
\frac{\mu(z)}{Q}=0.284\left(\frac{z}{D}+3.3\right)
$$

Although Albertson et al. (1950) and Ricou \& Spalding (1961) reported a coefficient of 0.37 , Fischer et al. (1979) in a review of other experimental data suggest an average value of 0.282 , which is close to the value measured here.

\subsection{Mean flow in buoyant jets}

In the previous section the experimental measurements of the centreline-mean, r.m.s.-normalized velocity and concentration, and the jet width for a momentumdriven jet, were presented as functions of the dimensionless distance $z / D$ from the origin. The data are in reasonable agreement with the results of earlier investigations, implying that the experimental technique is adequate. In the following paragraphs the behaviour of a round buoyant jet for all three flow regimes-jets, plumes and transition - will be discussed. The results of a dimensional analysis for the two asymptotic flow regimes of jets and plumes will be compared with the experimental data.

The normalized time-averaged axial velocity and concentration on the axis of a buoyant jet versus the dimensionless elevation $z / I_{M}$ are plotted in figures $5(a)$ and 


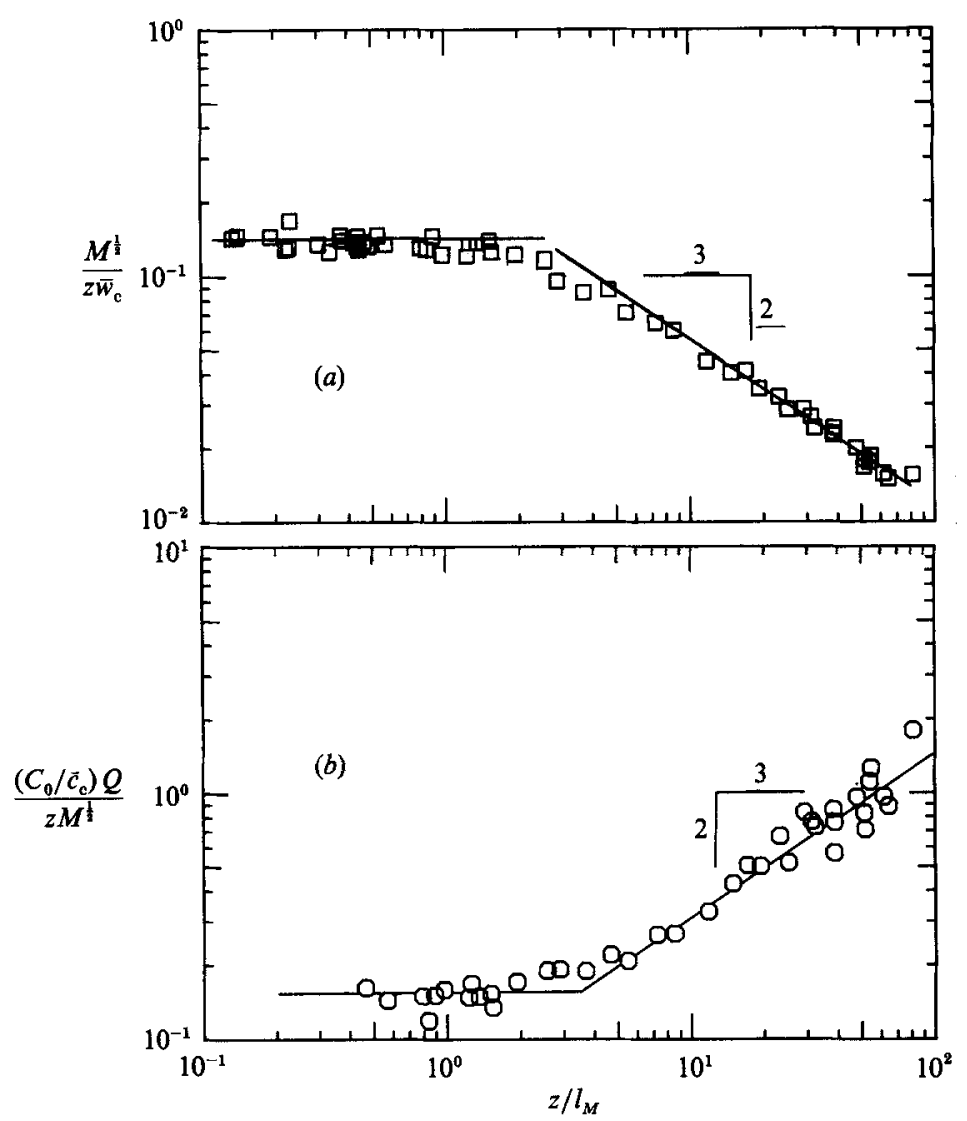

Figure 5. Non-dimensional $(a)$ mean velocity $(\square)$ and $(b)$ mean concentration $(O)$ decay along the axis of a buoyant jet plotted versus $z / l_{M}$.

5 (b) respectively. Following the dimensional analysis (Fischer et al. 1979) figure $5(a, b)$ implies

$$
\begin{gathered}
\frac{M^{\frac{1}{2}}}{z \bar{w}_{\mathrm{c}}}=0.132, \frac{S Q}{z M^{\frac{1}{2}}}=0.147 \text { for } \frac{z}{l_{M}}<1, \\
\frac{M^{\frac{1}{2}}}{z \bar{w}_{\mathrm{c}}}=0.260\left(\frac{z}{l_{M}}\right)^{-\frac{2}{3}}, \frac{S Q}{z M^{\frac{1}{2}}}=0.070\left(\frac{z}{l_{M}}\right)^{\frac{2}{3}} \text { for } \frac{z}{l_{M}}>5,
\end{gathered}
$$

where $S=C_{0} / \bar{e}_{\mathrm{c}}$. It is clear that the flow behaves like a jet for $z / l_{M}<1$ and like a plume for $z / l_{M}>5$. For $1<z / l_{M}<5$ there is a smooth transition from jets to plumes as the slope of the lines fitted to the logarithmic data changes from zero to $\pm \frac{2}{3}$. The results seem to indicate that a jet driven initially by both buoyancy and momentum will behave as a momentum jet up to a distance $z \approx l_{M}$ and as a plume beyond $5 l_{M}$ from its origin. This can help resolve the differences in the experimental constants defined by other studies of jets and plumes. Measurements close to the transition can be approximated by straight lines with constant slopes because of the data scatter and the transitional behaviour of the flow there. Thus a range of different experimental constants defining the decay of the mean velocity and concentration along the axis of the plume can be predicted. In the present experiments the decay constants are derived from a wide range of points extending well into both asymptotic regimes. 

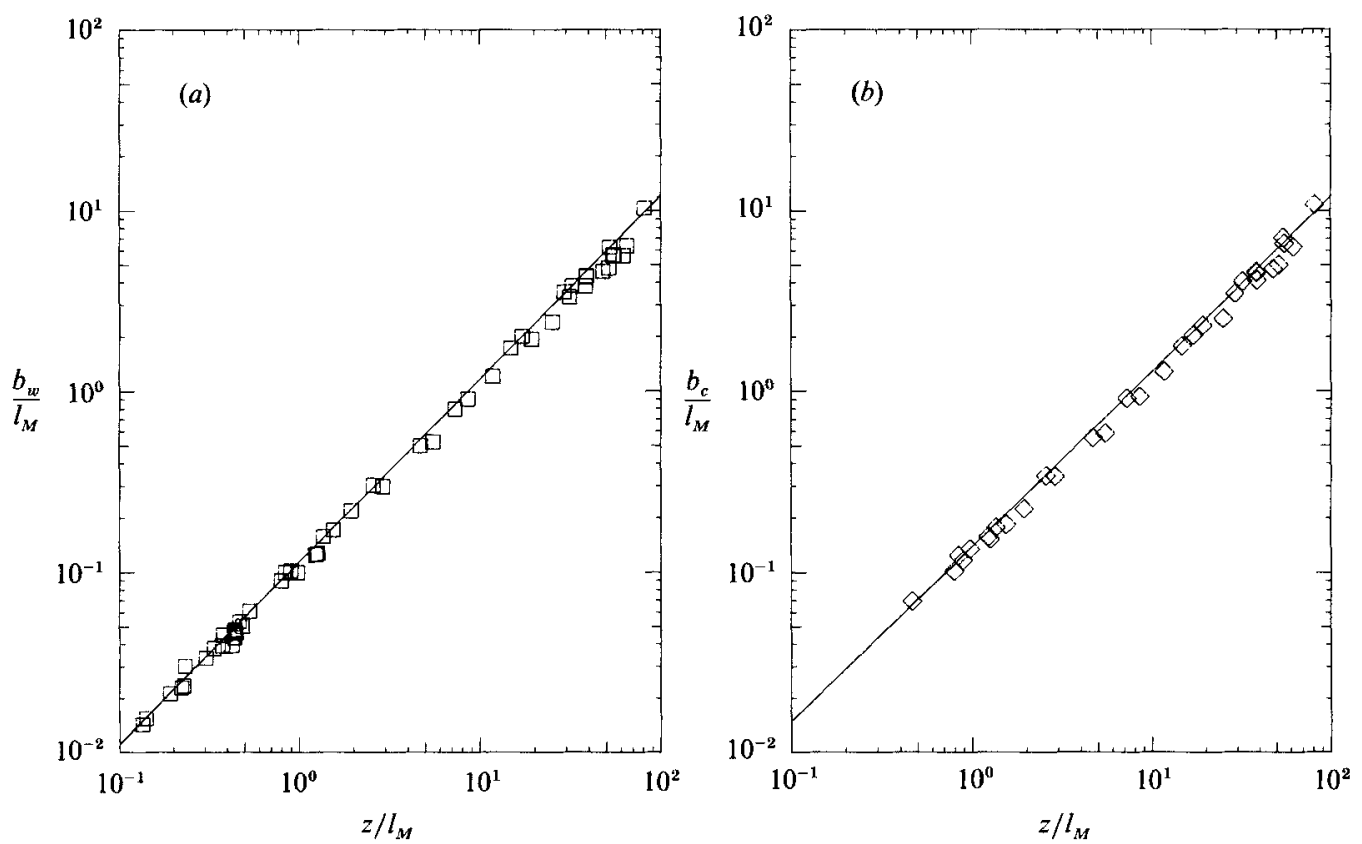

Figure 6. Non-dimensional $(a)$ velocity $1 /$ e-width $(\square)$ and $(b)$ concentration $1 /$ e-width $(\diamond)$ of a turbulent buoyant jet plotted versus $z / l_{M}$.

The velocity and concentration profile widths of a buoyant jet $b_{w}$ and $b_{c}$ normalized by the momentum lengthscale $l_{M}$ are plotted in figures $6(a, b)$ respectively as a function of $z / l_{M}$. The least-square fit to the velocity and concentration $1 / \mathrm{e}$ widths versus $z / l_{M}$ is

$$
\frac{b_{w}}{l_{M}}=0.108\left(\frac{z}{l_{M}}\right)^{0.995}, \frac{b_{c}}{l_{M}}=0.129\left(\frac{z}{l_{M}}\right)^{0.994},
$$

seeming to indicate a nonlinear growth for a buoyant jet. However, the exponents are very close to unity as predicted by previous dimensional arguments. Fischer et al. (1979), Papanicolaou \& List (1987) and others have reported that a plume is 'narrower' than a jet, a result indicative of nonlinear buoyant-jet growth. In the plume regime $\left(z / l_{M}>5\right)$ alone a least-square fit to the data points leads to the relations

$$
\frac{b_{w}}{l_{M}}=0.105 \frac{z}{l_{M}}, \quad \frac{b_{c}}{l_{M}}=0.112 \frac{z}{l_{M}} .
$$

The equations show clearly that $b_{c}$ is consistently larger than $b_{w}$ for both jets and plumes. Jet investigators have all reported that $b_{c}>b_{w}$ but plume experiments are equivocal. George et al. (1977) and Nakagome \& Hirata (1976) reported that $b_{w}>b_{c}$, while Rouse, Yih \& Humphreys, (1952) reported that $b_{c}>b_{w}$. However, these authors took measurements in the near plume field (less than 20 source diameters downstream) whereas the present investigation extended to 120 diameters in jets and 80 diameters in plumes. The results of the present work should therefore help resolve this question. Equations (15) imply that on average

$$
\lambda=\frac{b_{c}}{b_{w}}=1.194
$$



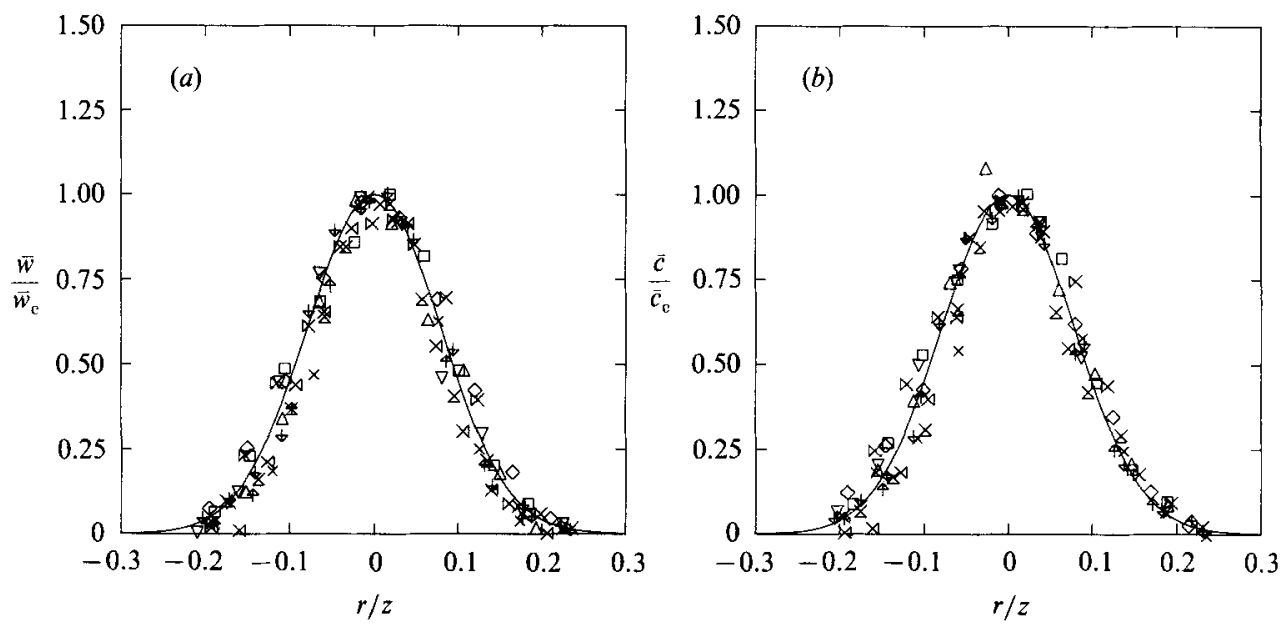

Figure 7. Non-dimensional $(a)$ mean velocity and $(b)$ mean concentration profile across a turbulent plume $z / l_{M}: X, 8.58 ; \uparrow, 11.77 ; \square, 14.80 ; \downarrow, 17.02 ; \diamond, 29.30 ; \mathbf{X}, 32.38 ; \triangle, 38.26 ; \mathbf{X}, 47.98 ; \nabla, 54.71 ;$ $\mathrm{X}, 61.87$.

over the range of $z / l_{M}$ of the present investigation, a value close to the 1.20 commonly used in the integral models used for design calculations of buoyant jets (Muellenhof et al. 1985).

The mean-velocity and concentration profiles normalized with the time-averaged centreline values are plotted versus $r / z$ in figure $7(a, b)$ for all experimental data with $z / l_{M}>5$ (plumes). There is some scatter to the data because of the wide range of $z / l_{M}$ of the data plotted. The dimensionless elevation $z / l_{M}$ varies from 8 (probably not a fully developed plume) to about 65 where the plume is presumably fully developed. Least-square Gaussian fits to the data plotted are

$$
\bar{w}=\bar{w}_{\mathrm{c}} \exp \left[-90(r / z)^{2}\right], \quad \bar{c}=\bar{c}_{\mathrm{c}} \exp \left[-80(r / z)^{2}\right] .
$$

The concentration profile agrees with those measured by Zimin \& Frik (1977) and Papanicolaou \& List (1987). However, Nakagome \& Hirata (1976), George et al. (1977) and Rouse et al. (1952) have reported wider concentration profiles than that of the present investigation.

An issue of importance to volcano and other geophysical modellers are the rates of entrainment of ambient fluid, usually defined as an entrainment coefficient. Using the continuity equation (Fischer et al. 1979), and substituting the mean-centrelinevelocity decay laws from the present experimental work $W / \bar{w}_{\mathbf{c}}=0.149 z / D$ and $b_{w}=0.109 z$ in jets gives the jet entrainment coefficient $a_{\mathrm{j}}=0.0545$. For a plume, substituting $\bar{w}_{\mathrm{c}}=3.85 B^{\frac{1}{3}} z^{-\frac{1}{3}}$ from equation (13) and $b_{w}=0.105 z$ into the continuity equation, implies that $a_{\mathrm{p}}=0.0875$. The values proposed by Fischer et al. (1979) for these respective entrainment coefficients are 0.0533 and 0.0833 .

The local Richardson number in a buoyant jet, defined as

$$
R(z)=\frac{\mu \beta^{\frac{1}{2}}}{m^{\frac{5}{4}}}
$$

provides an estimate of the local degree of plume-like behaviour for the flow where parameters $\mu(z), \beta(z)$ and $m(z)$ are the estimates of the volume flux and specific buoyancy and specific momentum flux of the mean flow at a distance $z$ from the jet 


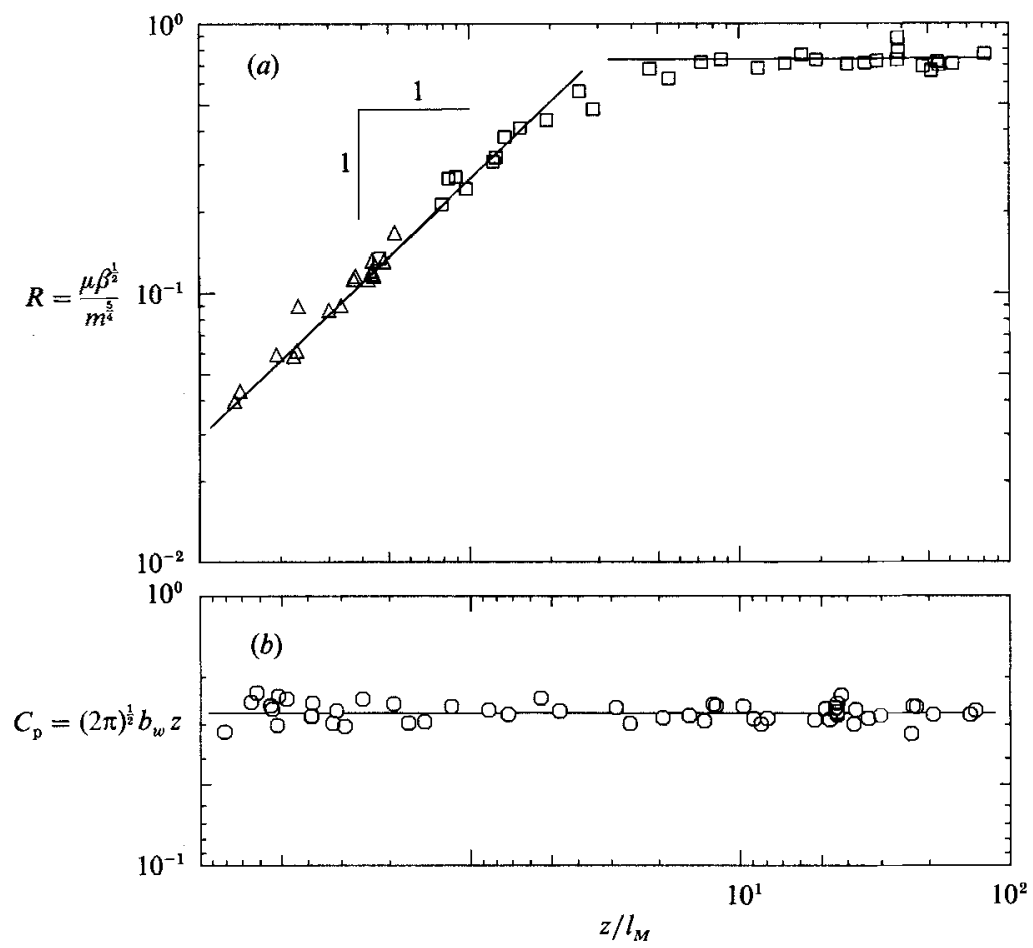

FigURE 8. (a) Richardson number in a turbulent buoyant jet and $(b)$ round plume width parameter plotted against $z / l_{M}$.

origin. Assuming Gaussian mean velocity and concentration profiles, $\lambda=b_{c} / b_{w}$, and that the density distribution is given by the tracer distribution, and substituting into integrals for the volume, momentum and buoyancy fluxes for the mean flow gives

$$
R(z)=\left[\frac{4(2 \pi)^{\frac{1}{2}} \lambda^{2}}{1+\lambda^{2}} g \frac{(\Delta \rho)_{0}}{\rho_{\mathrm{a}}} \frac{\bar{c}_{\mathrm{c}}}{C_{0}} \frac{b_{w}}{{\overline{w_{\mathrm{c}}}}^{2}}\right]^{\frac{1}{2}} .
$$

Similarly, a local jet-width parameter $C_{\mathrm{p}}$ is defined as

$$
C_{\mathrm{p}}(z)=\frac{\mu}{m^{\frac{1}{2}} z}=(2 \pi)^{\frac{1}{2}} b_{w} z^{-1}
$$

Both $R(z)$ and $C_{\mathrm{p}}(z)$ are plotted versus $z / l_{M}$ in figure $8(a)$ and $8(b)$ respectively. In figure $8(a)$ the squares correspond to Richardson numbers calculated from the simultaneous velocity and concentration measurement experiments (EXP). The triangles in the jet regime were calculated from the velocity measurement experiments only (VEL and PAP) using $\lambda=1.2$ and $C_{0} / \bar{c}_{\mathrm{c}}=0.157 z / D$ determined earlier. Dimensional arguments (List \& Imberger 1973 ) imply that $R(z) \approx z / l_{M}$ in jets and $R(z)=R_{\mathrm{p}}=$ constant in plumes, and the $C_{\mathrm{p}}$ must be constant in both jets and plumes. The data points in figure $8(a, b)$ appear to verify the asymptotic analysis although the mean values of $C_{\mathrm{p}}$ and $R_{\mathrm{p}}$ are 0.27 and 0.716 respectively, higher than 0.25 and 0.557 proposed by Fischer et al. (1979). Figure $8(a)$ appears to confirm that the plume-like behaviour occurs for $z / l_{M}>5$ since the local Richardson number takes a limiting value in this region.

The local volume flux and specific momentum flux $\mu(z)$ and $m(z)$ respectively, for 


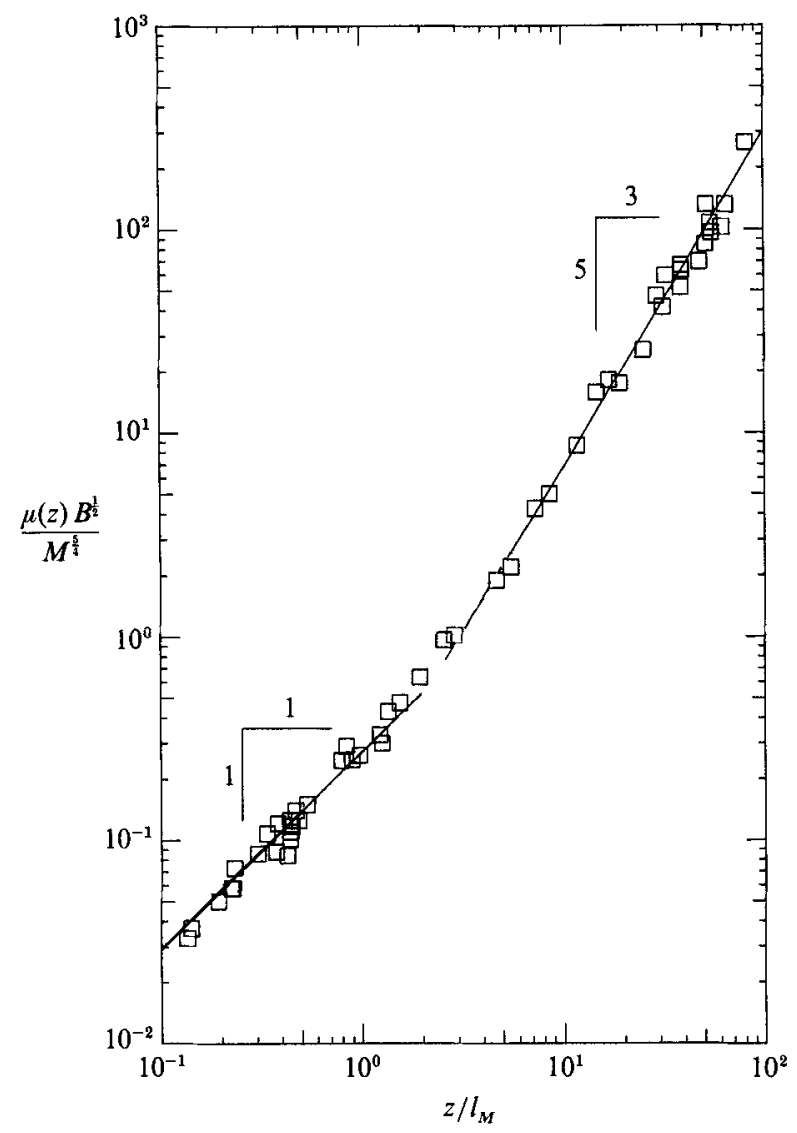

FIGURE 9. Volume flux in a turbulent buoyant jet plotted against the non-dimensional distance $z / l_{M}$ from the jet origin.

the mean flow at a distance $z$ from the jet origin, can be calculated from the mean velocity profiles. The development of these fluxes in the transition from jets to plumes is best presented in a dimensionless formulation that displays quite clearly where the transition occurs. The volume flux $\mu(z)$ is normalized as $\mu(z) B^{\frac{1}{2}} / M^{\frac{5}{4}}$, and when this is plotted against $z / l_{M}$ it is clear that jet behaviour is apparent for $z / l_{M}<1$ and plume growth occurs for $z / l_{M}>5$ as shown in figure 9 . The jet line is given by (11) and the plume line by

$$
\frac{\mu(z) B^{\frac{1}{2}}}{M^{\frac{5}{4}}}=0.140\left(\frac{z}{l_{M}}\right)^{\frac{5}{3}} .
$$

Similarly, the momentum flux also shows the transition explicitly, as depicted in figure 10. The jet line is $m(z) / M=0.96$ as before, and the plume line is

$$
\frac{m(z)}{M}=0.290\left(\frac{z}{l_{M}}\right)^{\frac{4}{3}}
$$

The constant of proportionality for plumes given above is less than the $\mathbf{0 . 3 5 0}$ suggested by Fischer et al. (1979), who calculated this constant from Rouse et al. (1952) data which lie close to the transition region. 


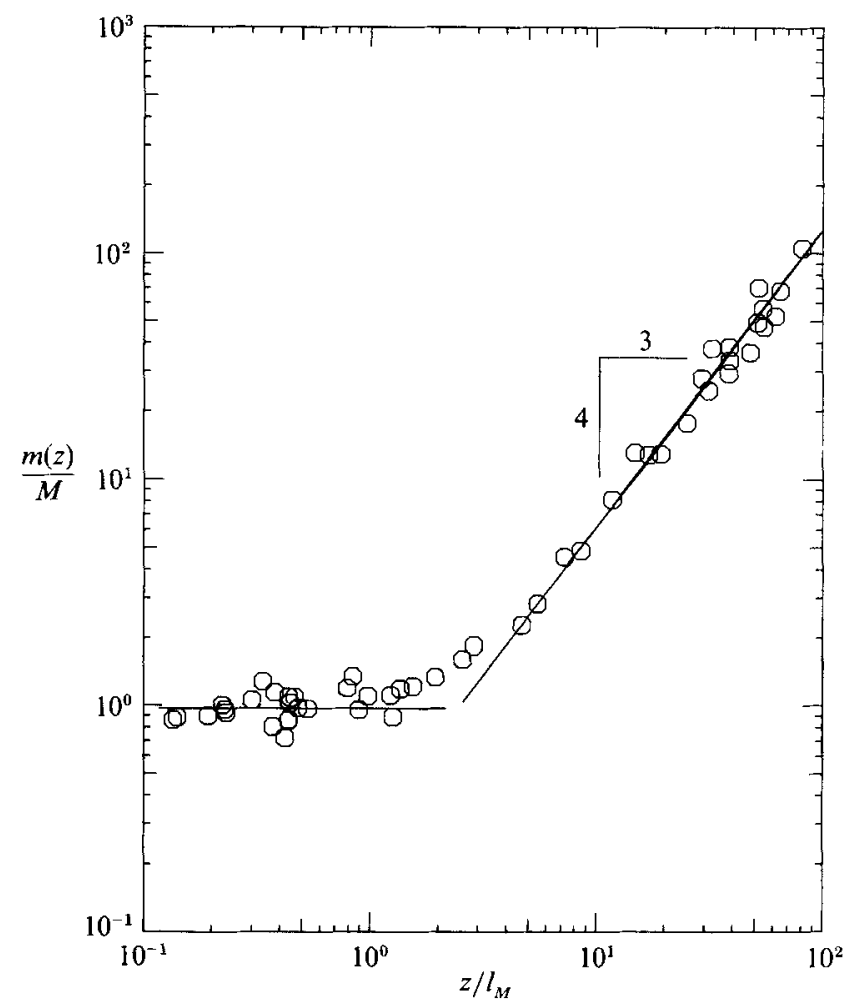

Figure 10. Momentum flux in a turbulent buoyant jet plotted against the non-dimensional distance $z / l_{M}$ from the jet origin.

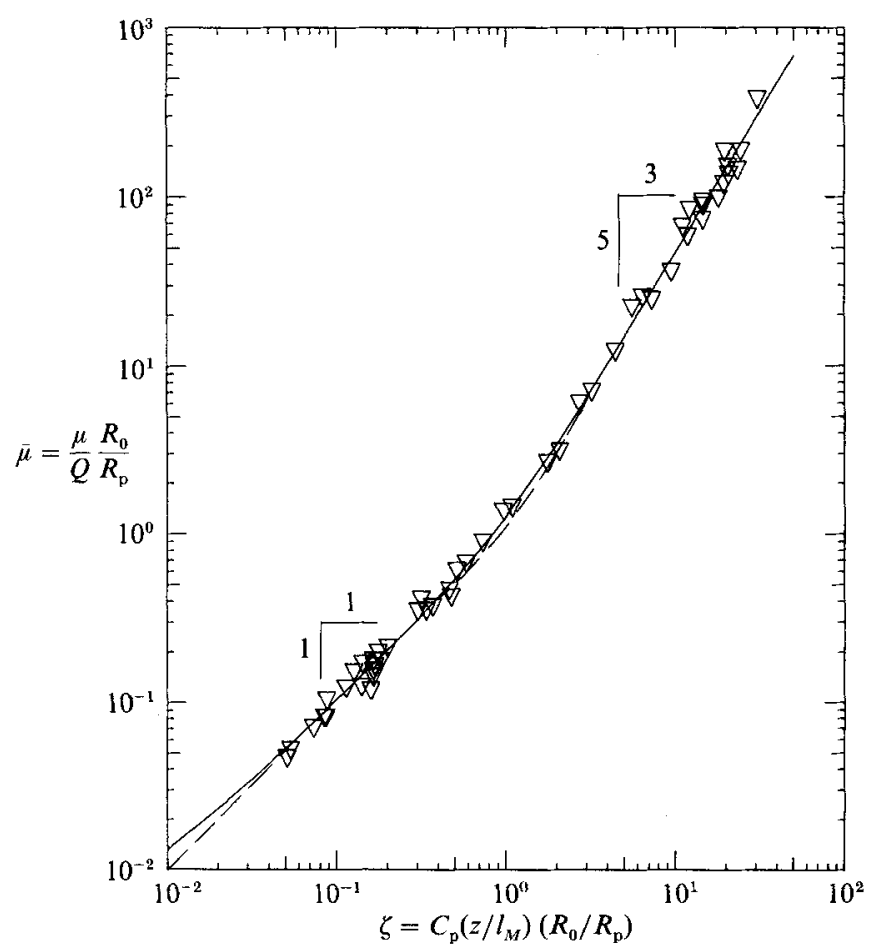

Fiaure 11. Mean dilution $\bar{\mu}$ in turbulent buoyant jets against the non-dimensional elevation $\zeta$. 
An analysis by Kotsovinos \& List (1976) suggested that the dimensionless dilution $\bar{\mu}=(\mu / Q)\left(R_{0} / R_{\mathrm{p}}\right)$ is a function of the dimensionless elevation $\zeta=\left(C_{\mathrm{p}} / R_{\mathrm{p}}\right)\left(z / l_{M}\right)$ of the form

$$
\bar{\mu}=\left(\zeta+\overline{R_{0}}\right)\left[1-\overline{R_{0}}+\left(\zeta+\overline{R_{0}^{\frac{1}{2}}}\right)^{2}\right]^{\frac{1}{3}},
$$

with initial Richardson number $\overline{R_{0}}=R_{0} / R_{\mathrm{p}} \approx 10^{-5}$. The experimental results obtained here and plotted in figure 11 are in remarkable agreement with this prediction from analysis.

\section{Turbulence properties}

\subsection{Preliminary analysis}

Dimensional analysis will be used to provide a basis for presentation of the turbulence properties measured in the experiments. If the assumption is made that the radial distribution of the cross-correlation term $\overline{w^{\prime} c^{\prime}}$ is self-similar in jets and plumes then it can be written as

$$
\overline{w^{\prime} c^{\prime}}=\left(\overline{w^{\prime} c^{\prime}}\right)_{\mathrm{c}} f\left(\frac{r}{z}\right)
$$

where the primes denote the deviation from the mean values and $w$ is the axial velocity and $c$ the concentration. $r$ and $z$ are the radial and axial coordinates, the subscript $c$ denotes values along the axis and $f$ is some shape function. In a jet, when the 'motionless' ambient fluid is entrained and mixed with the jet fluid, it reduces the jet mean velocity (momentum conservation) and dilutes the concentration of a tracer transported by the jet fluid (mass conservation). Hence it seems a reasonable assumption that $w$ and $c$ are correlated in a jet so that

$$
\overline{w^{\prime} c^{\prime}} \sim \bar{w}\left(\overline{c^{\prime 2}}\right)^{\frac{1}{2}}, \quad \overline{w^{\prime} c} \sim \bar{c}\left(\overline{w^{\prime 2}}\right)^{\frac{1}{2}},
$$

meaning that the decay of the transport term $\overline{w^{\prime} c^{\prime}}$ follows the decay of the turbulence intensity of either the velocity, or the concentration, in a self-similar jet. Similarly, in a plume, if the ambient fluid is entrained and mixed with the jet fluid, a local source of buoyancy (density fluctuation) at a point in the flow should result in a local velocity fluctuation. Assuming that a local density difference is proportional to the concentration of tracer at that point we can replace $c^{\prime}$ by $\rho^{\prime}$ in plume flow to write

$$
\text { or alternatively, } \quad \overline{w^{\prime} c^{\prime}} \sim\left\{\begin{array}{c}
\bar{c}\left(\overline{w^{\prime 2}}\right)^{\frac{1}{2}} \\
\Delta \bar{\rho}\left(\overline{w^{\prime 2}}\right)^{\frac{1}{2}},
\end{array}\right.
$$

In a jet, the radial distributions of $\bar{w}$ and $\bar{c}$ at any distance $z$ from the flow source are known to be proportional to $\bar{w}_{\mathrm{c}}$ and $\bar{c}_{\mathrm{c}}$ respectively. Hence in the above equations (25) and (26) the mean values $\bar{w}$ and $\bar{c}$ could be replaced by $\bar{w}_{\mathrm{c}}$ and $\bar{c}_{\mathrm{c}}$, and from the preceding results for mean flows we obtain

$$
\overline{w^{\prime} c^{\prime}} \sim\left\{\begin{array}{l}
\bar{w}_{\mathrm{c}}\left(\overline{c^{\prime 2}}\right)^{\frac{1}{2}} \sim \frac{M^{\frac{1}{2}}}{z}\left(\overline{c^{\prime 2}}\right)^{\frac{1}{2}} \\
\bar{c}_{\mathrm{c}}\left(\overline{w^{\prime 2}}\right)^{\frac{1}{2}} \sim \frac{C_{0} Q}{z M^{\frac{1}{2}}}\left(\overline{w^{\prime 2}}\right)^{\frac{1}{2}}
\end{array}\right.
$$


for jets and

$$
\overline{w^{\prime} c^{\prime}} \sim\left\{\begin{array}{c}
\frac{M^{\frac{1}{2}}}{z}\left(\frac{z}{l_{M}}\right)^{\frac{2}{3}}\left(\overline{c^{\prime 2}}\right)^{\frac{1}{2}} \\
\overline{\left(\bar{w}^{\prime 2}\right)^{\frac{1}{2}}} \frac{C_{0} Q}{z M^{\frac{1}{2}}}\left(\frac{z}{l_{M}}\right)^{-\frac{2}{3}},
\end{array}\right.
$$

for plumes. The tracer conservation equation for a jet can be written

$$
Q C_{0}=\int_{\text {jet }} \overline{w c} 2 \pi r \mathrm{~d} r=\int_{\text {jet }} \overline{w c} 2 \pi r \mathrm{~d} r+\int_{\text {jet }} \overline{w^{\prime} c^{\prime}} 2 \pi r \mathrm{~d} r .
$$

For the limiting cases of a jet and a plume, the width $b(z) \sim z$ and the mean velocity and concentration profiles are self-similar and approximated by Gaussian distributions, hence

$$
\int_{\text {jet }} \overline{w c} 2 \pi r \mathrm{~d} r \sim \bar{w}_{\mathrm{c}} \bar{c}_{\mathrm{c}} \pi z^{2}
$$

From (27) and (28) then

$$
\int_{\text {jet }} \overline{w^{\prime} c^{\prime}} 2 \pi r \mathrm{~d} r \sim\left\{\begin{array}{l}
\bar{w}_{\mathrm{c}} \pi z^{2}\left(\overline{c_{\mathrm{c}}^{2}}\right)^{\frac{1}{2}} \\
\bar{c}_{\mathrm{c}} \pi z^{2}\left(\overline{w_{\mathrm{c}}^{\prime 2}}\right)^{\frac{1}{2}}
\end{array}\right. \text { or }
$$

Following (30) and (31), (29) can be written as

$$
Q C_{0}=J_{1} \bar{w}_{\mathrm{c}} \bar{c}_{\mathrm{c}} \pi z^{2}+J_{2} \bar{w}_{\mathrm{c}}\left(\overline{c_{\mathrm{c}}^{\prime 2}}\right)^{\frac{1}{2}} \pi z^{2},
$$

where $J_{1}$ and $J_{2}$ are constants representing profile integrals. Equations (27) and (32) then lead to

or

$$
\begin{aligned}
Q C_{0} & =J_{1} \frac{M^{\frac{1}{2}}}{z} \frac{C_{0} Q}{z M^{\frac{1}{2}}} \pi z^{2}+J_{2} \frac{M^{\frac{1}{2}}}{z}\left(\overline{c_{\mathrm{c}}^{\prime 2}}\right)^{\frac{1}{2}} \pi z^{2} \\
1 & =\pi J_{1}+\pi J_{2} M^{\frac{1}{2}}\left(\overline{c_{\mathrm{c}}^{\prime 2}}\right)^{\frac{1}{2}} z \\
& =\pi J_{1}+\pi J_{2}\left(\overline{u_{\mathrm{c}}^{\prime 2}}\right)^{\frac{1}{2}} \frac{C_{0} Q}{z(M)^{\frac{1}{2}}} z^{2},
\end{aligned}
$$

which indicates that $\left(\overline{c_{\mathrm{c}}^{\prime 2}}\right)^{\frac{1}{2}} \sim 1 / z$ and $\left(\overline{w_{\mathrm{c}}^{\prime 2}}\right)^{\frac{1}{2}} \sim 1 / z$. Therefore the r.m.s. velocity and concentration along the jet axis follow the same decay laws as their mean values. Similarly in a plume,

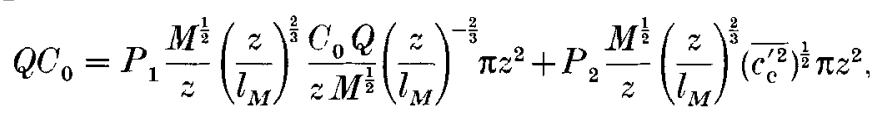

leading to $\left(\overline{c_{\mathrm{c}}^{\prime 2}}\right)^{\frac{1}{2}} \sim z^{-\frac{5}{8}}$, or

$$
Q C_{0}=P_{1} \frac{M^{\frac{1}{2}}}{z}\left(\frac{z}{l_{M}}\right)^{\frac{2}{3}} \frac{C_{0} Q}{z M^{\frac{1}{2}}}\left(\frac{z}{l_{M}}\right)^{-\frac{2}{3}} \pi z^{2}+P_{2}\left(\overline{w_{\mathrm{c}}^{\prime 2}}\right)^{\frac{1}{2}} \frac{C_{0} Q}{z M^{\frac{1}{2}}}\left(\frac{z}{l_{M}}\right)^{-\frac{2}{3}} \pi z^{2},
$$

implying that $\left(\overline{w_{\mathrm{c}}^{\prime 2}}\right)^{\frac{1}{2}} \sim z^{-\frac{1}{3}}$, where $P_{1}$ and $P_{2}$ are (unknown) profile integrals. Hence the r.m.s. axial velocity and concentration appear to follow the same decay laws as their mean values along the plume axis. The r.m.s. velocity and concentration normalized by their corresponding mean values necessarily then have to be constant along the axis of motion, where self-similarity is assumed both in jets and plumes. 


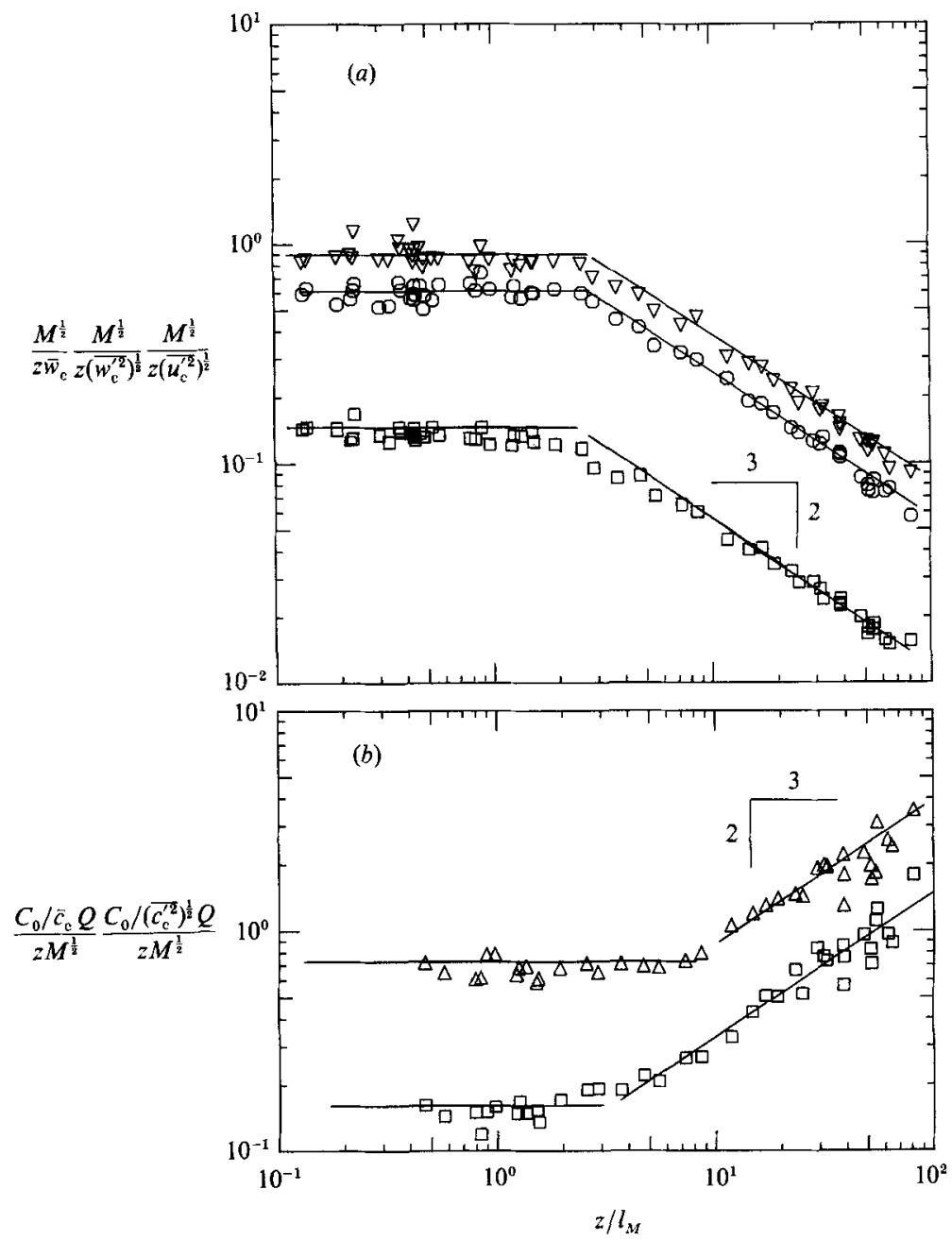

FIgurf 12. (a) Non-dimensional velocity distribution along the axis of a buoyant jet versus the dimensionless elevation $z / l_{M}$ from the origin: $\square$, mean velocity; $\bigcirc$, axial turbulent intensity; $\nabla$, radial turbulent intensity. (b) Non-dimensional concentration distribution along the axis of a buoyant jet; $\square$ mean concentration, $\triangle$ turbulence intensity.

\subsection{Experimental results}

The above analysis is confirmed by the experimental data, which show that the normalized r.m.s. axial and radial velocities and concentration on the jet axis take a constant value beyond 50 jet diameters from the flow source. More specifically the normalized turbulent intensities in the self-similar regime of a jet are

$$
\frac{\left(\overline{w_{\mathrm{c}}^{\prime 2}}\right)^{\frac{1}{2}}}{\bar{w}_{\mathrm{c}}}=0.25, \quad \frac{\left(\overline{u_{\mathrm{c}}^{\prime 2}}\right)^{\frac{1}{2}}}{\bar{w}_{\mathrm{c}}}=0.17, \quad \frac{\left(\overline{c_{\mathrm{c}}^{\prime}}\right)^{\frac{1}{2}}}{\bar{c}_{\mathrm{c}}}=0.22
$$

as shown in figure 2. The corresponding results for plumes are plotted against the dimensionless distance from the origin, $z / l_{M}$, in figure $12(a, b)$. The data confirm that the r.m.s. velocities and concentration have the same decay characteristics as the 
corresponding time-averaged mean values, as defined by the dimensional analysis. Specifically,

$$
\begin{aligned}
& \frac{M^{\frac{1}{2}}}{z\left(\overline{w_{\mathrm{c}}^{\prime 2}}\right)^{\frac{1}{2}}}= \begin{cases}0.57 & \text { for } \frac{z}{l_{M}}<1 \\
1.13\left(\frac{z}{l_{M}}\right)^{-\frac{2}{3}} & \text { for } \frac{z}{l_{M}}>5\end{cases} \\
& \frac{M^{\frac{1}{2}}}{z\left(\overline{u_{\mathrm{c}}^{\prime 2}}\right)^{\frac{1}{2}}}= \begin{cases}0.87 & \text { for } \frac{z}{l_{M}}<1 \\
1.73\left(\frac{z}{l_{M}}\right)^{-\frac{2}{3}} & \text { for } \frac{z}{l_{M}}>5,\end{cases} \\
& \frac{C_{0} /\left(\overline{c_{\mathrm{c}}^{\prime 2}}\right)^{\frac{1}{2}} Q}{z M^{\frac{1}{2}}}= \begin{cases}0.68 & \text { for } \frac{z}{l_{M}}<1 \\
0.18\left(\frac{z}{l_{M}}\right)^{\frac{2}{3}} & \text { for } \frac{z}{l_{M}}>5 .\end{cases}
\end{aligned}
$$

Figure 12 shows that there is an abrupt transition occurring around $z / l_{M}=2.5$ for the r.m.s. velocities and at about $z / l_{M}=8.0$ for the r.m.s. concentration. This is not so apparent in mean velocity and concentration data.

The normalized r.m.s. axial and radial velocity profiles in jets and plumes are plotted in figures $13(a)$ and $13(b)$ respectively. Given the different mechanics responsible for the turbulence it is remarkable both how similar and self-similar the results are in each case. Wygnanski \& Fiedler (1969) reported relative radial and tangential turbulent-jet velocity intensities that were equal but slightly smaller than the axial intensity. The present investigation shows a much smaller turbulence intensity for the radial velocity. In these experiments the jet turbulence may not have been as fully developed as that of Wygnanski \& Fiedler (1969) since the initial jet Reynolds numbers (3000-11000) were much lower than Wygnanski \& Fiedler's $\left(10^{5}\right)$. Another reason may be the size of the Doppler scattering volume used in the measurement of the radial velocity component. It was three times as long as that for the axial velocity measurement and therefore would cause a loss in the resolution, and signal smoothing, with a lower apparent turbulent intensity. The results can also be compared with the following: Abbiss, Bradbury \& Wright (1975) 0.28 and 0.22 (normalized axial and radial r.m.s. velocities); Antonia, Chambers \& Hussain (1980) 0.228 and 0.25 for the centreline and peak axial r.m.s. velocity; Birch et al. (1978) 0.18 and 0.28 (centreline and peak); Chevray \& Tutu (1978) 0.23 and 0.19 (axial and radial); Corrsin \& Uberoi (1949) 0.22 and 0.175. In addition, Corrsin (1943), Hinze \& van der Hegge Zijnen (1949) and Sforza \& Mons (1978) all reported axial r.m.s. velocities of 0.25 . Rosler \& Bankoff (1963) measured an air and a water jet at the same Reynolds number and reported turbulence intensities of 0.22 and 0.32 respectively. Gibson (1963) and Antonia et al. (1975) found the same axial and radial turbulent intensity of 0.30 at the jet axis. Plume investigators Nakagome \& Hirata (1976) and George et al. (1977) found centreline axial turbulent intensities from 0.20 to 0.30 with an average value of 0.25 , which agrees with the present investigation, although their measurements were limited to only 11 and 16 jet diameters downstream respectively. Kotsovinos (1977) reported an axial turbulent velocity in a two-dimensional plume of about 0.50 , or almost twice the jet value. The present authors believe that the explanation for this exceptionally high value is 'pseudoturbulence' due to temperature-induced refractive index changes in Kotsovinos' 

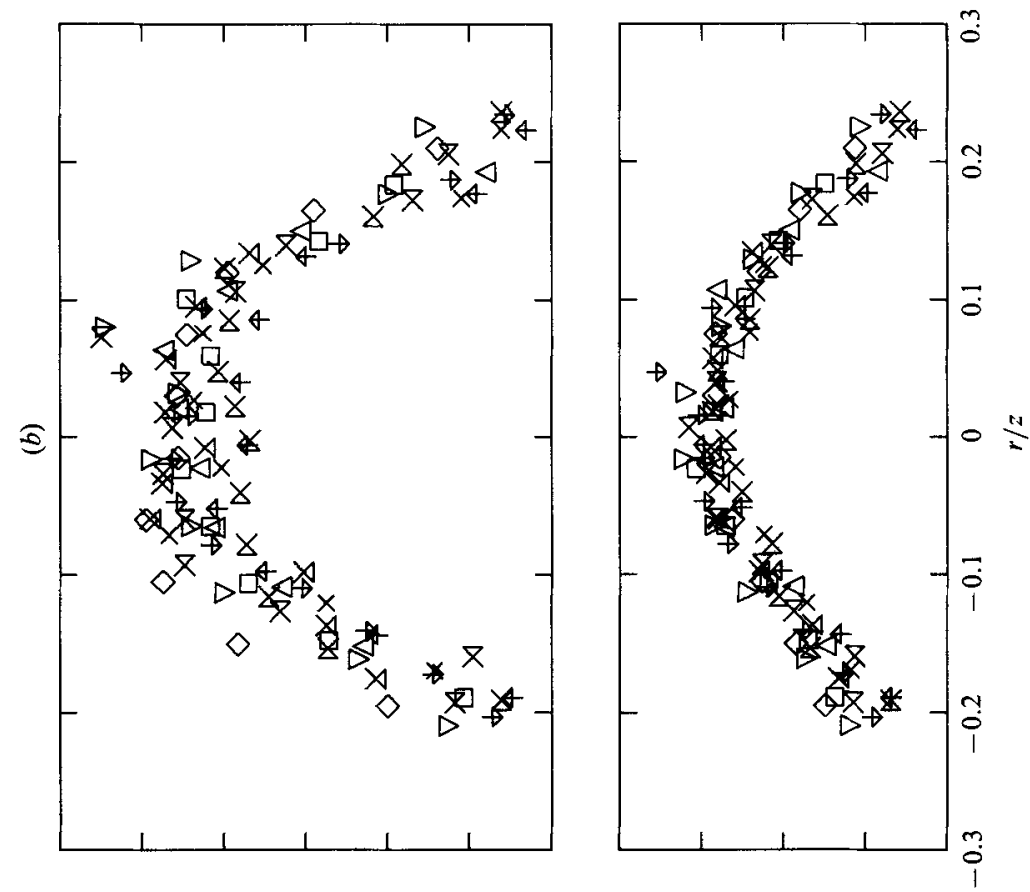

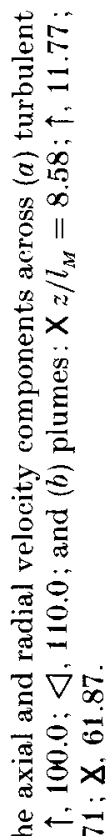

$\underset{0}{0} \frac{1}{\infty}$
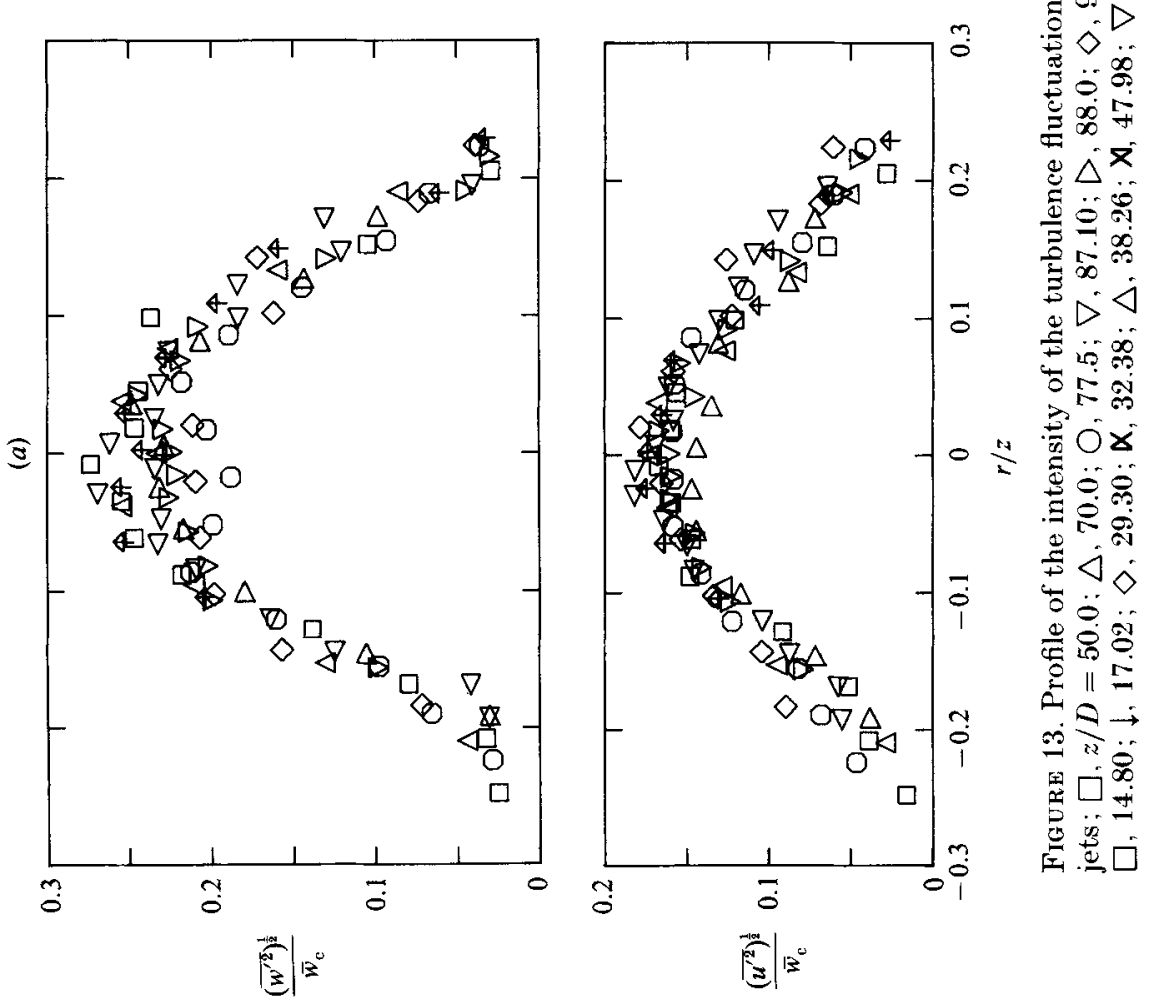


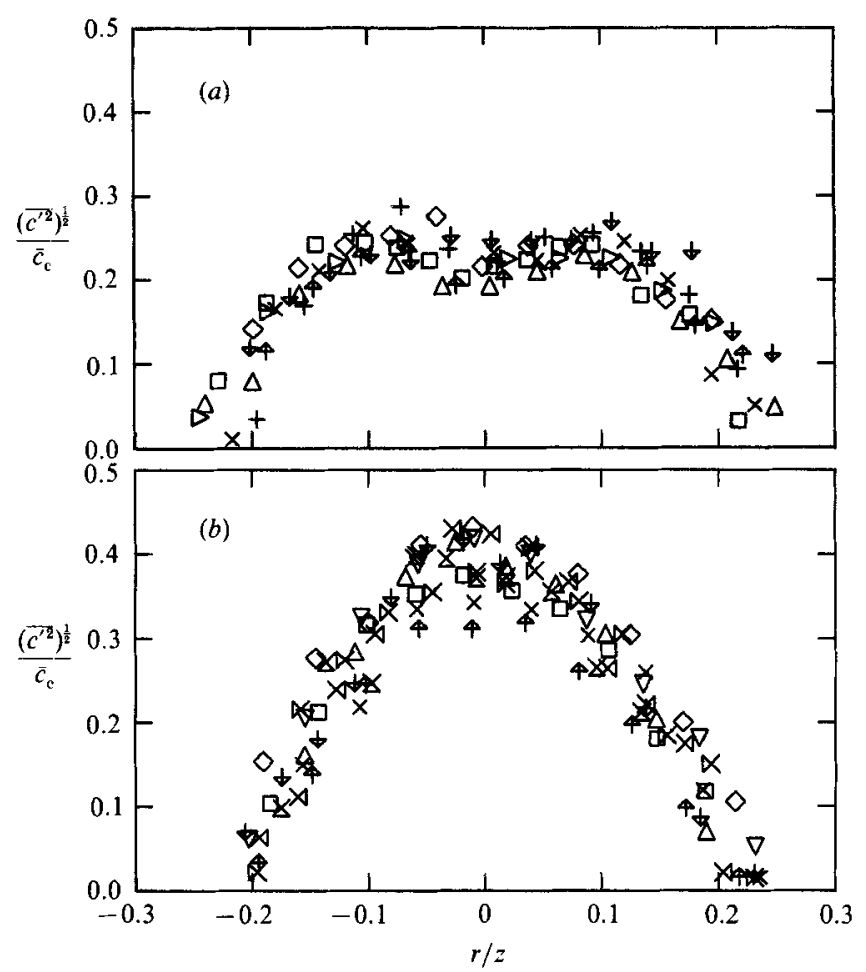

Figure 14. Profile of the intensity of turbulence of the concentration (a) across a turbulent jet: $\triangle$, $z / D=32.80 ; D, 45.47 ; \square, 47.87 ;+, 64.80 ; X, 71.47 ; \downarrow, 77.47 ; \uparrow, 84.93 ; \diamond, 98.13 ;(b)$ across a plume (symbols as in figure $13 b$ ).

plume. His experimental set-up which used temperature as a tracer, caused the LDV reference beam to wobble, resulting in an interrupted photomultiplier-tube velocity signal, which was interpreted as turbulence. Kotsovinos did not evaluate the noise level in his turbulence velocity signal from this source. Tests such as performed by Mizushina et al. (1979) and those described in the Appendix would possibly have resolved this question. It is believed that the results presented here are free from this bias. Ramaprian \& Chandrasekhara (1983), in their studies of two-dimensional plumes, did report a higher turbulence intensity in plumes $(0.25-0.30)$ than in jets, 0.20 , although their investigations must not have been totally free of noise problems in the plume regime. They found a noise level at the boundary (zero-level turbulence) from 0.03 in jets to 0.08 in plumes.

Although the r.m.s. velocity profiles in plumes and jets appear similar, figure $14(a, b)$ indicate that the normalized r.m.s. concentration for Rhodamine $6 \mathrm{G}$ dye in jets is quite different from that in plumes. In jets the measured centreline normalized r.m.s. concentration is 0.22 with a peak value of 0.25 occurring at a radial distance $r / z=0.10$. The result agrees with that reported by Becker et al. (1967), Antonia et al. (1980), Birch et al. (1978), Chevray \& Tutu (1978) and Grandmaison et al. (1977). However, Corrsin \& Uberoi (1949), Rosenweig, Hottel \& Williams (1961), Wilson \& Dankwerts (1964), and Papanicolaou \& List (1987) have reported lower r.m.s. centreline concentrations or temperatures. Most of the measurements by the above authors, except for those by Wilson \& Dankwerts and Grandmaison et al. were made within $\mathbf{4 5}$ jet diameters from the nozzle where jet flow is still not fully developed. 
Further downstream, at $z / D=59$, Antonia et al. (1975) reported values of 0.255 and 0.330 (centreline and peak). In a plume, the concentration turbulent intensity was higher than in a jet, with the peak at the plume axis. The centreline value is about 0.40, substantially higher than the 0.20-0.25 measured in jets. This agrees with results reported by Papanicolaou \& List (1987) and Kotsovinos (1985). George et al. (1977) and Nakagome \& Hirata (1976) report values of 0.250 .40 from measurements performed in non-fully developed flow.

\subsection{Turbulent transport}

The cross-correlation of the turbulent fluctuations of the velocities and the concentration can provide information important to the modelling of turbulent shear flows. Measurement of this correlation is difficult with intrusive instrumentation, especially hot wires and films that do not resolve the direction of the flow. This is particularly important in the boundary regions of the flow where frequent flow reversals are measured with laser-Doppler systems. In this experiment the discrete calibrated signals for $w, u$ and $c$ were used to calculate the cross-correlations as follows:

$$
\left.\begin{array}{l}
\overline{w^{\prime} u^{\prime}}=\frac{1}{N} \sum_{i=1}^{N} w_{i}(r, z) u_{i}(r, z)-\bar{w}(r, z) \bar{u}(r, z), \\
\overline{w^{\prime} c^{\prime}}=\frac{1}{N} \sum_{i=1}^{N} w_{i}(r, z) c_{i}(r, z)-\bar{w}(r, z) \bar{c}(r, z), \\
\overline{u^{\prime} c^{\prime}}=\frac{1}{N} \sum_{i=1}^{N} u_{i}(r, z) c_{i}(r, z)-\bar{u}(r, z) \bar{c}(r, z),
\end{array}\right\}
$$

with $i=1,2, \ldots, N$, where $N$ is the number of data points sampled at a point $(r, z)$. The $w, u$ correlation (turbulent shear stress) normalized by the mean centreline velocity squared, $\overline{w^{\prime} u^{\prime}} / \bar{w}_{\mathbf{c}}^{2}$, (not plotted) is similar in both jets and plumes and the maximum dimensionless shear stress $(0.015)$ occurred at about $r / z=0.09$. This is lower than the 0.02 reported for jets by Abbiss et al. (1975), 0.035 by Antonia et al. (1975) and 0.016-0.017 reported by Rosler \& Bankoff (1963) and Wygnanski \& Fiedler (1969) (although Corrsin \& Uberoi (1949) reported a value of 0.011 ). The low value in this study is attributed to the higher measured mean centreline velocity as discussed previously. No plume shear-stress measurements have been reported by others.

The axial normalized turbulent tracer flux $\overline{w^{\prime} c^{\prime}} / \bar{w}_{\mathrm{c}} \bar{c}_{\mathrm{c}}$ profile is plotted in figure $15(a, b)$ for jets and plumes respectively. Although there is significant scatter the distributions appear to be quite different in each case and similar to the profiles of the r.m.s. concentration distributions in figure $14(a, b)$. In jets the maximum value appears to be about 0.020 whereas in plumes the maximum value is about 0.050 . This is congruent with the values previously reported for jets by Antonia et al. (1975) (0.030), Grandmaison et al. (1977) (0.026) and Chevray \& Tutu (1978) (0.021). In axisymmetric plumes, Nakagome \& Hirata (1976) and George et al. (1977) reported 0.040 and 0.060 respectively. The normalized radial turbulent tracer transport $\overline{u^{\prime} c^{\prime}} / \bar{w}_{\mathrm{c}} \bar{c}_{\mathrm{c}}$ when plotted against $r / z$ (not presented here) shows a peak value at around $r / z=0.10$ of 0.015 for jets and about $0.020-0.025$ in plumes. Antonia et al. (1975) give a much higher peak value of 0.041 and Corrsin \& Uberoi (1949) and Chevray \& Tutu (1978) report peak values of 0.0125 and 0.016 respectively in jets. There are no other measurements in plumes.

In practical models of jets and plumes used in the engineering profession the 


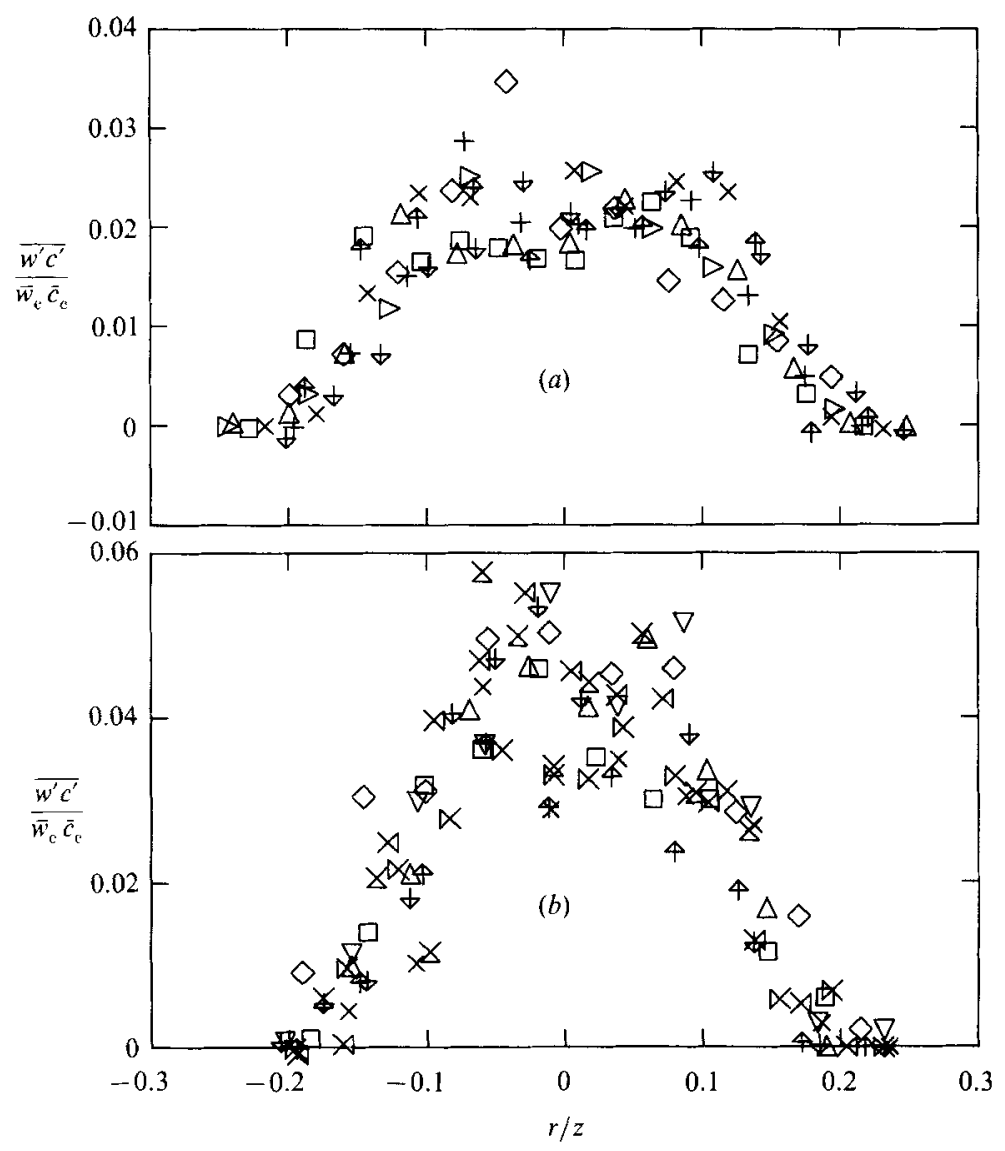

Figure 15. Normalized axial turbulent tracer flux profile $(a)$ across a turbulent jet (symbols as in figure $14 a, b),(b)$ across a plume (symbols as in figure $13 b$ ).

contribution of the turbulent tracer flux has been generally neglected, being considered small in comparison to the mean advective tracer flux i.e. $\overline{w^{\prime} c^{\prime}} \ll \bar{w}_{\mathrm{c}} \bar{c}_{\mathrm{c}}$. If the initial jet Rhodamine $6 \mathrm{G}$ dye flux is $H_{0}=Q C_{0}$, then at a cross-section transverse to the jet axis downstream from the jet source, continuity implies that

$$
\begin{aligned}
H_{0}=Q C_{0} & =\int_{0}^{b(z)} \overline{u c} 2 \pi r \mathrm{~d} r \\
& =\int_{0}^{b(z)} \overline{u(c} 2 \pi r \mathrm{~d} r+\int_{0}^{b(z)} \overline{u^{\prime} c^{\prime}} 2 \pi r \mathrm{~d} r=H_{\mathrm{M}}+H_{\mathrm{T}}
\end{aligned}
$$

where $H_{\mathrm{M}}$ and $H_{\mathrm{T}}$ are the contributions to the tracer transport by the mean flow and the turbulence respectively, $b(z)$ is a boundary at a distance $z$ downstream from the jet origin beyond which no tracer is detected. In a two-dimensional buoyant jet Kotsovinos \& List (1977) report the ratio $H_{\mathrm{T}} / H_{0}$ to vary from about $6 \%$ in jets to $40 \%$ in plumes. Nakagome \& Hirata (1976) and George et al. (1977) found considerably lower values, with $H_{\mathrm{T}} / H_{0}=0.15$ in a round plume. Kotsovinos (1975) measured a normalized velocity-temperature correlation to be $\overline{w^{\prime} T^{\prime}} / \bar{w}_{\mathrm{c}} T_{\mathrm{c}}=0.25$, much higher than that found by Ramaprian \& Chandrasekhara (1983) who report 


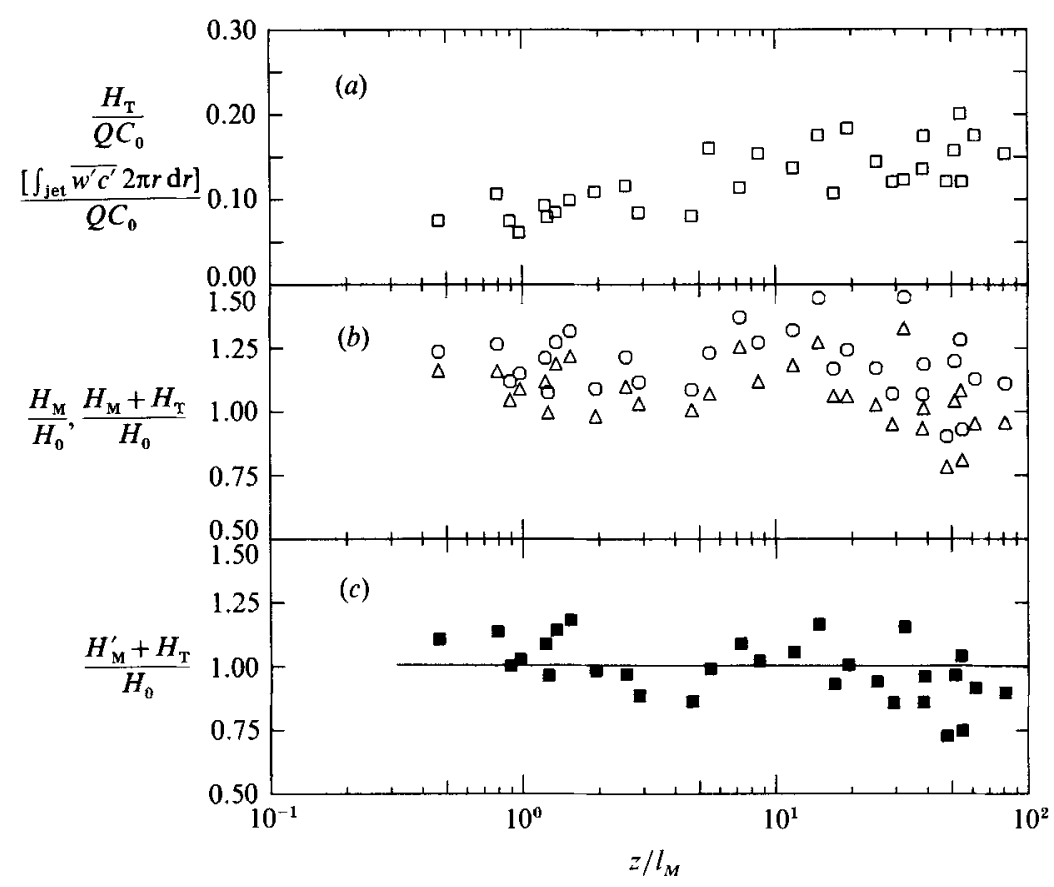

FIGURE 16. (a) Turbulent tracer flux in a buoyant jet normalized by the total flux of tracer at its origin $(\square)$. (b) Normalized calculated mean $(\triangle)$ and total $(O)$ tracer fluxes $H_{\mathrm{M}}$ and $H_{\mathrm{M}}+H_{\mathrm{T}}$ respectively in a buoyant jet. (c) Total normalized tracer flux calculated from the corrected concentrations ( $\square$ ).

0.05. Their laser-Doppler velocimeter, although far from noise free, was greatly improved over that employed by Kotsovinos (1975) and they had made special effort to see that the refractive-index fluctuations were minimal.

In the present investigation the total turbulent flux of Rhodamine $6 \mathrm{G}$ dye was computed directly from the radial distribution of $\overline{w^{\prime} c^{\prime}}$ for each experiment by integrating numerically the $\overline{w^{\prime} c^{\prime}}$ according to

$$
H_{\mathrm{T}}=\int_{0}^{b(z)} \overline{w^{\prime} c^{\prime}} 2 \pi r \mathrm{~d} r
$$

where $b(z)$ is defined to be the radial distance at which $\overline{w^{\prime} c^{\prime}}$ was zero. The amount of tracer transport by the turbulence, $H_{\mathrm{T}}$, normalized by the initial flux $H_{0}=Q C_{0}$ is plotted versus the dimensionless elevation $z / l_{M}$ in figure $16(a)$. In this figure it appears that the percentage of the tracer carried by the turbulence varied from $7-12 \%$ in jets $\left(z / l_{M}<1\right)$ up to $15-20 \%$ in plumes $\left(z / l_{M}>5\right)$. In consideration of the results in figure $16(a)$ it is important to discuss the closure $H_{0}=H_{\mathrm{M}}+H_{\mathrm{T}}$, where $H_{\mathrm{M}}$ is the advective mean tracer flux. In the present experiment, both $H_{\mathrm{M}}$ and $H_{\mathrm{M}}$ $+H_{\mathrm{T}}$ were found to exceed $H_{0}$ for almost all data points, as shown in figure $16(b)$. This means that either the advective or turbulent flux (or both) are overestimated, which indicates a systematic error. A comparison with the decay of the mean concentration on the flow axis measured by other authors with the results of the present investigation indicates that the absolute concentrations as measured by the LIF system are high. The results obtained by Papanicolaou \& List (1987) for 
temperature measurements in plumes support this hypothesis. They found that the decay of mean centreline temperature is given by

$$
\frac{S Q}{z M^{\frac{1}{2}}}= \begin{cases}0.165 & \text { for jets } \\ 0.090\left(\frac{z}{l_{M}}\right)^{\frac{2}{3}} & \text { for plumes }\end{cases}
$$

where $S=T_{0} / T_{\mathrm{c}}$ is the dilution ratio and $T_{0}$ and $T_{\mathrm{c}}$ are the initial jet and mean local centreline excess (i.e. above the ambient) temperatures. Using these temperature decay-rate constants in place of the concentration decay constants in the computation of $H_{M}^{\prime}$, the adjusted advective flux,

$$
H_{\mathrm{M}}^{\prime}=\int_{0}^{b(z)} \overline{w c_{1}} 2 \pi r \mathrm{~d} r \quad \text { with } \begin{cases}\bar{c}_{1}=\left(\frac{0.147}{0.165}\right) \bar{c}=0.890 \bar{c} \quad \text { (jets) } \\ \bar{c}_{1}=\left(\frac{0.070}{0.090}\right) \bar{c}=0.777 \bar{c} \quad \text { (plumes) }\end{cases}
$$

and keeping $H_{\mathrm{T}}$ as computed previously, the sum $H_{0}^{\prime}=H_{\mathrm{M}}^{\prime}+H_{\mathrm{T}}$ is found to take values close to $Q C_{0}$. This is shown in figure $16(\mathrm{c})$ where the sum of the adjusted advective mean tracer flux $H_{\mathrm{M}}^{\prime}$ and the unadjusted $H_{\mathrm{T}}$, normalized by $H_{0}=Q C_{0}$, has been plotted versus $z / I_{M}$, and takes an average value of about one.

A further check on the calculation can be obtained by determining the advective flux from the mean velocity and concentration or temperature profiles using the Gaussian-fitted distributions for tracer and velocity presented previously. These are $\bar{w}=\bar{w}_{\mathrm{c}} \exp \left[-\alpha(r / z)^{2}\right]$ with $\alpha$ equal to 93 and 90 in jets and plumes respectively, and $\bar{c}_{1}=\bar{c}_{\mathrm{c}} \exp \left[-\beta(r / z)^{2}\right]$ where $\beta$ takes the values 63 and 80 in jets and plumes respectively, where $\bar{c}_{1}$ is the corrected concentration. Using the velocity and corrected concentration decay along the flow axis, together with these exponential distributions one finds that in jets

$$
\left.H_{\mathrm{M}}=\int_{0}^{\infty} \overline{w c}_{1} 2 \pi r \mathrm{~d} r=\frac{\pi Q C_{0}}{(0.132)(0.165)(93+63)}=0.92 Q C_{0} \quad \text { (jets }\right)
$$

and in plumes

$$
H_{\mathrm{M}}=\int_{0}^{\infty} \overline{w c_{1}} 2 \pi r \mathrm{~d} r=\frac{\pi Q C_{0}}{(0.260)(0.090)(90+80)}=0.79 \mathrm{QC}_{0} \quad \text { (plumes). }
$$

This appears to confirm that the turbulent tracer flux varies from about $8 \%$ in jets to about $21 \%$ in plumes, which are approximately the same as the values determined by integrating numerically the $\overline{u^{\prime} c^{\prime}}$ profiles over the flow cross-section.

The adjustment in the mean concentration necessary to obtain a closure of tracer flux points to error involved in the measurements of the mean absolute concentration that was alluded to previously. It appears that the anomalously high absolute concentrations measured are attributable to an increase in the light intensity as seen by the laser-fluorescence system over that present during calibration of the system. It is not exactly clear why the experiments necessarily resulted in increased light scatter. However, it is believed that Rhodamine 6G dye absorbed on the organic micron-sized scattering particles used in the LDV system would result an increment in the fluorescence from the fluid probe volume. These particles were not present in the calibration solutions and filtering these particles to give better concentration measurements would make LDV impossible. In future it is clear that exactly the 


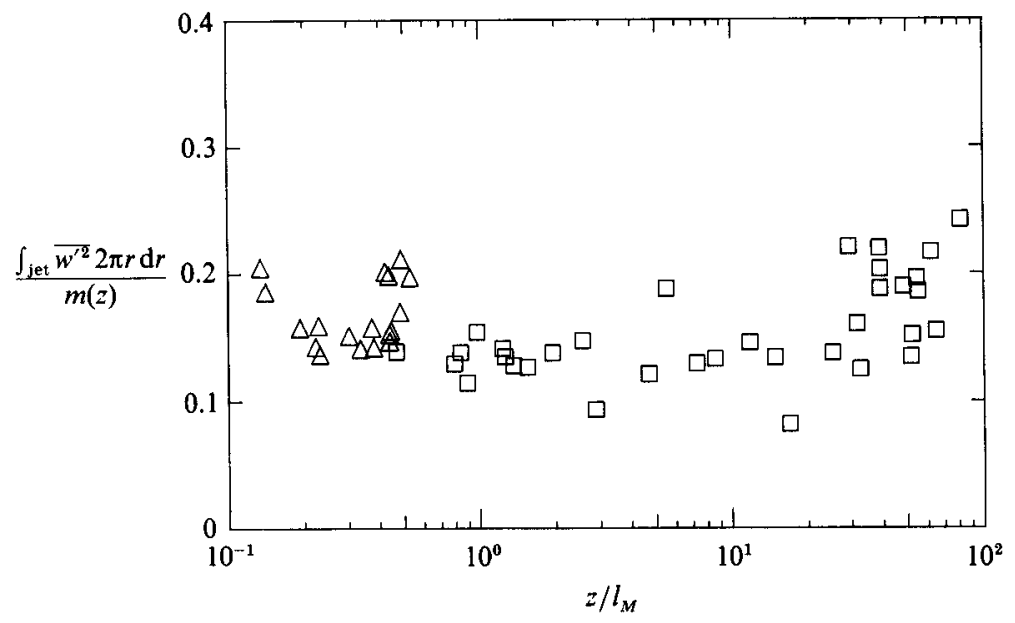

FIgURe 17. Turbulent momentum flux normalized by the mean momentum flux; $\square$, EXP; $\triangle$, PAP, VEL experiments.

same fluid should be used for calibration solutions as is used in the plumes. A more complete discussion of the errors involved in the joint use of LDV and LIF is given in the Appendix.

The turbulent momentum transport normalized by the advective transport, namely $m_{\mathrm{T}}(z) / m(z)$ is plotted in figure 17 as a function of $z / l_{M}$, where

$$
m_{\mathrm{T}}(z)=\int_{0}^{b(z)} \overline{w^{\prime 2}} 2 \pi r \mathrm{~d} r, \quad m(z)=\int_{0}^{b(z)} \bar{w}^{2} 2 \pi r \mathrm{~d} r
$$

These integrals were calculated numerically from the mean and r.m.s. velocity profiles. It is apparent that the turbulent-momentum-flux term $m_{\mathrm{T}}(z)$ is fairly constant with $z / l_{M}$ and approximately $15 \%$ of $m(z)$ for jets and plumes, in agreement with the estimated $14 \%$ obtained by List $(1982 b)$. The fact that the normalized turbulent transport fraction is constant is to be expected from the scaling arguments presented previously. It appears that the fraction of the jet momentum that is transported by the turbulence is significant and probably should not be neglected in the integral formulation of buoyant-jet theory that is frequently used (Muellenhof et al. 1985).

Other statistical properties of the Eulerian variables $w, u$ and $c$ can provide further information about the structure of turbulence. In figure $18(a, b)$ the local maximum (open symbols) and minimum (solid symbols) recorded axial velocity for jets and plumes (normalized by the mean centreline values) are plotted versus $r / z$. The profiles are quite self-similar and show that the maximum velocity is $1.65 \bar{w}_{\mathrm{c}}$ and occurs at the axis for both jets and plumes. The minimum observed velocities on the flow axis are positive and vary from $0.30 \bar{w}_{\mathrm{c}}$ in jets to $0.10 \bar{w}_{\mathrm{c}}-0.25 \bar{w}_{\mathrm{c}}$ in plumes. However, for radial distances beyond $r / z=0.10$ the minimum observed velocities become negative, with values varying from $-0.15 \bar{w}_{\mathrm{c}}$ in jets to $-0.20 \bar{w}_{\mathrm{c}}$ in plumes. This is in agreement with Lau, Morris \& Fischer (1979) who detected the existence of reverse flow in a jet at a radial distance $r / z=0.135$ using laser-Doppler anemometry. Given the similarity of the velocity profiles in jets and plumes the measured maximum and minimum concentrations (normalized by their mean 

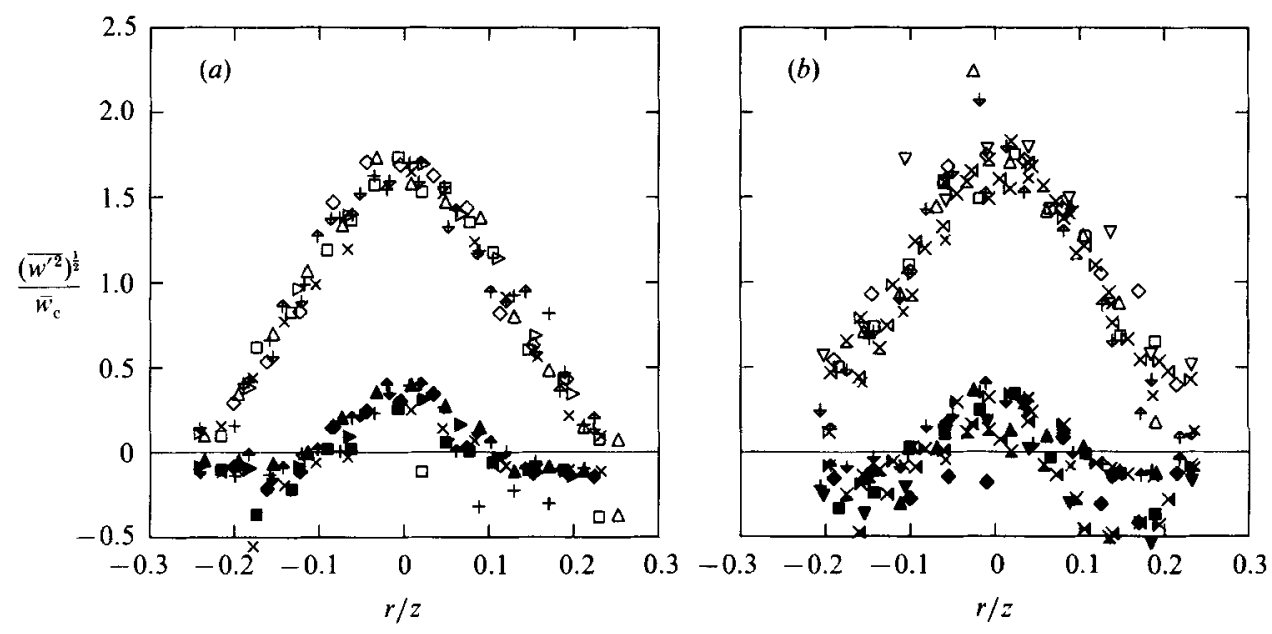

Figure 18. Profile of the maximum (open symbols) and the minimum (solid symbols) axial velocity $(a)$ across a turbulent jet, $(b)$ across a plume (symbols as in figures $13 b$ and $14 a$ ).
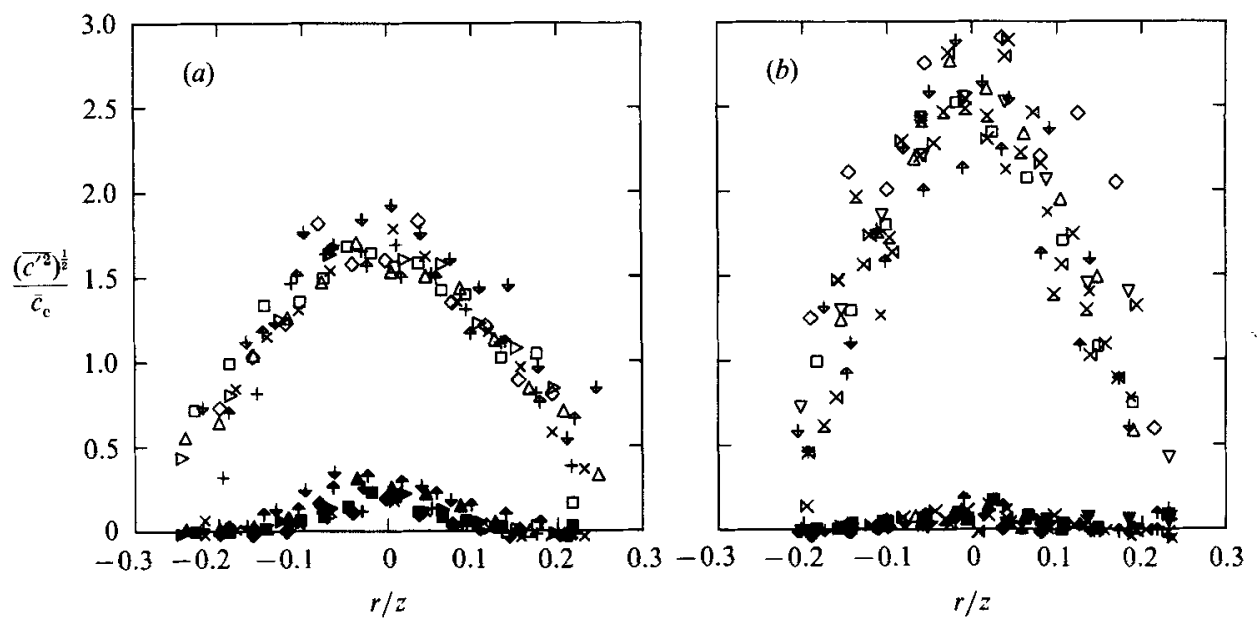

Figure 19. Profile of the maximum (open symbols) and the minimum (solid symbols) concentration $(a)$ across a turbulent jet, $(b)$ across a plume (symbols as in figures $13 b$ and $14 a$ ).

centreline values) that are plotted versus $r / z$ in figure $19(a, b)$ are surprising. More specifically the maximum measured concentration takes the value $1.65 \bar{c}_{\mathrm{c}}$ in jets and as high as $3.0 \bar{c}_{\mathrm{e}}$ in plumes. The minimum concentration on the axis is about $0.20 \bar{c}_{\mathrm{c}}$ in jets and zero beyond $r / z=0.10$. In a plume the minimum concentration appears to be close to zero everywhere, which means that in a plume, entrained unmixed ambient fluid reaches the flow axis even at substantial distances from the flow source. This is an extremely interesting topic that became the focus of another complete investigation and which will be reported separately. It is sufficient to note here that expansion of the LIF imaging system to include the entire span of flow has produced remarkable insight into the kinematics of the entrainment process.

The normalized third and fourth moments are plotted in figures 20 and 21 for jets and plumes. Figures $20(a-c)$ and $21(a-c)$ show that the skewness is zero around the axis for both jets and plumes and the flatness factors take the value 3.0 close to the 

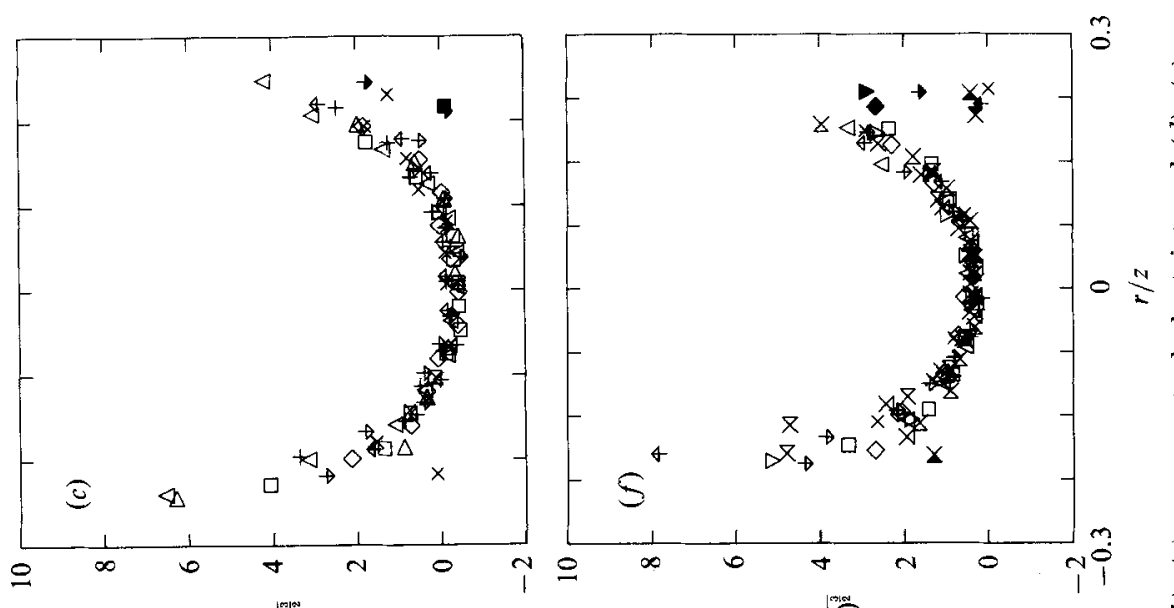

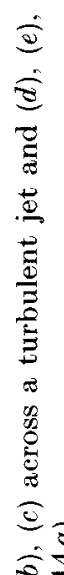

电
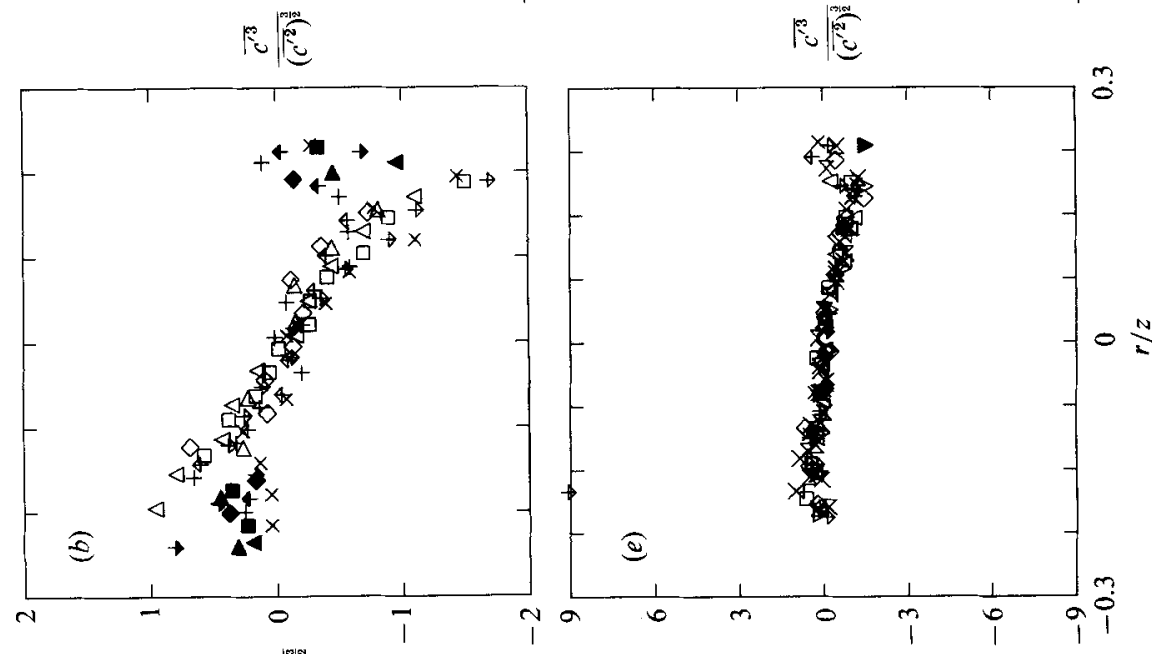

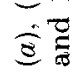

$\ddot{0}$

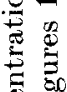

赵

$8 . \Xi$

(c)

要

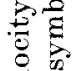

遂

丞

등

है

$F=\left.\right|^{m}$

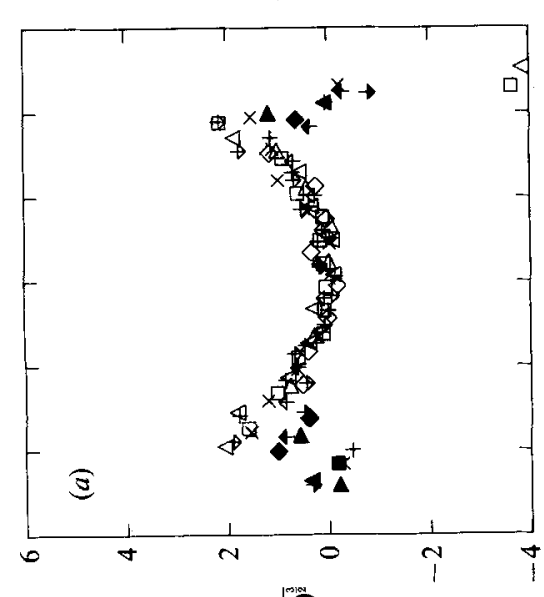

管

管

.

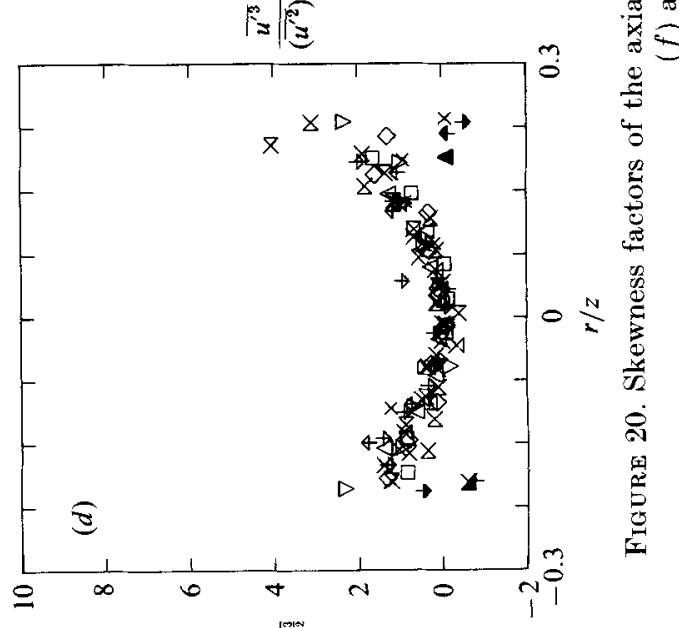

F 

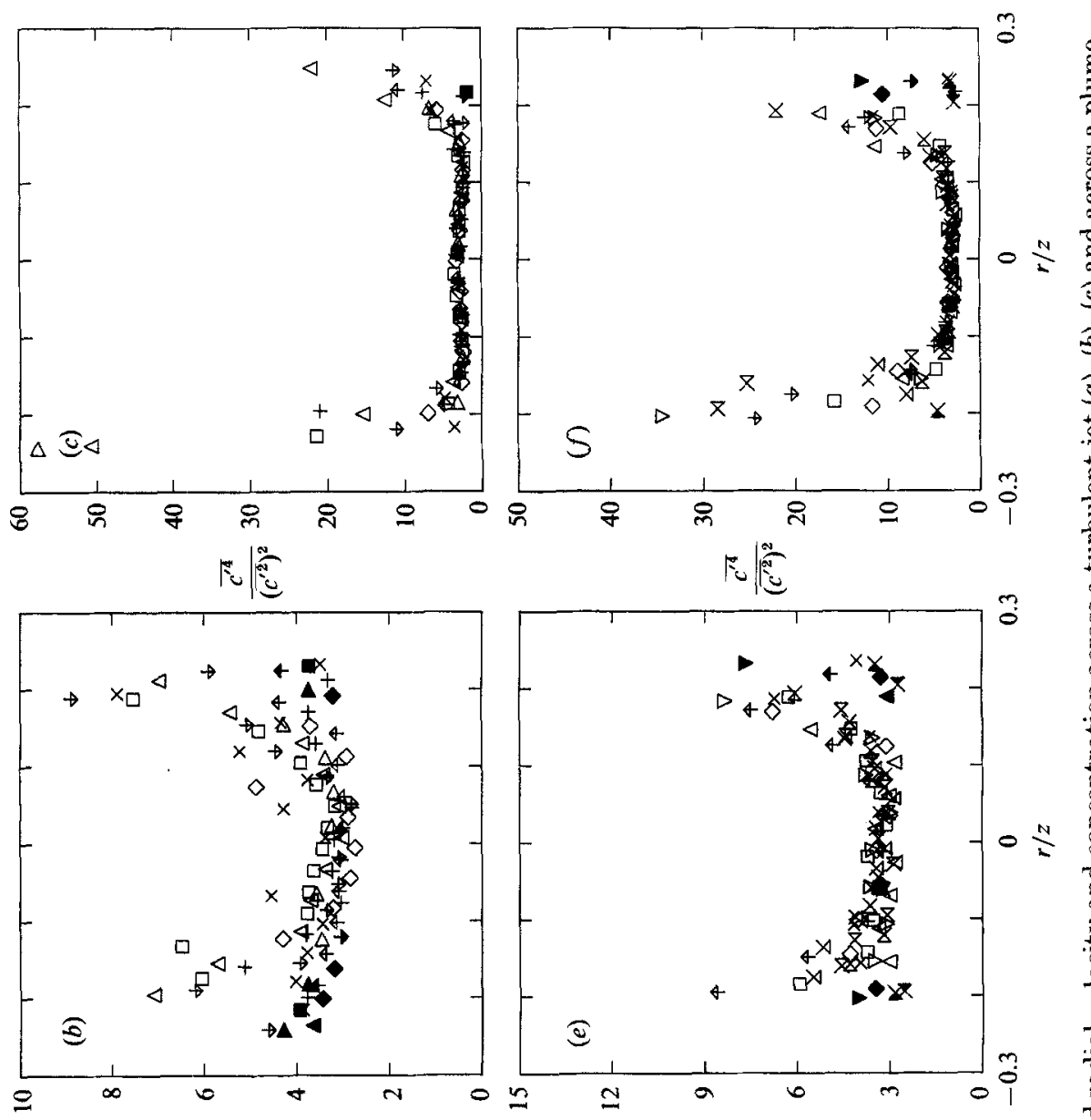

守

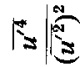
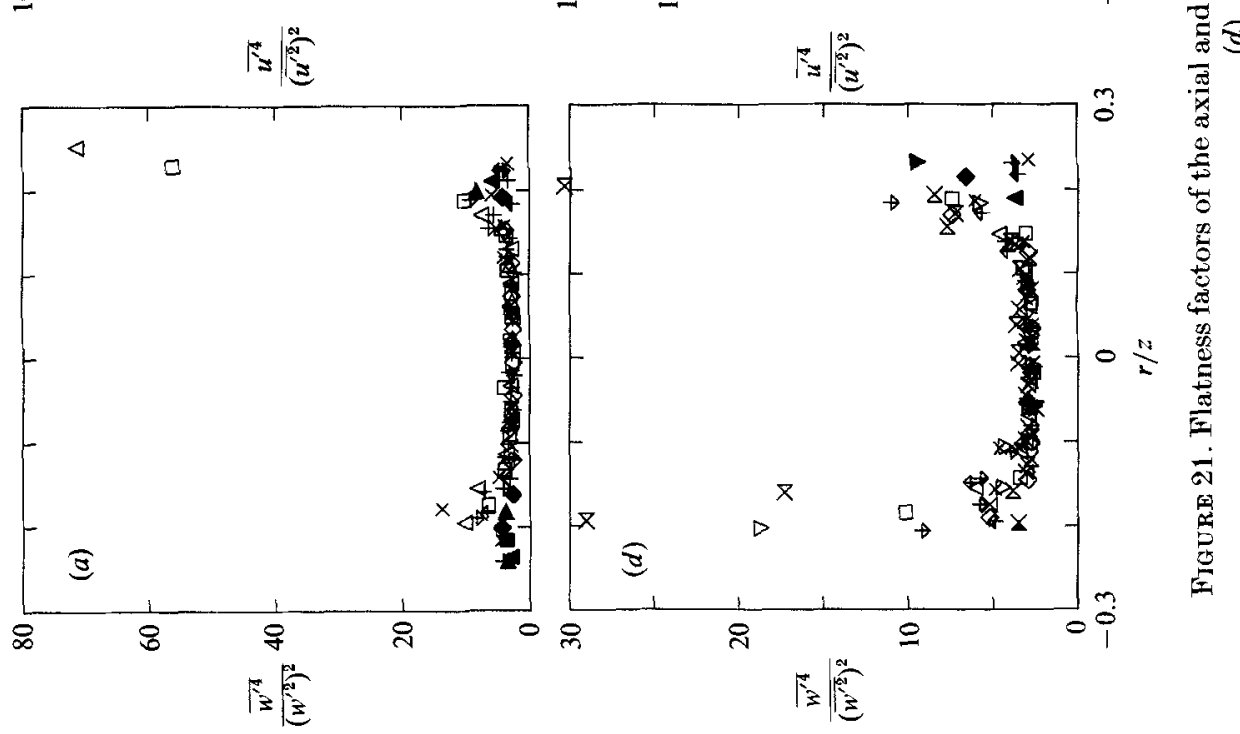


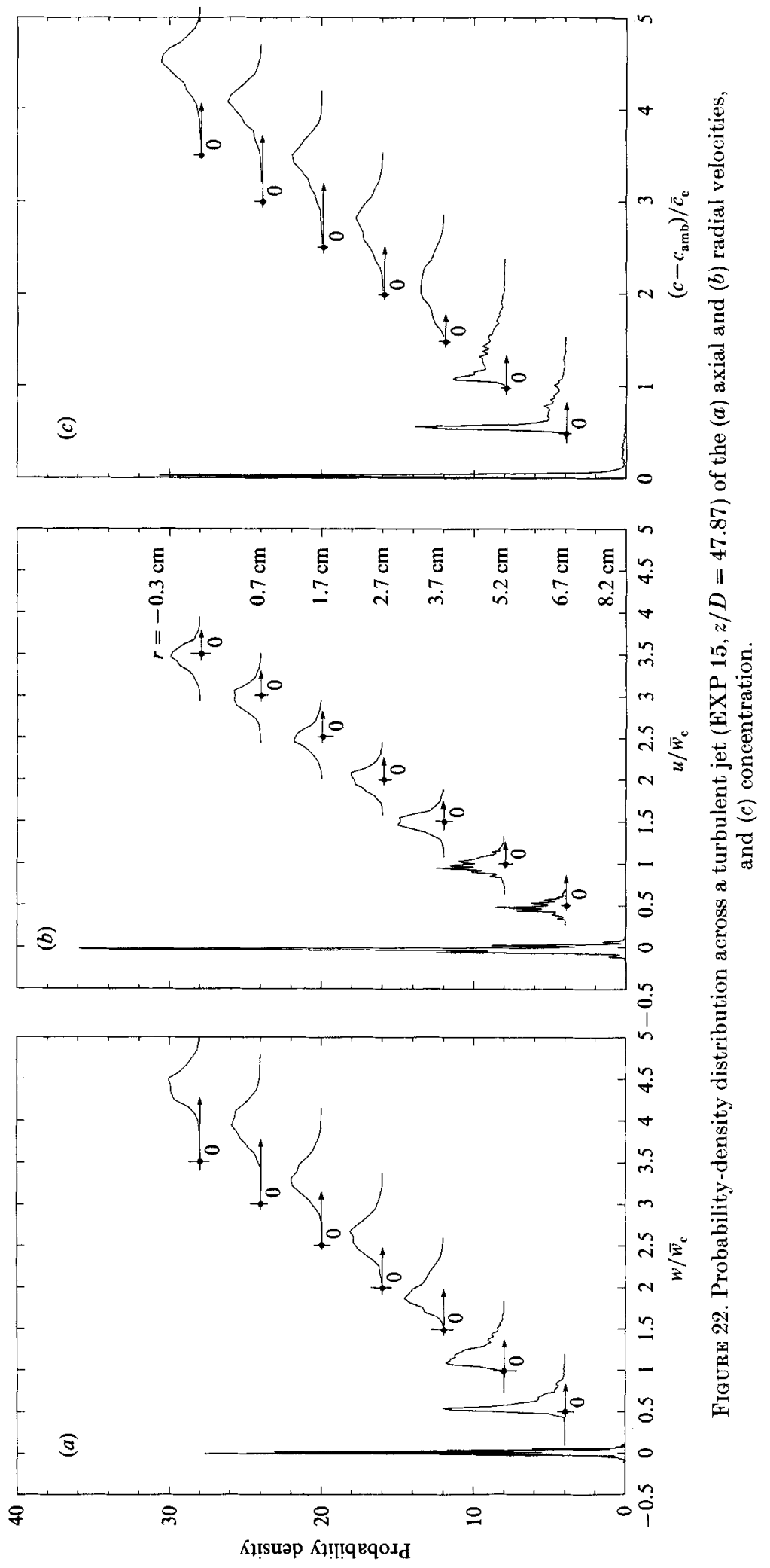




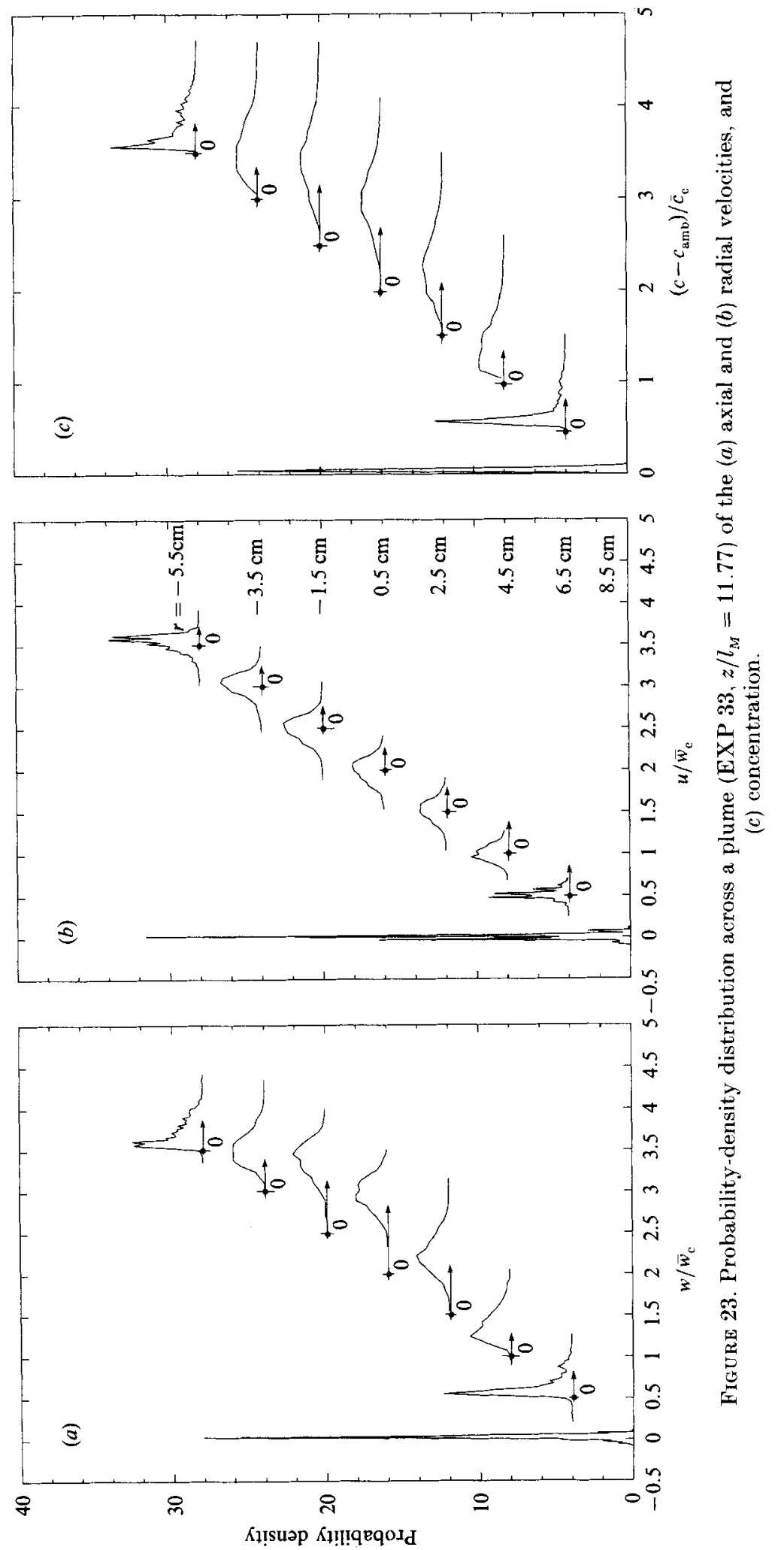


axis. The distribution of $w, u$ and $c$ near the axis of a buoyant jet appears to be closely Gaussian. The low values of the skewness and flatness factors for velocity at the jet boundary are due to the low signal-to-noise ratio at the jet boundary where the turbulence intensity is very small.

For a discrete time signal $\chi\left(t_{i}\right), i=1,2, \ldots N$, the probability $P(\chi)$ being in the interval $(\chi, \chi+\Delta \chi)$ is the ratio of the number of the discrete values $\chi\left(t_{i}\right)$ that occur in this interval divided by the total number of data points, if the record is adequately long. The probability density then is related to the probability as

$$
p(\chi)=\frac{P(\chi)}{\Delta \chi}
$$

and normalized by a characteristic value $\bar{\chi}_{\mathrm{C}}$

$$
\chi_{\mathrm{C}} p(\chi)=\frac{P(\chi)}{\Delta \chi / \bar{\chi}_{\mathrm{C}}}
$$

With this formulation we can plot the probability density of $w, u$ and $c$ in a dimensionless form. In figure $22(a-c)$ the normalized probability densities $p_{w} \bar{w}_{\mathrm{c}}$, $p_{u} \bar{w}_{\mathrm{c}}$ and $p_{c} \bar{c}_{\mathrm{c}}$ are plotted versus $w / \bar{w}_{\mathrm{c}}, u / \bar{w}_{\mathrm{c}}$ and $c / \bar{c}_{\mathrm{c}}$ respectively for a jet (EXP15) for various radial distances at $z / D=48$. The probability-density distribution of all three parameters $w, u$ and $c$ appears normal around the centreline, which agrees with the values of skewness and flatness factors 0.0 and 3.0 respectively, which were discussed above. The probability-density distributions peak at the jet boundary but near the jet boundary the distribution of the axial velocity indicates negative velocities $\left(w / \bar{w}_{\mathrm{c}}<0\right)$ as shown in figure $18(a, b)$. Clearly the rotational velocity within an eddy exceeds the net advective velocity. (The three peaks in the probability-density distribution for the radial velocity at the boundary is a result of the low signal-to-noise ratio rather than any resonant frequencies being apparent in the axial velocity time record.) Figure $23(a-c)$ shows the probability density distributions of $w, u$ and $c$ at various radial distances in a plume (EXP33) at an elevation $z / l_{M}=11.77(z / D=35)$. The results show a very significant probability that the local concentration within the plume will exceed the centreline mean value.

\section{Discussion and conclusions}

Non-intrusive optical experimental techniques have been employed to measure velocity and tracer concentration at points within turbulent buoyant jets. The sensitivity of this instrumentation has permitted experiments to be performed well into the asymptotic range of fully developed plume flows. Comparison of the results obtained in turbulent jet flows with results obtained previously by others has provided a measure of confidence in the techniques. The basic results of the study appear to confirm that the formulae used to describe asymptotic jet and plume flows, and developed from dimensional analysis, do provide a reasonable basis for prediction of time-averaged quantities such as velocity, concentration and volume flux. The study has also shown that the lengthscale $l_{M}$, defined by the specific momentum and buoyancy (density-anomaly) fluxes, does provide an excellent local definition of the relative degree of jet-like and plume-like character to the flow both for mean flows and r.m.s. quantities. This lengthscale, analogous in many ways to the Monin-Obukhov lengthscale used in atmospheric boundary-layer studies, when 
taken in conjunction with the distance from the flow source $z$ in the ratio $z / l_{M}$, essentially defines jets and plumes, with $z / l_{M}<1$ being jet-like and $z / l_{M}>5$ being established plumes.

Similarly a flux Richardson number can be defined for the flow, to parametrize the relative transfer rate of energy from available potential energy to kinetic energy. Stable plumes clearly have an equilibrium transfer rate in which the flux Richardson number becomes constant at a value of about 0.7 . The experimental results seem to resolve the question of the ratio of the width of the mean concentration and vertical velocity profiles to give a value of $\lambda=b_{w} / b_{c}=1.19$. The entrainment coefficient for a plume is found to be close to the $5 / 3$ for a jet predicted by List \& Imberger (1973) on the basis of perturbation analysis of the integral equations of motion.

The experimental results presented also appear to support quite strongly the hypothesis that the root-mean-square turbulent fluctuations in velocity and concentration in jets and plumes scale with the mean velocity and concentration. There is an abrupt change in the nature of the rate of decay of turbulent fluctuations that is defined by the distance from the flow origin normalized by the characteristic lengthscale $l_{M}$. In jets, the turbulent intensity of axial velocity fluctuations, as defined by the r.m.s. deviation from the mean, is fixed at about $25 \%$ of the timeaveraged mean velocity on the axis. The turbulent intensity of concentration fluctuations is fixed at about $22 \%$ of the time-averaged concentration on the axis, but at about $r / z=0.10$ this intensity increases to a maximum of $26 \%$. These results are, of course, well known and simply indicate that the peak turbulence level of the concentration of a tracer transported by the jet is associated with the maximum mean shear stress. In plumes, on the other hand, the maximum r.m.s. velocity and concentration fluctuations have a maximum of about $24 \%$ and $40 \%$ of the mean axial velocity component and concentration respectively, and the maxima are located on the plume axis. This is in congruence with the two turbulence production mechanisms being defined by the density/velocity correlation. Thus the distribution of density/velocity correlations becomes an important feature in the dynamics of plumes. Both jets and plumes display a broader mean concentration profile than mean velocity profile and a much wider r.m.s. concentration fluctuation profile than r.m.s. velocity profile. That the normalized mean velocity profile is narrower than the normalized mean concentration profile can be ascribed to the presence of negative velocities in the temporal record at points on the boundary of the turbulent flow. This argument does not, however, apply to the r.m.s. fluctuations. There are two hypotheses to explain this feature of the motion. For the r.m.s. velocity fluctuations the pressure-work term in the turbulent kinetic-energy balance could provide a relaxation mechanism that does not exist for the concentration fluctuations. Furthermore, the molecular diffusivity for concentration is approximately two orders of magnitude less than that for vorticity, so that it is to be expected that concentration gradients will persist much longer than vorticity gradients. However, experiments in which temperature is used as the tracer contaminant also show a broader r.m.s. tracer fluctuation profile, which suggests that the pressure--work relaxation term may be the significant factor. Until a method is developed to determine this term directly this will probably have to remain a conjecture.

The measurements of turbulent flux of tracer do not support the very high levels found by Kotsovinos \& List (1977). The explanation for this probably lies in the contribution to velocity measurements made by the refractive-index fluctuations in Kotsovinos ' experiments. His experiments used a temperature increment as a tracer and this would result in refractive-index fluctuations of about 0.0001 for a $1{ }^{\circ} \mathrm{C}$ 
temperature increment. Practical experience with modern LDV systems indicates that such fluctuations create enough beam wobble to create problems with data collection for beam paths in excess of $50 \mathrm{~cm}$.

In the experiments performed here the turbulent tracer flux was measured directly with very low salinity concentrations as the tracer. The flux was evaluated both directly from measurements of fluctuating velocity and concentration, and indirectly by differencing the initial total flux and the mean advective flux computed from the mean velocity and concentration profiles. These results, although containing some inherent noise, indicate an increase in the turbulent flux of from $7-12 \%$ in jets to $15-20 \%$ in plumes. It would seem that this is more reasonable than the $40 \%$ indicated by Kotsovinos although it is larger than that measured by Nakagome \& Hirata (1976) and George et al. (1977), both of whom conducted experiments in what was far from a fully developed plume. This result implies that integral models based on an entrainment hypothesis, such as that of Morton, Taylor \& Turner (1956), and all the subsequent variants applied to cross-flow, stratification and so forth, should perhaps contain some adjustment for this flux contribution. Similarly, the turbulent flux of momentum appears to be approximately $15 \%$ of the advective momentum flux in plumes.

The experimental results obtained also indicate that the emphasis placed on timeaveraged quantities by current computer models may require reconsideration, especially where maximum predicted concentrations are concerned. Computer models of buoyant discharges that are currently in use by environmental control agencies concerned with air and water pollution often predict a flux-weighted average dilution of a discharged tracer, which of course increases with distance from the flow source. Measurements obtained with reasonably high-resolution nonintrusive instrumentation, such as the laser-induced-fluorescence system used here, show that flux-weighted average dilutions may give concentrations of tracer that are as much as three times more dilute than actual peak concentrations. For evaluation of toxic and flammable gas and liquid spills such a factor of three may be extremely important in determining safe limits for discharged materials.

In summary, the experimental work described here provides a basis for evaluating the reliability of integral and dimensional methods of analysis for buoyant flows, as well as the popular turbulence closure models. The fact that root-mean-square fluctuations are seen to be self-similar and scale as time-averaged mean values should enable these terms to be included easily as corrections to existing models.

The support of the National Science Foundation through Grant Nos. CEE8117272A01 and MSM84-12641A01 is gratefully acknowledged.

\section{Appendix. The simultaneous measurement of fluid velocity and tracer concentration}

\section{A.1. Introduction}

The study of round (three-dimensional) buoyant jets and their turbulence properties are of importance both in engineering applications and in the understanding of the structure of turbulent shear flows. Prior studies of momentumdriven jets in the region beyond 50 jet diameters downstream from the nozzle appear to provide a comprehensive description of the flow. However, measurements of velocity and concentration in the flow field of buoyancy-driven plumes are limited to 
about 20 plume diameters downstream from the flow origin, primarily because of the limited applicability of the conventional probe-based techniques in rapidly diluting plume flows.

Early investigators of heated jets, such as Corrsin (1943), Corrsin \& Uberoi (1949) and Hinze \& van der Hegge Zijnen (1949) used hot-wire anemometers, total-head tubes, thermocouples and sample analysers for velocity and temperature (or concentration) measurement. Rosenweig et al. (1961) measured concentrations in an air jet utilizing smoke-scattered light. Forstall \& Gaylord (1955) performed conductivity measurements along the centreline of a salt-water jet which discharged in a volume of fresh water. Using a light-scattering technique Becker et al. (1967) measured concentrations in a jet traced by oil smoke at up to 85 jet diameters. Antonia et al. (1975) measured simultaneously two velocity components and the temperature conditionally in a heated jet within a coflowing stream, using $\mathrm{X}$-wire anemometers 59 jet diameters from the jet origin. Kotsovinos (1977) combined a onedimensional laser-Doppler anemometer with a fast-response thermistor probe to measure simultaneously the longitudinal velocity and temperature in a twodimensional (plane) heated jet. He reported that the heat flux transported by the turbulence varied from about $10 \%$ of the total in a jet to almost $40 \%$ of the total in a plume. George et al. (1977) and Nakagome \& Hirata (1976) reported substantially smaller turbulent heat flux than reported by Kotsovinos (1977) at up to 15 plume diameters. Zimin \& Frik (1977) measured the temperature distribution in a heated jet by observing the changes in refractive index of the fluid with temperature variation. A very interesting experiment was performed by Birch et al. (1978) who measured concentrations ir a round jet using laser Raman spectroscopy.

Owen (1976) reported the first successful application of combined laser anemometry and induced fluorescence in the measurement of the initial mixing region of two confined coaxial jets. Johnson \& Bennett (1981) also performed this experiment. However, neither the sources of possible error and limitations of the technique, nor the closure in tracer conservation (as a measure of the error involved in the measurement) were discussed by these authors. Papanicolaou \& List (1984) applied the technique to be discussed here successfully in a buoyant jet, and obtained closure of the tracer conservation equation (i.e. the sum of tracer transported by the mean flow and turbulence at any cross-section of the jet was found to be equal to the amount of tracer injected at the jet nozzle).

As previously noted intrusive probe-based techniques make temperature or concentration measurements in the far field of buoyant jets very difficult. Furthermore, the simultaneous velocity and concentration (temperature) measurements at the same point in the flow are almost impossible because of probe interference. For example, Kotsovinos \& List (1977) who measured simultaneously one velocity component and the temperature at two points located $2.5 \mathrm{~mm}$ apart, assumed that the spatial velocity- temperature correlation was perfect. In this study we describe a technique in which four laser beams are used to measure simultaneously two components of velocity and tracer concentration. A combination of two different colours (frequencies) of laser-light allows the simultaneous measurement at the same point in a turbulent shear flow without interference. This is accomplished with a tworeference-beam laser-Doppler velocimeter (LDV) combined with a recently implemented laser-induced-fluorescence (LIF) device. The simultaneous measurement of the axial $w$ and the radial $u$ velocity components and concentration $c$ of Rhodamine $6 \mathrm{G}$ dye within a round turbulent buoyant jet has been accomplished. In the following we describe the principles of the technique and its implementation and application. 


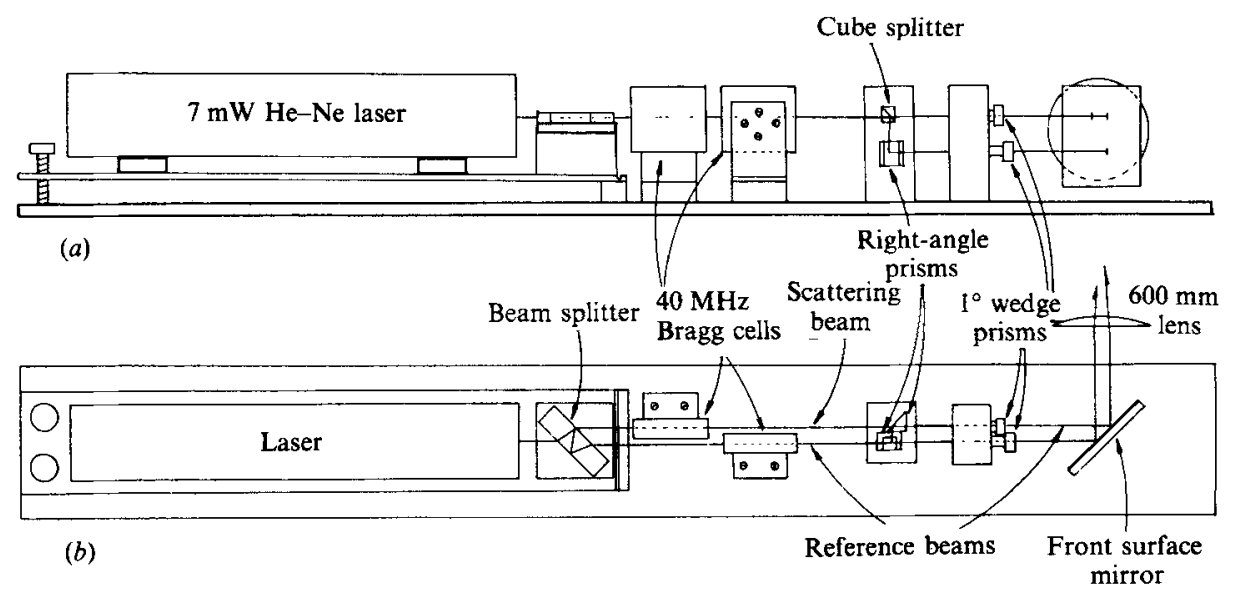

Figure 24. Optical configuration for the laser-Doppler velocimeter (LDV): (a) elevation, (b) plan.

\section{A.2. The experimental set-up}

A turbulent buoyant jet was created in a $3.3 \mathrm{~m}$ deep and $1.15 \mathrm{~m}$ square tank of water. An instrument carriage was attached to the tank to allow three-axis movement of the four laser beams used in the experiment. Specific details of these instruments are as follows.

\section{A.2.1. The laser-Doppler velocimeter}

The vertical (axial) and horizontal (radial) velocity components $w$ and $v$ were measured simultaneously at the same point of the flow using a two-reference-beam laser-Doppler velocimeter (LDV). For a complete description of the basis of the technique, see Watrasiewicz \& Rudd (1976), Drain (1980), Durst, Melling \& Whitelaw (1976) and Papanicolaou (1984). The scatter and reference beams were shifted relatively at about $70 \mathrm{kHz}$ by means of two $40 \mathrm{MHz}$ Bragg cells excited by oscillating crystals. The parallel beams were focused at the same point near the middle of the tank by a $600 \mathrm{~mm}$ focal-length converging lens. All the optics and the laser were firmly attached to a $1.27 \mathrm{~cm}$ thick jig plate, which was bolted to the instrument platform. The beam location in space, and the beam crossing point, were determined using a theodolite. The optical layout of the laser-Doppler velocimeter is shown in figure 24 .

\section{A.2.2. The laser-induced-fluorescence system}

According to Drexhage (1976) a water solution of a Rhodamine 6G dye excited by a monochromatic source of light with wavelength $\lambda_{\text {in }}=514 \mathrm{~nm}$, emits light (fluoresces) at a wavelength around $\lambda_{\mathrm{em}}=570 \mathrm{~nm}$. This laser-induced-fluorescence (LIF) principle is used in a concentration measuring device as shown schematically in figure 25. A collimated laser beam of intensity $I$ and a wavelength $\lambda_{\text {in }}=514 \mathrm{~nm}$ excites a Rhodamine 6G dye water solution which fluoresces at a wavelength $\lambda_{\text {em }}=$ $570 \mathrm{~nm}$. A photodetector located at the point A' 'sees' the point A of the beam through a converging lens $L$. The light which reaches the photodetector through an aperture is a mixture of the fluorescent light at about $570 \mathrm{~nm}$, and $514 \mathrm{~nm}$ light scattered from possible foreign particles suspended in the water. If the light incident to the photodetector is high-pass filtered $(\lambda>530 \mathrm{~nm})$, then the photodetector will 


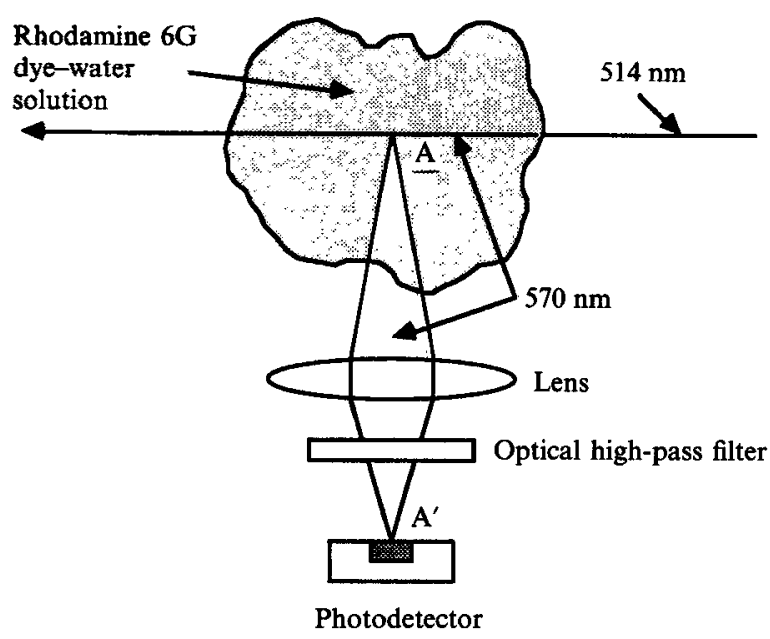

Figure 25. Principle of the laser-induced fluorescence (LIF) of a Rhodamine 6G dye-water solution.

only detect the fluorescent light. The output current intensity of the photodetector is 'proportional' to the intensity of the fluorescence from the point $\mathrm{A}$, which means proportional either to the Rhodamine $6 \mathrm{G}$ dye concentration at that point, or to the intensity of the laser beam exciting the Rhodamine 6G dye solution. A fixed laser light intensity of about $800 \mathrm{~mW}$ with a collimated beam of $1 / \mathrm{e}^{2}$-diameter $0.4 \mathrm{~mm}$, provided a linear current output from the photodetector for Rhodamine $6 \mathrm{G}$ dye concentrations varying between 1 and 40 p.p.b. The minimum dye concentration that the photodetector could detect was approximately 0.4 p.p.b. for a laser light intensity of around $800 \mathrm{~mW}$. The laser was operated at the light-stabilized mode because it provided a constant light intensity after the laser was warmed up. The stability of the laser light intensity was also verified from long concentration records at a specific point in the tank that was maintained at uniform concentration. The recorded signal variance of about 0.05 p.p.b. was a result of the electronically induced noise rather than variation in the laser light intensity. The noise appeared as a peak at $60 \mathrm{~Hz}$ in the power spectrum of the concentration fluctuations. The LIF laser beam collimated by a converging $750 \mathrm{~mm}$ lens crossed the intersection of the two reference beams and the scattering beam used in velocimetry.

\section{A.2.3. The receiving optics - data aquisition system}

The optical layout of the combined LDV and LIF is shown in figure 26. Two fastresponse silicon photodiodes collected the heterodyning light from the two LDV reference beams and the LDV scattering beam. The photodiodes were masked by $0.5 \mathrm{~mm}$ pinholes and two $125 \mathrm{~mm}$ converging lenses were placed in front of the pinholes at a distance equal to their focal length. The reference beams deflected by two front-surface mirrors were directed into the pinholes through the centre of the lenses. Hence small changes in the refractive index (due to density variations), which made the reference beams wobble, would not affect the velocimetry except through the induced movement of the beam crossing. The signal was normally interrupted about $50 \%$ of the time and use of the lenses provided an almost uninterrupted signal, since the reference beams crossed continuously the focal point of the lenses. A third photodiode was used to collect the light emitted from the intersection of all four 


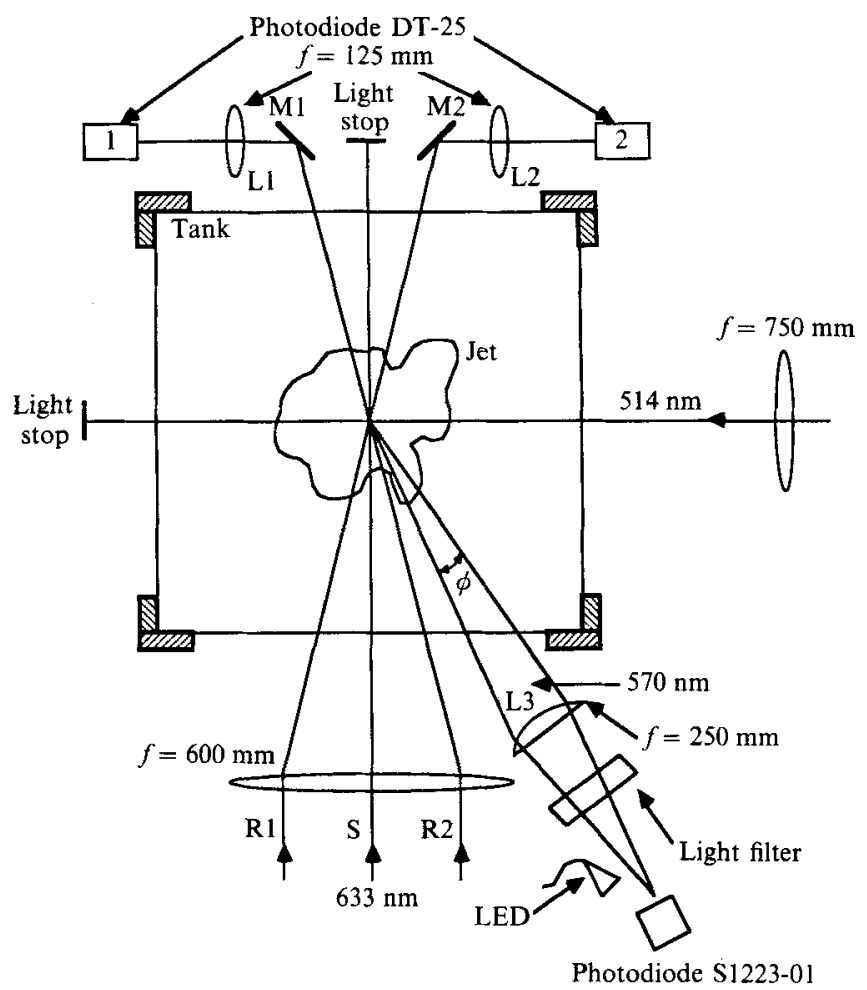

FIgURE 26. Plan-view configuration of the receiving optics for the LDV and LIF measuring systems.

beams in a solid angle $\phi$ through the $250 \mathrm{~mm}$ converging lens. A small light-emitting diode (LED) excited this photodiode and moved its operational point into the linear response regime. This light source did not affect the concentration measurement since it was taken into account during the calibration. This photodiode was located at the same side of the tank as the velocimeter transmitting optics, so that any bias to the signal from the forward scattering of the laser light used in velocimetry was avoided. The back scattering from the LDV had no effect in the LIF signal. A highpass optical filter was used in order to avoid effects from the scattered light at a wavelength of $514 \mathrm{~nm}$. A vertical slit $0.152 \mathrm{~mm}$ wide and $1.5 \mathrm{~mm}$ long was used instead of a pinhole in front of the LIF photodiode, so that the horizontal line image was always detected. The image moved up and down with possible changes in the refractive index in the flow field and because of possible structural imperfections of the instrumentation carriage as it was moved. The probe volume that was imaged on the photodiode was calculated and found to be a cylinder of diameter $0.4 \mathrm{~mm}$ and length $0.4 \mathrm{~mm}$.

The current signal from the photodiodes was converted into a voltage. The preamplified LDV signal was amplified and band-pass filtered with a centre frequency set near the expected Doppler frequency. The signal was then passed through a dual counter-based processor described in detail in Gartrell (1978) and Papanicolaou (1984) and was stored in binary form for subsequent analysis. The fluorescence signal was also preamplified and converted into a voltage, then amplified to a voltage range of $\pm \mathbf{5 . 0}$ volts. The amplified signal was low-pass filtered and stored in digital form together with the LDV signal. 


\section{A.2.4. Advantages and limitations of the technique}

The main objective of this work was to determine the turbulence characteristics in round jets and plumes by measuring simultaneously two velocity components and the concentration of a tracer and to obtain estimates of the tracer and momentum fluxes transported by the jet turbulence. Use of laser-Doppler velocimetry combined with a thermistor probe set close to the focal volume of the velocimeter (see Kotsovinos \& List 1977 and Gartrell 1979) did not satisfy our experimental needs. Prior temperature measurements using fast-response thermistor probes (Papanicolaou \& List 1987) showed that the resolution that one could obtain for the time average and turbulent temperatures was poor beyond 40 jet or plume diameters because of the rapid dilution within the plume. This problem did not occur in Kotsovinos' (1975) plane-flow studies where he was able to measure temperature profiles up to 90 jet lengths and 40 plume lengths downstream. The reason for this is that the mean temperature in a round jet decays much faster than in plane jets. More specifically, $T \approx z^{-\frac{1}{2}}$ and $T \approx 1 / z$ in plain jets and plumes respectively, whereas $T \approx 1 / z$ and $T \approx z^{-\frac{5}{3}}$ in round jets and plumes. In summary, the use of an optical technique versus an intrusive probe-based technique is significantly more advantageous in the present experiment for the following reasons. (i) Probe-based techniques, such as thermometry or conductivity measurement do not give adequate resolution beyond 40 jet or plume diameters in round buoyant jets, which is the region where Wygnanski \& Fiedler (1969) and Birch et al. (1978) showed that a jet is fully developed and self-similarity is obtained. In the fluorescence studies here the use of high initial jet Rhodamine $6 \mathrm{G}$ concentrations can still provide a mean concentration of 5-10 units (p.p.b.) beyond 100 jet diameters downstream from the origin and an initial jet concentration of about 200 p.p.b. does not affect the density of the jet fluid. Therefore an initially jet-like flow will still behave as a jet for $z / D>100$, whereas the use of salinity or temperature as a tracer will convert the jet into a plume at this distance from the flow origin. (ii) Thermistor probes can give a certain error at the jet boundaries where reverse flow appears, the reason being that the fluid attached to the glass bead of the probe cannot be removed properly resulting in a bias in the temperature measurement. The induced-fluorescence technique is independent of the direction of the flow. (iii) Optical techniques do not disturb the flow as do intrusive probe-based techniques. Calculations have shown that the dissipated heat from the $800 \mathrm{~mW}$ laser beam is negligible and does not affect the flow. (iv) When the temperature probe is $2.5 \mathrm{~mm}$ from the focal volume of the velocimeter, as used by Kotsovinos (1975), a bias is possible due to $u-T$ spatial correlations.

\section{A.2.5. Tank, design and operation; instrument carriage}

Four corner posts, constructed from rectangular steel tubing cross-sections and welded together, support four $2.54 \mathrm{~cm}$ thick tempered glass panels with dimensions $1.20 \mathrm{~m}$ wide and $1.85 \mathrm{~m}$ high (upper tank). A square overflow is placed $10 \mathrm{~cm}$ below the top of the tank. It is constructed from a $12.7 \mathrm{~cm}$ wide aluminium $\mathrm{U}$-section and has a knife edge towards the glass to enable uniform surface drainage of excess water. Four precision cylindrical rails $2.54 \mathrm{~cm}$ in diameter are bolted in a vertical position to the four corner posts and are used as supports and guides for the instrument carriage. This upper tank is bolted to a lower tank constructed from five $0.64 \mathrm{~cm}$ thick steel plates welded together (four on each side and one on the bottom). The entire tank is bolted to the floor. Four air injectors are placed in the bottom of the 
tank walls and form a mixing system to provide homogeneity in the density and concentration of the tank fluid before each experiment.

The transmitting and receiving optics of both the laser-Doppler velocimeter and the laser-induced-fluorescence system, are mounted firmly on a counter-weighted instrument carriage. It is attached to the tank so as to provide both vertical and lateral movement for the instrumentation. The carriage consists of two parts : $(a)$ a main frame (rigid box) which is attached to the tank; and $(b)$ a platform on which the instrumentation is mounted that can slide on two shaft assemblies attached to the main frame. The main frame is a rigid box constructed from $10.2 \times 10.2 \times 1.3 \mathrm{~cm}$ square aluminium tubing and a $10.2 \mathrm{~cm}$ wide aluminium $U$-section to provide rigidity. Eight cylindrical bearings, two on each corner of the box, mount the frame to the four $2.5 \mathrm{~cm}$ precision shaft assemblies bolted on the corner posts and allow it to move vertically. The carriage was counterweighted by four weights connected to cables allowing $1.50 \mathrm{~m}$ of vertical motion of the carriage. For additional details on the tank and instrumentation carriage see Papanicolaou (1984).

\section{A.3. The experimental procedure}

\section{A.3.1. Calibration and errors in velocimetry}

Calibration of the laser-Doppler velocimeter was obtained electronically and was found to be stable for extended periods of time. A frequency generator fed the counter with sinusoidal waves at known frequencies $\left(f_{i}\right)$. The analog output of the processor was sent and recorded by the computer in digital (binary) form $\left(V_{i}\right)$. A least-square fifth-order polynomial

$$
f_{w, u}=\frac{A}{V^{2}}+\frac{B}{V}+C+D V+E V^{2}
$$

fit to the data pairs $\left(f_{i}, V_{i}\right)$ accounted for possible nonlinearity. The Doppler frequency $f_{\mathrm{D}}$ then was calculated from the measured frequencies $f_{w, u}$ as $f_{\mathrm{D}}=f_{w, u}-F$, where $F$ is the electronically measured frequency shift between the reference and scattering beams. The various sources of error inherent in the velocimetry and the error estimates are discussed in the following paragraphs.

(i) Large scattering particles did not cause significant measurement error according to Gartrell (1979), since no particles larger than $5 \mu \mathrm{m}$ exist in the laboratory water and hence are sufficiently small that they follow the fluid trajectories.

(ii) Counter and data-digitization errors are due to the inability of the counter system to distinguish two signals of slightly different frequency. For details see Gartrell $(1978 ; 1979)$ and Papanicolaou (1984). The combined error due to digitization and the counter was measured as follows. A sinuosoidal wave of frequency $f=69.547 \mathrm{kHz}$ was fed in the processor (counter) and the output was sampled for $10 \mathrm{~s} \mathrm{(24-30} \mathrm{Doppler} \mathrm{periods} \mathrm{per} \mathrm{point)} \mathrm{at} \mathrm{a} \mathrm{rate} \mathrm{of} 50 \mathrm{~Hz}$ by the A/D converter and recorded by the computer in digital form. The calculated mean and r.m.s. values for the two velocity components were calculated to be $\bar{w}=-0.245 \mathrm{~cm} / \mathrm{s}$ $\left(\overline{u^{\prime 2}}\right)^{\frac{1}{2}}=0.017 \mathrm{~cm} / \mathrm{s}, \bar{u}=0.972 \mathrm{~cm} / \mathrm{s}$ and $\left(\overline{u^{\prime 2}}\right)^{\frac{1}{2}}=0.040 \mathrm{~cm} / \mathrm{s}$. Assuming that the absolute maximum error is twice the standard deviation, it is obvious then that the maximum absolute error was of the order of \pm 0.04 and $\pm 0.08 \mathrm{~cm} / \mathrm{s}$ for $w$ and $u$ velocities respectively.

(iii) It was desirable to have a 'continuous' velocity signal, therefore the threshold level for the Doppler bursts was lowered to near the noise level of the photodiodes 
and this introduced error into the system. Furthermore, the existence of many particles in the probe volume introduced some noise in the velocimetry. The combined error from all the previously described error sources (i), (ii) and (iii) was measured as follows. With the threshold level close to the photodetector noise level and with no flow in the tank the processor was fed with the output from the photodiodes. From the recorded digital signal the mean and r.m.s. values were calculated to be $\bar{w}=\bar{u}=0 \mathrm{~cm} / \mathrm{s}$ (as expected) and $\overline{\left(w^{\prime 2}\right)^{\frac{1}{2}}}=0.087 \mathrm{~cm} / \mathrm{s}$ and $\left(\overline{u^{\prime 2}}\right)^{\frac{1}{2}}=$ $0.156 \mathrm{~cm} / \mathrm{s}$ respectively. Therefore the maximum combined absolute error in the velocities due to the processor, data aquisition system, low threshold level for the Doppler burst detection, and multiparticle scattering from the beam-crossing did not exceed \pm 0.15 and $\pm 0.312 \mathrm{~cm} / \mathrm{s}$ for the axial and radial velocities respectively. The above-measured r.m.s. values of $w$ and $u$ are the maximum noise levels of the output signal of the system to the computer.

(iv) The maximum deflection of the glass panels occurred at $\frac{2}{3}$ the depth from the water surface and it was calculated and measured to be $1.30 \mathrm{~mm}$. The error in the beam-crossing location was calculated and measured to be $\pm 0.2 \mathrm{~mm}$ in the radial $(r)$ and $\pm 0.5 \mathrm{~mm}$ in the axial $(z)$ direction. The laser beam used for the induced fluorescence was affected by the glass deflection only in the vertical direction since it was only able to move on a vertical plane through the middle of the tank. Its relative displacement with respect to the centre of the probe volume for velocimetry was zero in the vertical $(z)$ direction and at most $\pm 0.2 \mathrm{~mm}$ in the radial $(r)$ direction.

(v) A very important source of error in the measurement of the velocity is due to the changes of refractive index of the fluid resulting from the density fluctuations created by variation in the tracer concentration. The resulting beam wobble can introduce noise to the velocity signal as $(a)$ fictitious turbulence due to the movement of the beam-crossing volume and $(b)$ discontinuities in the Doppler bursts received by the photodetector when the reference beam does not mix with the scattered light on the pinhole. To avoid the beam-wobble problem McDougall (1979) proposed matching the refractive indices of the jet and ambient fluid. He used two solutes to produce density differences which would avoid double-diffusion convection (saltfinger type) but still match refractive indices. McDougall's (1979) technique was successfully applied by the authors using sodium chloride and sugar-water solutions for the ambient and the jet fluids respectively. Use of reasonable amounts of salt and sugar resulted in an initial density difference of 2-3 $\sigma$-units. However, in order to reach the fully developed plume regime with the present experimental set-up, very large concentrations of salt and sugar would be needed to obtain initial density differences of 10-20 $\sigma$-units. On the other hand use of salt-water solutions for the jet, and fresh water for the ambient fluid gives an initial density difference of about 20 $\sigma$-units with a low salt concentration in the highly diluted plume region of flow.

The velocity measuring system was tested for the highest possible mean local density difference that was expected to be measured. A salt-water-solution buoyant jet was discharged vertically downwards in the observation tank filled with fresh water. An initial density difference of $25.0 \sigma$-units was chosen and, for an initial plume diameter $D=1.25 \mathrm{~cm}$, velocity measurements were taken well into the plume regime (at 45 buoyancy lengthscales $\nmid$ downstream). The resulting beam wobble was minimal and the lenses L1 and L2 shown in figure 3 provided a continuous signal to the photodetectors. The maximum laser-beam excursion was marked on a vertical plane

$\dagger$ The buoyancy lengthscale $l_{M}$ is defined by the initial specific momentum $(\boldsymbol{M})$ and buoyancy (B) fluxes so that $I_{M}=M^{\frac{3}{3}} / B^{\frac{1}{2}}$ (Fischer et al. 1979). 

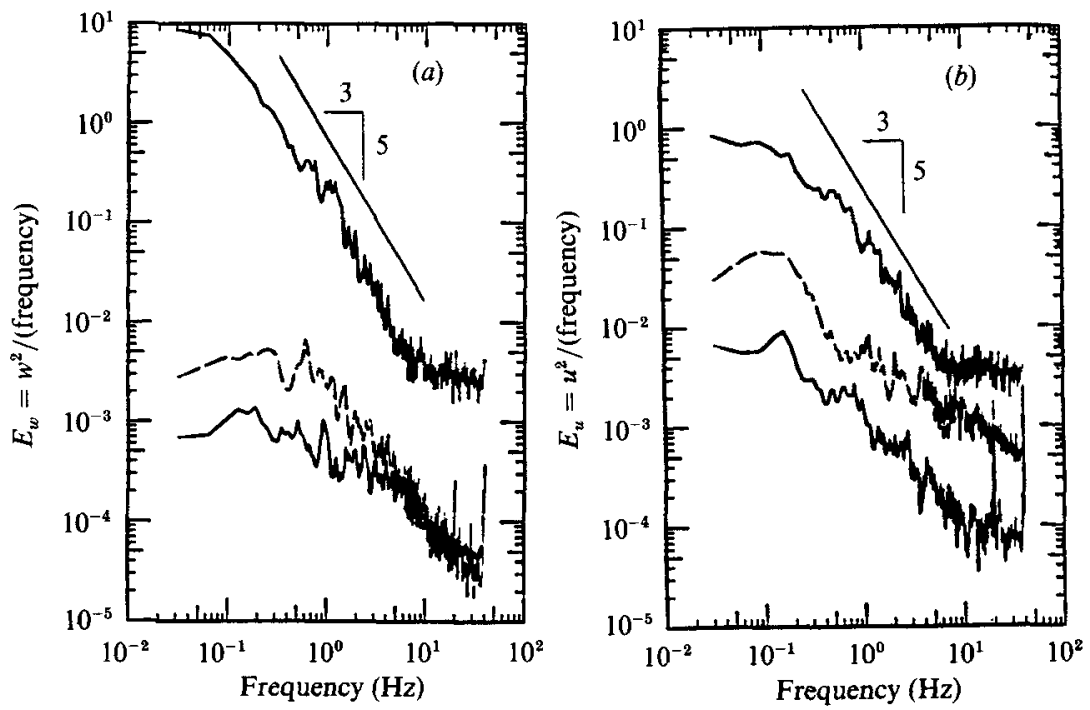

Figure 27. Power spectral estimates $(a)$ for the axial and $(b)$ for the radial velocity components $u$, and $u$ at a plume centreline $\left(z / l_{M}=35.12,\left[(\Delta \rho)_{0} / \rho_{\mathrm{a}}\right] g=27.44 \mathrm{~cm} / \mathrm{s}^{2}\right.$. Noise levels from the processor (lower solid line) and from the changes in the refractive index (dashed line).

outside the tank and the corresponding wobble of $\pm 0.4 \mathrm{~mm}$ determined at the beamcrossing point. A $3.5 \mathrm{~mm}$ thick Plexiglass plate was then introduced into the plume flow field at the beam-crossing point so that the $3 \mathrm{~mm}$ long beam-crossing volume was within the Plexiglass but the beams were left subject to the refractive-index fluctuations in the tank. With the plume running both mean velocities were then measured and found to be zero. The 'turbulence intensities' also did not exceed the values measured in calm fluid. These results clearly imply that the probe volume did not move out of the Plexiglas plate because of the beam wobble nor were fictitious turbulent fluctuations derived. Another test was performed to measure the noise level of the signal due to the changes in the fluid refractive index. A square bottle $3 \times 3$ and $15 \mathrm{~cm}$ high filled with tank water was introduced into the flow at the point containing the beam-crossing. Both longitudinal and transverse velocities were measured with and without the plume running. For calm ambient fluid it was found that inside the bottle $\bar{w}=0 \mathrm{~cm} / \mathrm{s}, \bar{u}=0 \mathrm{~cm} / \mathrm{s},\left(\overline{w^{\prime 2}}\right)^{\frac{1}{2}}=0.069 \mathrm{~cm} / \mathrm{s}$, $\left(\overline{u^{\prime 2}}\right)^{\frac{1}{2}}=0.099 \mathrm{~cm} / \mathrm{s}, \overline{w^{\prime} u^{\prime}}=-0.001 \mathrm{~cm}^{2} / \mathrm{s}^{2}$. With a plume operating with $(\Delta \rho)_{0}=$ $25.0 \mathrm{~g} / \mathrm{l}$ the measured velocities inside the bottle located at $z / l_{M}=52.8$ were $\bar{w}=0 \mathrm{~cm} / \mathrm{s}, \bar{u}=0 \mathrm{~cm} / \mathrm{s},\left(\overline{w^{\prime 2}}\right)^{\frac{1}{2}}=0.099 \mathrm{~cm} / \mathrm{s},\left(\overline{u^{\prime 2}}\right)^{\frac{1}{2}}=0.248 \mathrm{~cm} / \mathrm{s}, \overline{w^{\prime} u^{\prime}}=0.001 \mathrm{~cm}^{2} / \mathrm{s}^{2}$. These measurements indicated that the beam wobble produced maximum noise of almost twice the noise introduced into the system by the various error sources previously discussed. Part of the noise attributed to the beam wobble is probably due to the vibration of the Plexiglas bottle by the plume flow.

In figures $27(a, b)$ the $w$ - and $u$-velocity energy spectra around the plume axis are presented at $z / l_{M}=35.12$ with $\bar{w}=5.48 \mathrm{~cm} / \mathrm{s}, \bar{u}=-0.291 \mathrm{~cm} / \mathrm{s},\left(\overline{w^{\prime 2}}\right)^{\frac{1}{2}}=1.287 \mathrm{~cm} / \mathrm{s}$, $\left(\overline{u^{\prime 2}}\right)^{\frac{1}{2}}=0.695 \mathrm{~cm} / \mathrm{s}$ and $\overline{w^{\prime} u^{\prime}}=0.117(\mathrm{~cm} / \mathrm{s})^{2}$. In these figures the spectra obtained from the signals measured inside the bottle are plotted for comparison. It can be seen that the noise sources make no essential contribution to the energy spectrum. These spectral estimates agree with those of Mizushina et al. (1979), who investigated the pseudoturbulence from the changes in the refractive index in a flow with temperature variation. 


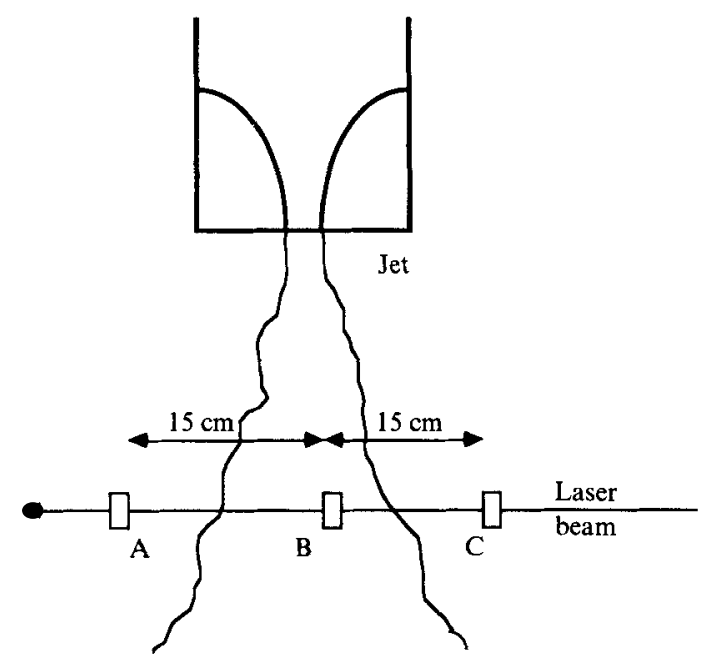

Figure 28. Schematic of the fluorescence attenuation tests performed at the points $A, B$ and $C$.

\section{A.3.2. Calibration and errors of the induced-fluorescence measurement}

The concentration of Rhodamine 6G dye for a laser intensity of $800 \mathrm{~mW}$ is a linear function of the amplified voltage output from the photodetector. It can be written as $c=G+H V$, where $V$ is the digital output to the computer, $G$ and $H$ are calibration constants with $c$ varying from 1 to about 40 p.p.b. The photodiode could not detect Rhodamine $6 \mathrm{G}$ concentrations smaller than 0.4 p.p.b. with an $800 \mathrm{~mW}$ power output from the $2 \mathrm{~W}$ Argon-ion laser. Since the initial jet concentration varied from 100 to 200 p.p.b. solutions were made at various Rhodamine $6 \mathrm{G}$ dye concentrations from 1 to about 40 p.p.b. to represent given levels of dilution. The samples were put in square Plexiglas bottles with cross-section $3 \times 3 \mathrm{~cm}$ and placed sequentially at the laser-beam intersection point so that the photodiode was focused onto the beam section within the known sample concentration. From the known pairs $\left(c_{i}, V_{i}\right)$ of sample concentration and voltage recorded digitally by the computer, a least-square straight-line fit led to the linear equation above. Several different tests were performed in order to evaluate the use, validity and limitations of the technique. The errors resulting from various sources were measured and they will be discussed explicitly in the following paragraphs.

(i) The laser beam is attenuated as it travels through Rhodamine 6G dye solutions. The attenuation is due to the light absorption from the Rhodamine molecules and is proportional to the Rhodamine concentration, and is hence minimized by low Rhodamine 6G concentrations. The attenuation of the laser beam was measured in a test performed as shown in figure 28 . The ambient concentration was measured at point $A$ both with and without a jet of high Rhodamine concentration flowing. The results obtained are

$\begin{array}{ccccl}\nabla & \left(\overline{V^{\prime 2}}\right)^{\frac{1}{2}} & \bar{c} \text { (p.p.b.) } & \left.\overline{\left(c^{\prime 2}\right.}\right)^{\frac{1}{2}} \text { (p.p.b.) } & \\ 1622.0 & 2.77 & 0.757 & 0.246 & \text { (jet flowing), } \\ 1620.7 & 3.27 & 0.873 & 0.290 & \text { (no jet flowing), }\end{array}$

where $V$ is the digital output to the computer, and the difference in concentrations was found to be of the order of 0.1 p.p.b. 


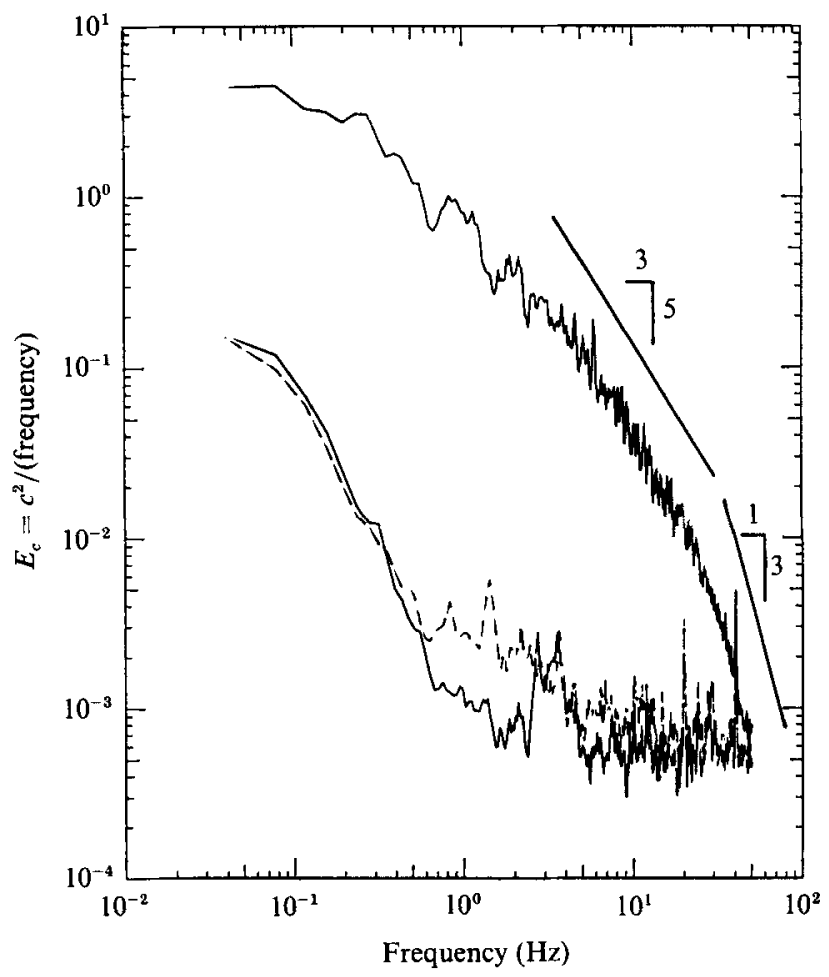

Figure 29. Power-spectral estimate for the concentration at the plume axis (symbols and flow conditions as in figure 27).

(ii) A second source of error is the lateral position of the instrumentation platform. Deflection of the glass panels and slight changes in the beam diameter can produce errors. At points $\mathrm{A}, \mathrm{B}$ and $\mathrm{C}$ shown in figure 28 with $\mathrm{AB}=\mathrm{BC}=15 \mathrm{~cm}$ the concentrations of two Rhodamine $6 \mathrm{G}$ solutions were measured and found to be

$\begin{array}{lccc} & c_{1} \text { (p.p.b.) } & c_{2} \text { (p.p.b.) } & r(\mathrm{~cm}) \\ \text { point A } & 3.783 & 8.743 & -15.0 \\ \text { point B } & 3.728 & 8.468 & 0.00 \\ \text { point C } & 3.599 & 8.457 & +15.0\end{array}$

From the data shown above for small and large concentrations the absolute error with respect to the centreline concentration is at most \pm 0.28 p.p.b. for radial distances $\pm 15 \mathrm{~cm}$. This error is significant close to the jet boundaries, but absolutely insignificant everywhere else. The fact that the concentrations are higher at point $A$ than at point $B$ proves the point that the beam waist changed slightly for various radial distances from the jet axis. The exponential decay of the laser beam intensity through fluid with uniform dye concentration was verified by Hannoun (1986); using his decay exponent for a $10 \mathrm{~cm}$ long fluid volume with uniform concentration of Rhodamine 6G equal to 10 p.p.b. which models a realistic decay path through the plume, the error in the measurement will not exceed $3 \%$ assuming that the induced fluorescence is proportional to the dye concentration for concentrations less than 40 p.p.b.

(iii) A third source of noise is the beam wobble because of the changes in the index of refraction. A square bottle containing a known concentration of Rhodamine was placed in the tank and the concentration was measured with and without the plume 
active. For the flow conditions described in part (v) of $\S$ A.3.1 no significant error was determined. For high density differences the relative error reaches values as large as $8 \%$ of the measured mean concentration. In figure 29 the noise spectrum is compared with the energy spectrum of the concentration fluctuations in the plume flow field.

All the above tests give a reasonable estimate of the errors to be expected. Since simultaneous velocity and concentration measurements were made, it is apparent that the beam wobble could possibly introduce large errors in the turbulent mass transport estimates. In order not to exceed the error estimates made previously in the velocity and concentration measurement, the jet density was chosen carefully to provide appropriate expected local mean densities at the point of measurement. Hence the changes in the refractive index would not introduce an error larger than that previously estimated.

\section{A.3.3. Response; sampling times and rates; spectra of turbulence; experimental procedure}

The response time of the laser-Doppler system with the processor set to count 24-30 periods was $0.001 \mathrm{~s}$. The response time for the laser-induced-fluorescence measurements varied from 0.005 to $0.001 \mathrm{~s}$. Hence the sampling rate was not limited by the response of the instrumentation. At relatively high sampling rates, the sampling time was chosen to be longer than the time necessary to provide constant values of the first three moments of the recorded signal $(100-200 \mathrm{~s})$. The sampling rate varied from $25 \mathrm{~Hz}$ in plumes to $50 \mathrm{~Hz}$ in jets (except for the data acquired in order to examine the spectral properties of the jet). The probe-volume resolution for both velocity and concentration measurements was also a regulatory factor for the choice of the sampling rates. In figures $27(a, b), 29$ and $30(a-c)$ typical energy spectra of $w$, $u$ and $c$ are plotted for jets and plumes. From the spectral-energy distribution it can be seen that the maximum contribution comes from frequencies lower than $10 \mathrm{~Hz}$. The spectral energy drops in the frequency range $0.1-10 \mathrm{~Hz}$ by almost three orders of magnitude. This indicates that the minimum sampling rate should not be less than $20 \mathrm{~Hz}$. Also, for frequencies higher than $10 \mathrm{~Hz}$ the noise contribution becomes very significant. This can be seen in all the figures discussed above. Figures $27(a, b)$ and 29 are plots of the spectral distributions of $w, u$ and $c$ around the plume centreline. Also the noise spectra for measured signals obtained with a calm environment (bottle) are plotted for the two cases with and without changes in the refractive index of the medium around the sample volume. It can be easily observed that the noise contribution is negligible at the low frequencies, and becomes more significant at higher frequencies. The noise level is higher when changes in the refractive index occur (dotted lines in figures), and it is very important near the jet boundary where the signal to noise ratio becomes a minimum.

In a typical experiment to obtain the mean and turbulence characteristics for $w, u$ and $c$ for a jet with a given set of jet initial conditions the following procedures were employed:

(i) $z / l_{M}$ was chosen according to the already selected initial density difference $(\Delta \rho)_{0}$ between the jet discharge and ambient fluid or vice versa.

(ii) Both the observation and mixing tanks were filled with fresh water, and were dechlorinated by adding $\mathrm{Na}_{2} \mathrm{SO}_{3}$. Proper amounts of Rhodamine 6G dye in aqueous solution with known concentration were added and mixed into the tank to provide an ambient fluid concentration close to 1 p.p.b. and the desired jet-fluid concentration. Filtered solute of known high concentration of $\mathrm{NaCl}$ was added 

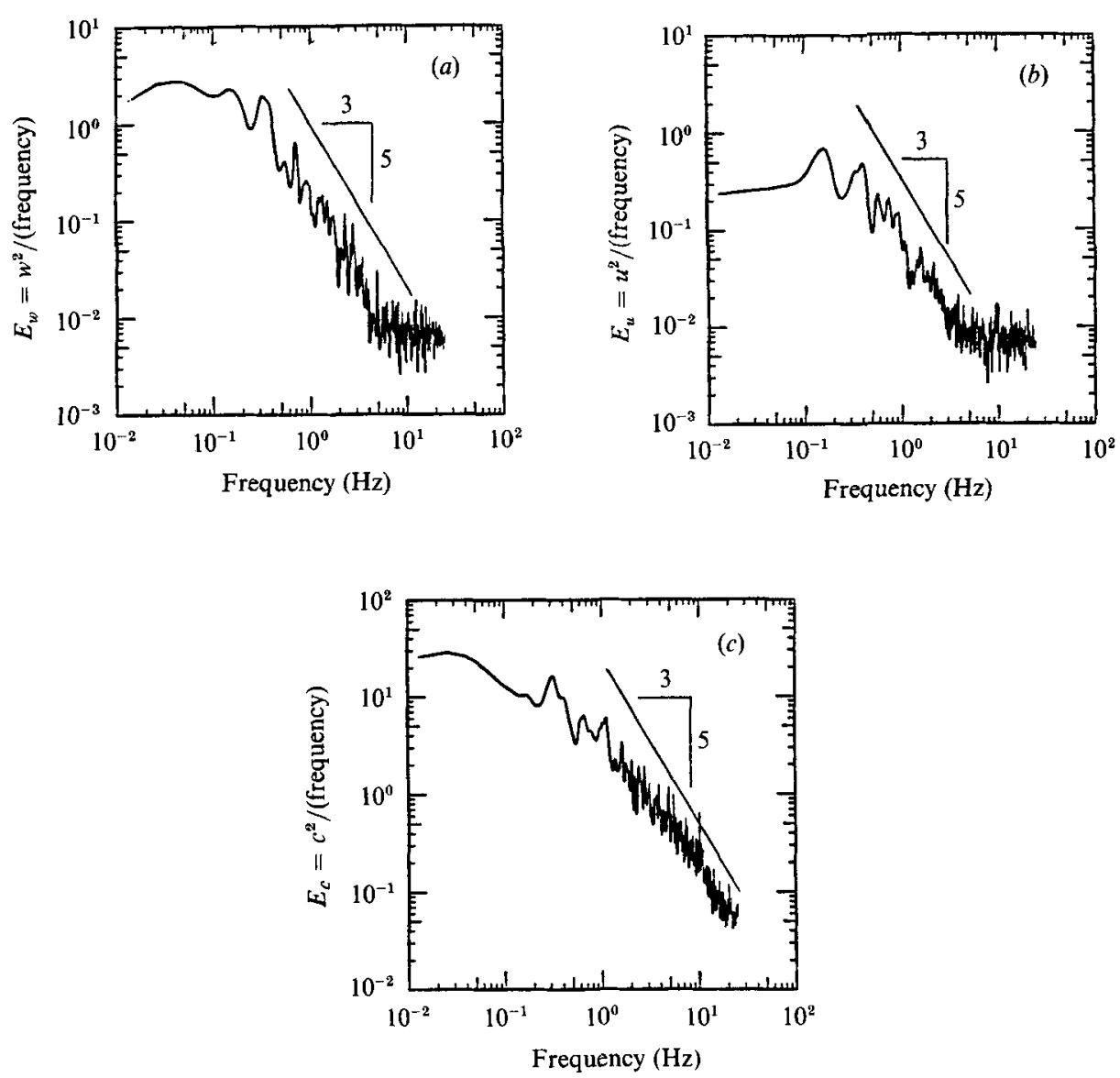

Figure 30. Power spectral estimates of $(a) w,(b), u$ and $(c) c$ at the axis of a jet with $D=1.25 \mathrm{~cm}$, $z / D=38.50, W=29.65 \mathrm{~cm} / \mathrm{s}, R e=3700$ and $C_{0}=98.2$ p.p.b.

accurately so that the jet initial concentration of $\mathrm{NaCl}(\Delta \rho)_{0}$ was known. In this case the jet was denser than the ambient fluid and pointed vertically down.

(iii) The laser-Doppler velocimeter and the induced-fluorescence measuring system were calibrated and the frequency shift between the reference and scattering beams was measured.

(iv) A $10 \mathrm{~s}$ record of the ambient fluid conditions (ambient $w, u$ and $c$ ) was sampled.

(v) After the jet had reached a steady state (for the desired volume flux $Q$ ), data sampling for $w, u$ and $c$ was performed at 10 to 15 different radial distances from the known jet centreline, at the sampling times and rates previously discussed. The velocity and concentration data were transmitted to a PDP 11/60 computer where they were recorded in a binary form for subsequent analysis.

(vi) After the run was completed the laser-induced-fluorescence system was recalibrated.

The binary data were then converted to velocities and concentration using previously derived calibration curves. The converted data were processed properly to provide the desired information about the turbulence both in jets and plumes. Figure $31(a)$ is a representative $20 \mathrm{~s}$ time record of the two velocity components and the 

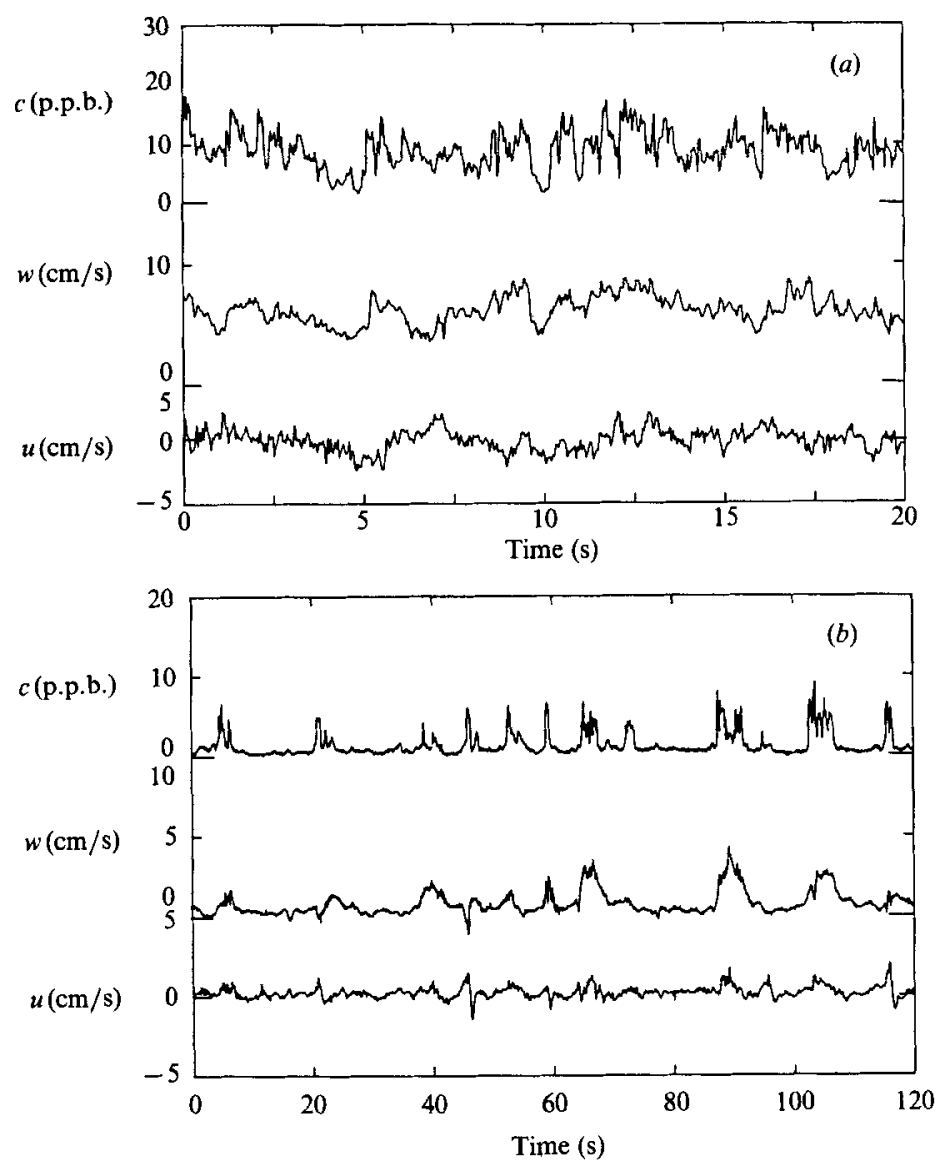

Figure 31. Time record of $w, u$, and $c$ at $z / D=38.90 ;(a)$ on a plume axis $r / z=0$, and $(b)$ at $r / z=0.15$.

concentration measured simultaneously at the same point at the centreline of a plume. Figure $31(b)$ is a $120 \mathrm{~s}$ time history of $u, w$, and $c$ in the highly intermittent regime of the above plume, at a radial distance $r / z=0.15$ from its axis, as discussed in step ( $\mathrm{v}$ ) above. The peak values of the concentration $c$ of the fluorescent dye seem to be in phase with the peak values of the axial velocity component $w$. Hence, the turbulence close to the plume boundary turbulence must transport a significant percentage of the total flux of the fluorescent dye transported by the plume.

\section{A.4. Summary and conclusion}

A non-intrusive laser-light-based experimental technique has been introduced, which enables an acceptably accurate measurement of the turbulent fluxes of momentum and a tracer transported by turbulent buoyant shear flows. The mean and turbulence properties and the fluxes of momentum and a tracer transported by a round buoyant jet are evaluated and discussed extensively in the main body of the paper in both asymptotic jet and plume regions of the flow. The fraction of the tracer flux transported by the turbulence, can be measured accurately by applying the combined LDV-LIF technique, for the following reasons: $(a)$ the measurement of 
both velocity and tracer concentration was performed simultaneously at the same point in the flow; and $(b)$ Doppler anemometry enabled the directional measurement of velocities in the highly intermittent region, around the jet boundary, where reverse flow occurs, and where the turbulence dominates the tracer transport (if compared to that by the mean flow).

The sensitivity in velocimetry $(0.1-0.3 \mathrm{~cm} / \mathrm{s})$ and concentration measurement (0.1 p.p.b.) have allowed the measurement of the turbulence properties at many characteristic jet diameters from the source in the self-similar regime in both jets and plumes, where intrusive probe-based techniques have failed. High initial jet concentrations of Rhodamine $6 \mathrm{G}$ dye (250 p.p.b.), which allow accurate measurement very far from the source, did not affect the initial buoyancy input at the jet source, contrary to the use of heat or salinity as tracers for the concentration measurement. However, the aspect ratio of the tank cross-section versus the jet source diameter which varied from 75 (plumes) to 155 (jets), has limited the measurement to 110 jet and 80 plume diameters from the source. Measurements at axial distances greater than 100 characteristic diameters from the source were greatly affected by recirculation of the tank fluid.

Although the optical measuring technique applied, by its nature, does not interfere with the flow as do most of the intrusive probe-based methods, it can be greatly affected by the changes in the refractive index in the flow, resulting in pseudoturbulence and mobility of the point of the Eulerian measurement, because of beam wobble. In the present study beam wobble was controlled by performing measurements in the flow region where $\mathrm{NaCl}$ concentration was very low, rather than by matching the refractive indices of the jet and ambient fluid.

In spite of its advantages, the combined LDV-LIF technique is limited in its application because of the combined disadvantages of LDV and LIF. For example, the size of the tank was limited to $1.20 \mathrm{~m}$ because of the small coherence length of the $7.5 \mathrm{~mW}$ laser used in the LDV, and because of the decay of the fluorescent light from the $800 \mathrm{~mW}$ laser beam being inversely proportional to the distance from the LIF receiving optics. Furthermore, as has been noted earlier, the LIF employed in the buoyant jet measurement overestimated the time-averaged mean concentration of fluorescent tracer. The cause of this error appears to be in excess light collected by the receiving optics for the LIF from scattering centres within the flow field. It seems likely also that this overestimation may have been contributed to by adsorption of fluorescent dye onto the small organic scattering particles suspended in the flow, necessary for operation of the laser-Doppler system but not present in the concentration calibration solutions. Future investigators using these techniques should carefully evaluate the above sources of possible error.

\section{REFERENCES}

Abbiss, J. B., Bradbury, L. J. S. \& Wright, M. P. 1975 Measurements on an axisymmetric jet using a photon correlator. In Proc. LDA Symp. Copenhagen, pp. 319-335.

Aвraham, G. 1960 Jet diffusion in liquid of greater density. J. Hydraul. Div. ASCE, 86, 1-13.

Albertson, M. L., Dai, Y. B., Jensen, R. A. \& Rouse, H. 1950 Diffusion of submerged jets. Trans. ASCE 115, 639-664.

Antonia, R. A., Chambers, A. J. \& Hussatn, A. K. M. F. 1980 Errors in simultaneous measurements of temperature and velocity in the outer part of a heated jet. Phys. Fluids 23, 871-874.

Antonia, R. A., Prabhu, A. \& Stephenson, S. E. 1975 Conditionally sampled measurements in a heated turbulent jet. $J$. Fluid Mech. 72, 455480. 
Becker, H. A., Hottel, H. C. \& Williams, G. C. 1967 The nozzle-fluid concentration field of the round turbulent free jet. $J$. Fluid Mech. 30, 285-303.

Birch, A. D., Brown, D. R., Dodson, M. G. \& Thomas, J. R. 1978 The turbulent concentration field of a methane jet. J. Fluid Mech. 88, 431-449.

Chevray, R. \& Tutu N. K. 1978 Intermittency and preferential transport of heat in a round jet. J. Fluid Mech. 88, 133-160.

Corrsin, S. 1943 Investigation of flow in an axially symmetric heated jet of air. NACA Wartime Rep. W-94.

Corrsin, S. \& Uberor, M. S. 1949 Further experiments on the flow and heat transfer in a heated turbulent air jet. NACA Rep. 998.

Drain, L. E. 1980 The Laser Doppler Technique. Wiley.

Drexhage, K. H. 1976 Structure and properties of laser dyes. From Topics in Appl. Physics, 1, Springer.

Durst, F., Melling, A. \& Whitelaw, J. H. 1976 Principles and Practice of Laser Doppler Anemometry. Academic.

Fischer, H. B., List, E. J., Koh, R. C. Y., Imberger, J. \& Brooks, N. H. 1979 Mixing in Inland and Coastal Waters. Academic.

Forstall, W. \& GayloRd, E. W. 1955 Momentum and mass transfer in a submerged water jet Trans. ASME E.: J. Appl. Mech. 22, 161-164.

GaRtrelL, G. 1978 A signal processor for a laser-Doppler velocimeter. Tech. Memo. 78-5. W. M. Keck Laboratory of Hydraulics and Water Resources, California Inst. of Technology, Pasadena, California.

Gartrkll, G. 1979 Studies on the mixing in a density-stratified shear flow. Rep. KH-R-39. W. M. Keck Laboratory of Hydraulics and Water Resources, California Institute of Technology, Pasadena, California.

George, W. K., Alpert, R. L. \& Tamanini, F. 1977 Turbulence measurements in an axisymmetric buoyant plume. Intl J. Heat Mass Transfer 20, 1145-1154.

GiBson, M. M. 1963 Spectra of turbulence in a round jet. J. Fluid Mech. 15, 161-173.

Grandmaison, E. W., Rathgeber, D. E. \& Becker, H. A. 1977 Some characteristics of concentration fluctuations in free turbulent jets. In Preprints, Symposium on Turbulent Shear Flows, Penn. State Univ., pp. 15.21-15-29.

Hannoun, I. A. 1986 Turbulent mixing in stably stratified fluid subjected to zero mean shear. Ph.D. thesis, W. M. Keck Laboratory of Hydraulies and Water Resources, California Inst. of Technology, Pasadena, California.

Hinze, J. O. \& van DeR HeGge ZiJen, B. G. 1949 Transfer of heat and matter in the turbulent mixing zone of an axially symmetric jet. Appl. Sci. Res. A1, 435-461.

Hussain, A. K. M. F. \& ClaRK, A. R. 1981 On the coherent structure of the axisymmetric mixing layer: a flow visualization study. J. Fluid Mech. 104, 263-294.

Johnson, B. V. \& BENNETT, J. C. 1981 Mass and momentum turbulent transport experiments with confined coaxial jets. NASA Rep. CR-165574.

KISER, K. M. 1963 Material and momentum transport in axisymmetric turbulent jets of water. AIChEJ. 9, 386-390.

Kotsovinos, N. E. 1975 A study of the entrainment and turbulence in a plane jet. Rep. KH-R-32. W. M. Keck Laboratory of Hydraulics and Water Resources, California Inst. of Technology, Pasadena, California.

Kotsovinos, N. E. 1976 A note on the spreading rate and virtual origin of a plane turbulent jet. J. Fluid Mech. 77, 305-311.

Kotsovinos, N. E. 1977 Plane turbulent buoyant jets. Part 2. Turbulence structure. J. Fluid Mech. 81, 45-62.

Kotsovinos, N. E. 1978 A note on the conservation of the axial momentum of a turbulent jet. J. Fluid Mech. 87, 55-63.

Kotsovinos, N. E. 1985 Temperature measurements in a turbulent round plume. Intl J. Heat Mass Transfer 28 771-777.

Kotsovinos, N. E. \& List, E. J. 1976 Turbulent buoyant jets. In Proc. 1976 ICHMT Seminar on Turbulent Buoyant Convection, pp. 349-359. Hemisphere. 
Kotsovinos, N. E. \& List, E. J. 1977 Plane turbulent buoyant jets. Part 1. Integral properties. J. Fluid Mech. 81, 25-44.

LaU, J. C., Morris, P. J. \& Fisher, M. J. 1979 Measurements in subsonic and supersonic free jets using a laser velocimeter. $J$. Fluid Mech., 93, 1-27.

List, E. J. 1982 a Turbulent jets and plumes. Ann. Rev. Fluid Mech. 14, 189-212.

List, E. J. $1982 b$ Mechanics of turbulent buoyant jets and plumes. In Turbulent Buoyant Jets and Plumes, pp. 1-68. Pergamon.

List, E. J. \& Imberger. J. 1973 Turbulent entrainment in buoyant jets. J. Hydraul Div. ASCE $99,1461-1474$.

McDovgali, T. J. 1979. On the elimination of the refractive index variations in turbulent density-stratified liquid flows. J. Fluid Mech 93, 83-96.

Mizushina, T., Ogino, F., Veda, H. \& Komori, S. 1979 Application of laser-Doppler velocimetry to turbulence measurement in non-isothermal flow. Proc. R. Soc. Lond. A366, 63-79.

Morton, B. R., Taylor, G. I. \& Turner, J.S. 1956 Turbulent gravitational convection from maintained instantaneous sources. Proc. R. Soc. Lond. A234, 1-23.

Muellenhof, W. P., Soldate, A. M., Baumgartner, D. J., Schuldt, M. D., Davis, L. R. \& FrIck, W. E. 1985 Initial mixing characteristics of municipal ocean discharges. Vol. 1, Procedures and applications. EPA/600/3-85/073a, Nov. 1985, $90 \mathrm{pp}$.

Nakagome, H. \& Hirata, M. 1976 The structure of turbulent diffusion in an axisymmetric thermal plume. Proc 19\%6 ICHMT Seminar on Turbulent Buoyant Convection, pp. 361-372. Hemisphere.

OwEN, F. K. 1976 Simultaneous laser measurements of instantaneous velocity and concentration in turbulent mixing flows. AGARD Conf. Proc. 193, 27-1-27-7.

Papanicolaod, P. N. 1984 Mass and momentum transport in a turbulent buoyant vertical axisymmetric jet. Rep. KH-R-46, May 1984. W. M. Keck Laboratory of Hydraulics and Water Resources, California Inst. of 'Technology, Pasadena, California.

Papanicolaou, P. N. \& List, E. J. 1984 Turbulent mass and momentum transport in round vertical turbulent buoyant jets. Second Intl Symp. on Applications of Laser-Doppler Anemometry to Fluid Mechanics, Lisbon, Portugal.

PAPANicolaou, P. N. \& LIST, E. J. 1987 Statistical and spectral properties of tracer concentration in round buoyant jets. Inll J. Heat and Mass Transfer 30, 2059-2071.

Ramaprian, B. R. \& Chandrasekhara, M. S. 1983 Study of vertical plane turbulent jets and plumes. IIHR Rep. 257, Iowa Inst. of Hydraulic Research.

Ricou, F. P. \& Spalding, D. B. 1960 Measurements of entrainment by axisymmetrical turbulent jets. J. Fluid Mech. 11, 21-32.

Rosenweig, R. E., Hottel, H. C. \& Williams, G. C. 1961 Smoke scattered light measurement of turbulent concentration fluctuations. Chem. Engng Sci. 15, 111-129.

Rosler, R. S. \& BAnkofF, S. G. 1963 Large scale turbulence characteristics of a submerged water jet. AIChEJ. 9, 672-676.

Rouse, H., YiH, C. S. \& Humphreys, H. W. 1952 Gravitational convection from a boundary source. Tellus 4, 201-210.

Sforza, P. M. \& Mons, R. F. 1978 Mass, momentum and energy transport in turbulent free jets. Intl J. Heat Mass Transfer 21, 371-384.

TAYLOR, G. I. 1958 Flow induced by jets. J. Aero. Sci. 25, 464-465.

Watrasiewicz, B. M. \& RudD, M. J. 1976 Laser-Doppler Measurements. Butterworth.

Wilson, R. A. M. \& Danckwekts, P. V. 1964 Studies in turbulent mixing II : A hot air jet. Chem. Engng. Sci. 19, 885-895.

Wygnanski, I. \& Fiedler, H. 1969 Some measurements in the self-preserving jet. J. Fluid Mech. $38,577-612$.

ZIMIN, V. D. \& FRIK, P. G. 1977 Averaged temperature fields in asymmetrical turbulent streams over localized heat sources. izv. Akad. Nauk. SSSR, Mekh. Zhidi. Gaza 2, 199-203. 